\title{
High-precision stellar abundances of the elements Methods and applications
}

\author{
Poul Erik Nissen • Bengt Gustafsson
}

Received: date / Accepted: date

\begin{abstract}
Efficient spectrographs at large telescopes have made it possible to obtain high-resolution spectra of stars with high signal-to-noise ratio and advances in model atmosphere analyses have enabled estimates of high-precision differential abundances of the elements from these spectra, i.e. with errors in the range 0.01 0.03 dex for $\mathrm{F}, \mathrm{G}$, and $\mathrm{K}$ stars.

Methods to determine such high-precision abundances together with precise values of effective temperatures and surface gravities from equivalent widths of spectral lines or by spectrum synthesis techniques are outlined, and effects on abundance determinations from using a 3D non-LTE analysis instead of a classical 1D LTE analysis are considered.

The determination of high-precision stellar abundances of the elements have led to the discovery of unexpected phenomena and relations with important bearings on the astrophysics of galaxies, stars, and planets, i.e. $i$ ) Existence of discrete stellar populations within each of the main Galactic components (disk, halo, and bulge) providing new constraints on models for the formation of the Milky Way. $i$ i) Differences in the relation between abundances and elemental condensation temperature for the Sun and solar twins suggesting dust-cleansing effects in proto-planetary disks and/or engulfment of planets by stars; iii) Differences in chemical composition between binary star components and between members of open or globular clusters showing that star- and cluster-formation processes are more complicated than previously thought; $i v$ ) Tight relations between some abundance ratios and age for solar-like stars providing new constraints on nucleosynthesis and Galactic chemical evolution models as well as the composition of terrestrial exoplanets.
\end{abstract}

P. E. Nissen

Stellar Astrophysics Centre, Department of Physics and Astronomy, Aarhus University, Ny Munkegade 120, 8000 Aarhus C, Denmark.

E-mail: pen@phys.au.dk

B. Gustafsson

Department of Physics and Astronomy, Uppsala University, Box515, 75120 Uppsala and Nordita, Stockholm, Sweden.

E-mail: Bengt.Gustafsson@physics.uu.se 
We conclude that if stellar abundances with precisions of $0.01-0.03$ dex can be achieved in studies of more distant stars and stars on the giant and supergiant branches, many more interesting future applications, of great relevance to stellar and galaxy evolution, are probable. Hence, in planning abundance surveys, it is important to carefully balance the need for large samples of stars against the spectral resolution and signal-to-noise ratio needed to obtain high-precision abundances. Furthermore, it is an advantage to work differentially on stars with similar atmospheric parameters, because then a simple 1D LTE analysis of stellar spectra may be sufficient. However, when determining high-precision absolute abundances or differential abundance between stars having more widely different parameters, e.g. metal-poor stars compared to the Sun or giants to dwarfs, then 3D non-LTE effects must be taken into account.

Keywords Techniques: spectroscopic - Stars: abundances - Stars: fundamental parameters - - Planet-star interactions - Galaxy: disk -Galaxy: halo - Galaxy: bulge - Galaxy: evolution

\section{Introduction}

The chemical analysis of stars by stellar spectroscopy provides basic data of key significance for a broad range of areas in contemporary astronomy and astrophysics, extending from the study of stellar evolution, of the formation of planetary systems, of Galactic and galaxy evolution, to cosmology. The basis of the analysis was founded already in the early 19th century on the discovery of the dark Fraunhofer lines in the solar spectrum, the line identifications with terrestrial elements some decades later, and the first observations of stellar spectra in the second half of the century. However, it was not until the progress in atomic and molecular physics during the first half of the 20th century that quantitative chemical analysis was possible and gave important results. In recent decades, the methods of analysis have been developed impressively, due to progress in three areas: One is the advent of telescopes with large light collecting area, comparatively effective spectrometers and sensitive detectors with linear response to illumination. Earlier, the low efficiency of instruments and the non-linear properties of photographic emulsions plagued the analyses - now it is possible to obtain spectra for hundreds of thousands of stars of a quality which 50 years ago were only available for the Sun and a handful of bright stars. Very important are also the advances in laboratory measurements and calculations of accurate atomic and molecular data, in particular line identifications, oscillator strengths and other relevant cross sections. Previous data, e.g. for oscillator strengths for lines of the most important elements, could suffer from systematic errors of a full order of magnitude, if available at all. The progress in the third respect, the theoretical modelling of the spectrum-emitting regions, the stellar atmospheres, and of their predicted spectra, is as impressive. It has now become possible to construct models that are as physically consistent as can be reasonably required: to consider the effects of the spectral lines on the structure of the atmospheres in full detail, to replace the previous assumptions of plane-parallel stratification and simplified models of the convective energy transport (primarily the Mixing-Length "Theory", MLT) with a full solution in 3D of the hydrodynamic equations as well as the radiative energy transfer, and in the spectrum calculation to adopt the realistic velocity fields 
from the hydrodynamic simulations and also abandon the previously made Local Thermodynamic-Equilibrium (LTE) assumption - that is to allow for all the nonlocal radiative effects on the excitation and de-excitation of each atomic level, and on the ionisation and recombination of each ionisation stage, of significance (in the literature denoted "non-LTE"). In practice, these theoretical advances have led to improvements which in many cases are decisive, not the least for the analysis of metal-poor stars where the thermal inhomogeneities and the non-local radiative fields may strongly affect the visible stellar spectrum. These improvements are well described by Asplund (2005).

Since long ago, the errors in spectroscopic stellar-abundance determinations have been disputed, but often systematically underestimated. The presence of systematic errors in observations, interpretations and models have been hard to take into account fully, since there has been an absence of reliable comparisons for error calibration; standard spectra and analyses have also suffered from error sources similar to those of the programme stars like saturation effects, misidentified or unidentified spectral lines, systematic errors in physical line-data, and severe departures from the model-assumptions of LTE and MLT. In this situation, the error estimates in standard abundance analyses have most often been intrinsic, based on the scatter in results for different individual spectral lines, at best also representing different species (ions, molecules) in which the element in question is present. Other sources of error which are also usually considered in these estimates are the errors in fundamental parameters such as effective temperature $T_{\text {eff }}$ and surface gravity $g=G M / R^{2}$ (where $M$ is stellar mass, $R$ radius and $G$ is Newton's constant of gravity). Sometimes also the estimated uncertainties in fudge parameters, in particular the microturbulence parameter $\xi_{\text {turb }}$ that is needed when partially saturated spectral lines are used in standard 1D model analyses, are included in the error analyses. Also, in the more refined 3D analyses in non-LTE, a number of errors in physically well-defined quantities such as collision cross sections and dissociation energies may be important and have to be included in the discussion of abundance errors.

Just as the spectral abundance analyses have improved considerably during recent years, one must expect that the error analyses have improved, not the least due to the considerably more consistent models as such, which, even if they are yet not in regular use, serve as comparison when judging what errors are to be expected in standard analyses. During the last decade, a number of analyses have been published where such a detailed discussion has demonstrated errors smaller than 0.04 dex in the logarithm of the abundance. In all cases, these are errors in relative abundances, i.e. as compared with a standard star, such as the Sun (and relative to hydrogen as the dominating element). The absolute determination of abundances is more difficult. In the global study of solar abundances by Asplund et al (2009) typical abundance errors range from 0.04 to $0.10 \mathrm{dex}$. The reason why the relative errors in stellar abundances may be smaller than those solar absolute ones is that some systematic errors, notably those related to oscillator strengths and model uncertainties, may be assumed to cancel efficiently if the star is physically not too different from the standard star. This approach is basically built on the finding that stellar spectra may indeed be characterized by a small number of stellar fundamental parameters. The degree to which it may be applied will be further discussed below. 
Before proceeding into a more technical discussion of the reliability of claimed high-precision abundance determinations, it is of interest to consider what use such high precisions (errors less than $0.04 \mathrm{dex}$ ) could have. When summarizing the discussion of an ESO stellar-abundance workshop in 1980, celebrating the new ESO CAT/CES spectrometer with its linear Reticon detector arrays which brought us several important steps forward in these analyses, Bernard Pagel made the interesting distinction between two categories of stellar spectroscopists: the "broad sweepers" and the "ultimate refiners". The broad sweepers were primarily interested in global Galactic evolution problems, and were indeed often satisfied with an accuracy on the order of 0.2 dex in their abundance results, while the ultimate refiners wanted more accurate data, often driven by stellar physics considerations. This situation is now changed. A number of discoveries and ideas have increased the interest in high-precision abundances, even among scientists that are not primarily interested in stars in themselves. One could, in principle, see this interest as a result of an often-made experience in natural sciences: when the accuracy of measurements is increased significantly, new important structures in nature are disclosed. Among these proposed or discovered new structures are the following:

1. The possibility to distinguish different stellar components, or sub-populations, in the Galaxy by finding certain common special abundance profiles among stars ("chemical tagging", Freeman and Bland-Hawthorn 2002) and from that infer and map the early history of the Galaxy, with in-falling and merging minor systems. This idea has got a wider application, also for studies of the evolution of the Galactic thin and thick disks and its bulge with some interesting results. The field is further stimulated by systematic combination of data from the astrometric satellite Gaia with high-resolution spectroscopy.

2. The systematic comparison of solar twins 1 with the Sun which has unexpectedly proven that the Sun is somewhat poor in refractory elements as compared with most twins in the solar neighbourhood (Meléndez et al 2009). This finding, where the Sun departs in composition as a function of the dustcondensation temperature of the element in question, has opened up the possibility to study the conditions in the environment where the Sun was once formed, and a search for Solar siblings in the Galaxy.

3. The study of the possibly different chemical composition of the components of binaries. Laws and Gonzalez (2001) and Ramírez et al (2011) found that the secondary star of $16 \mathrm{Cyg}$, known to host a giant planet, is slightly metal-poor relative to the primary, and similar effects have later been found for other binaries (e.g. Saffe et al 2017; Oh et al 2018). It seems that these abundance differences give information on the early evolution of these systems, including their planets.

4. The study of different chemical composition among stars in clusters. For long, differences, including anti-correlations, between the abundances of the light elements, like $\mathrm{C}, \mathrm{O}, \mathrm{Na}, \mathrm{Mg}$ and $\mathrm{Al}$ have been noticed for globular clusters (Cohen 1978; Peterson 1980; Kraft et al 1993; Gratton et al 2001b), and interpreted in terms of self-enrichment by matter from early generations of stars in the forming cluster. Several details in such a scenario are, however, not

\footnotetext{
1 A solar twin is usually defined as a star having atmospheric parameters agreeing with those of the Sun within $\pm 100 \mathrm{~K}$ in effective temperature and \pm 0.10 dex in surface gravity and metallicity.
} 
understood and depend on further detailed studies. Similarly, the relatively small effects of differential diffusion of heavy elements in the stars were traced by Korn et al (2007) for the cluster NGC 6397 and such studies have been pursued to other more metal-rich clusters (e.g. Önehag et al 2014). For open clusters several studies have been devoted to the search for abundance differences among cluster stars with negative or non-significant results. A first sign of chemical inhomogeneities at the $0.02-0.04$ dex level among the solar-type stars in the Hyades and Pleiades clusters was, however, reported by Liu et al (2016b) and Spina et al (2018a).

5. Chemical abundances for the study of nucleosynthesis and stellar evolution. In general, the study of the composition of stars of different ages and at different location in the Galaxy (and other galaxies) lead to information on mixing processes in the interstellar medium as well as in the stars when nuclear reactions are brought to the stellar surface. The very accurate measurement of these abundances are sometimes of key significance for the understanding of the underlying processes, see e.g. review by Nomoto et al (2013).

6. The search for abundance signatures in planetary hosts of different kinds. Early on it was realized that stars of solar type with known massive planets were comparatively metal-rich (Gonzalez et al 2001; Santos et al 2001) but a similar effect for different types of stars and planetary systems is disputed. Other effects of planets, on the abundances of lithium and other elements in the planetary hosts are studied with ambiguous results. Conversely, abundances in stars set constraints on the composition of proto-planetary disks and hence on the composition of planets.

These examples of discoveries when high-precision abundance analyses are applied also suggest new possibilities for future important work on other types of stars or on more distant stars for which very accurate analysis may be attainable within a near future. However, to achieve an accuracy of 0.03 dex or better, improved understanding of the structure and dynamics of the atmospheres of the corresponding stars will be needed together with sufficiently complete atomic and molecular data, as well as adequate observing time at larger telescopes in order to secure sufficiently high $\mathrm{S} / \mathrm{N}$ and spectral resolution. Then, the chemical composition of red giant stars, AGB stars and even supergiants may be explored in detail and used in the study of more distant regions, in the Galaxy and in external galaxies.

\section{Methods}

As a first step towards high-precision abundances of elements in stars, one has to obtain stellar spectra with high resolution and high signal-to-noise. Spectral lines are Doppler broadened due to thermal and turbulent motions of absorbing atoms in stellar atmospheres, which for late-type stars corresponds to a profile with a FWHM of $\sim 6 \mathrm{~km} \mathrm{~s}^{-1}$. Hence, in order to avoid a significant additional instrumental broadening of spectral lines, one needs a spectrograph with a resolution, $R=\lambda / \Delta \lambda_{\text {instr }} \gtrsim 50000$, where $\Delta \lambda_{\text {instr }}$ is the FWHM of the instrumental profile. This makes it easier to disentangle blended lines and define a reliable continuum. However, lower resolution may also be used to determine high-precision abun- 
dances when using spectrum synthesis techniques, if an accurate and sufficiently complete list of lines is available for the spectral region(s) studied.

2.1 Equivalent width measurements and abundance errors

In most high-precision studies, abundances have been derived from equivalent widths (EWs) measured by fitting Gaussian profiles to the spectral lines or by direct integration, i.e.

$$
E W=\sum_{i=1}^{n_{p i x}} \frac{F_{c}-F\left(\lambda_{i}\right)}{F_{c}} \delta \lambda_{p i x}
$$

where $F_{c}$ is the continuum flux, $F\left(\lambda_{i}\right)$ the flux in the line at a particular wavelength point $\lambda_{i}, \delta \lambda_{p i x}$ the spectral pixel size, and $n_{p i x}$ the number of pixels aross the line. For a given value of $F_{c}$, the statistical error of the EW is then

$$
\sigma(E W) \simeq \sqrt{n_{\text {pix }}}(S / N)^{-1} \delta \lambda_{\text {pix }}
$$

where $S / N$ is the signal-to-noise ratio per spectral pixel assumed to be approximately constant across the line.

With three pixels per spectral resolution element $\left(\Delta \lambda_{\text {instr }}=3 \delta \lambda_{\text {pix }}\right)$ as in many high-resolution echelle spectrographs, we get $\delta \lambda_{\text {pix }}=0.04 \AA$ at $\lambda=6000 \AA$ for a resolution of $R=50000$. The flux in the line is typically integrated over a width of $0.4 \AA$ corresponding to $n_{\text {pix }} \simeq 10$. Hence, a S/N of 200 is sufficient to measure the EW with a statistical error of $0.6 \mathrm{~m} \AA$, which for a line with $E W \simeq$ $25 \mathrm{~m} \AA$ corresponds to a relative error of $2.4 \%$. As the abundance derived from such a weak line (on the linear part of the curve of growth) is nearly proportional to the equivalent width, the corresponding error in logarithmic abundance is $\sim 0.01$ dex.

Uncertainties in the continuum setting may introduce larger errors. A shift of the continuum by $0.5 \%$ changes the EW by $2 \mathrm{~m} \AA$ in the example given above, i.e. more than three times the statistical error given by Eq. (2). The continuum effect on differential abundances can, however, be kept below 0.01 dex, if EWs are measured relative to the same continuum windows and a set of spectral lines having similar strengths for all programme stars are used. If, on the other hand, a wide range in abundances is to be covered, large differences in line strength are present and it may not be possible to use the same set of spectral lines in all stars, which means that the abundances derived are sensitive to the continuum setting and are also affected by errors in the $g f$-values. For this reason, trends of abundance ratios as a function of $[\mathrm{Fe} / \mathrm{H}]$ are not as precise as differential abundances at a given metallicity.

As shown by Bedell et al (2014), it is important to observe all program stars with the same spectrograph at a fixed resolution, if differential abundances with a precision of $\sim 0.01$ dex are to be obtained. This requirement raises special problems if the Sun is used as a standard, as is often the case, because the solar flux spectrum cannot be observed in direct sunlight in the same way as the stellar spectra. One may, however, use reflected sunlight from asteroids to obtain a good proxy of the solar flux spectrum. Bedell et al (2014) show that abundances derived from $S / N \sim 700$ spectra of Vesta, Iris, and Ceres observed with the same spectrograph agree with a rms scatter of 0.006 dex. A similar good agreement between spectra 

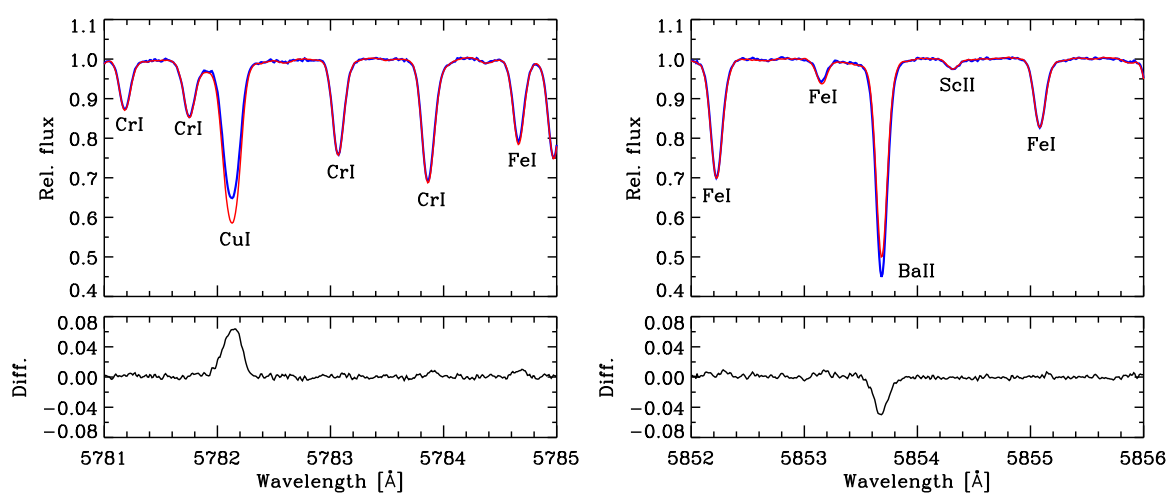

Fig. 1 Solar flux ESO 3.6 m HARPS spectrum (red line) in comparison with the HARPS spectrum of HD 96116 (blue line) near the $\mathrm{Cu}$ I line at $5782.12 \AA$ (left panel) and the Ba II line at $5853.70 \AA$ (right panel). The lower panels show the difference (HD 96116 - Sun) between the two spectra. Based on spectra from Nissen (2016).

of Vesta, Victoria and the Jupiter moon Europa observed with the HERMES spectrograph at the $1.2 \mathrm{~m}$ Mercator telescope was obtained by Beck et al (2016). Furthermore, they found that the normalized spectrum of Europa $(S / N \sim 450)$ agrees with the revised Kitt Peak Solar Flux Atlas of Kurucz (2005) (degraded to the resolution of HERMES, $R=85000$ ) with a rms deviation of $0.25 \%$ over the spectral range $4000-8000 \AA$ (excluding regions with strong telluric lines). This suggests that such reflected sunlight spectra are adequate for determining differential stellar abundances with respect to the Sun at a precision better than 0.01 dex, but further investigations should be made, given that the Sun is found to have an unusual ratio between volatile and refractory elements compared to solar twins as discussed in Sect. 4 .

As an illustration of the differential way of measuring equivalent widths, Fig.1 shows the ESO $3.6 \mathrm{~m}$ HARPS spectrum $(R=115000, S / N \simeq 600)$ of the young solar twin star, HD 96116, in comparison with the solar flux HARPS spectrum $(S / N \sim 1200)$ observed in reflected sunlight from the asteroid Vesta. As seen the $\mathrm{Cr}$ I and Fe I lines have nearly the same strengths in the two stars, whereas the Ba II line is stronger for HD $96116(\Delta E W=7.7 \mathrm{~m} \AA)$ and the $\mathrm{Cu}$ I line is weaker $(\Delta E W=-13.7 \mathrm{~mA})$. Taking into account the small differences in atmospheric parameters between HD 96116 and the Sun, Nissen (2016) has used the differences in EWs to derive $[\mathrm{Ba} / \mathrm{Fe}]^{2}=0.190$ and $[\mathrm{Cu} / \mathrm{Fe}]=-0.098$ for HD 96116 in a study of trends of abundance ratios as a function of stellar age.

In most high-precision abundance studies (e.g. Meléndez et al 2009; Ramírez et al 2014; Tucci Maia et al 2014; Nissen 2015; Spina et al 2016b), equivalent widths have been measured by interactive fitting of weak to medium-strong lines $(E W \lesssim 80 \mathrm{~m} \AA$ in the visual spectral region) by Gaussian profiles using the IRAF splot task. This has the advantage that problems of line blending can be directly seen and eventually solved by using the deblending routine in splot. For very weak lines and lines with asymmetric or box-like profiles due to hyper-fine-structure (HFS), direct

${ }^{2}$ For two elements, $\mathrm{A}$ and $\mathrm{B}$, with number densities $N_{\mathrm{A}}$ and $N_{\mathrm{B}},[\mathrm{A} / \mathrm{B}] \equiv \log \left(N_{\mathrm{A}} / N_{\mathrm{B}}\right)_{\text {star }}-$ $\log \left(N_{\mathrm{A}} / N_{\mathrm{B}}\right)_{\text {Sun }}$. 
integration of the line is to be preferred. Strong lines with damping wings should be measured by Voigt profile fitting, but the resulting EWs are sensitive to weak blends in the wings and to the continuum setting, so such lines should be avoided if possible.

Interactive measurements of EWs are, however, time-consuming and unsuitable for large surveys in which millions of lines have to be measured 3 . Programs for automated measurement of EWs have therefore been developed, e.g. DAOSPEC (Stetson and Pancino 2008), iSpec (Blanco-Cuaresma et al 2014), and ARES v2 (Sousa et al 2015). and extensively applied for stellar abundance studies. It would be interesting to see how well high precision abundances based on EWs measured by such programs agree with abundances based on interactive measurements of EWs.

\subsection{Stellar parameters}

In order to derive high-precision stellar abundances, the effective temperature, $T_{\text {eff }}$, and surface gravity, $g$, of a star should also be known with high precision. For latetype stars, abundances derived from equivalent widths of low-excitation neutral metal-lines change by up to 0.1 dex if $T_{\text {eff }}$ of the model atmosphere used to analyse the lines is changed by $100 \mathrm{~K}$, and abundances derived from ionized metal-lines may be subject to a similar change if $\log g$ is changed by 0.3 dex. Hence, the stellar parameters should be determined with precisions of $\sigma\left(T_{\text {eff }}\right) \simeq 10 \mathrm{~K}$ and $\sigma(\log g) \simeq$ 0.03 dex to reach precisions of $\sim 0.01$ dex in the abundance determinations. Such high precisions may be obtained by analysing EWs of the many Fe lines available in late-type stellar spectra. The $T_{\text {eff }}$ of the model atmosphere is often chosen so that there is no systematic dependence of the derived Fe abundance on excitation potential, $\chi_{\text {exc }}$, of the lines, and $\log g$ may be determined from the requirement that the Fe abundances derived from Fe I and Fe II lines should agree. As the electron pressure in the models is significantly affected by the abundance of Fe and some alpha-capture elements (especially $\mathrm{Mg}$ and $\mathrm{Si}$ ), one must also assure that the models applied have consistent values of $[\mathrm{Fe} / \mathrm{H}]$ and $[\alpha / \mathrm{Fe}]$. This means that the determination of $T_{\text {eff }}$ and $\log g$ is an iterative process, where the final parameters are found by interpolation in a 4-dimensional grid of model atmospheres.

Effective temperatures can also be determined by the infrared flux method (IRFM) or from colour indices like $V-K$ as discussed in detail by Casagrande et al $(2010)$. For groups of nearby stars with similar parameters the precision is $\sim 30 \mathrm{~K}$, but interstellar reddening is a problem for more distant stars. Alternatively, one

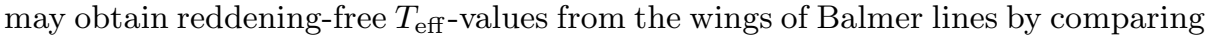
with model atmosphere profiles calculated from the theory of Stark broadening by charged particles and self-broadening by hydrogen atoms (see review by Barklem

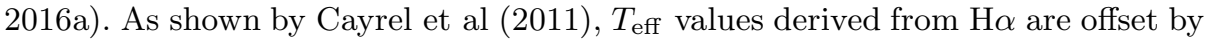
$\sim-100 \mathrm{~K}$ relative to interferometric temperatures based on angular diameter and bolometric flux measurements of solar-type stars (including the Sun). This may be due to problems with the temperature structure of the 1D model atmospheres applied for the analysis of the $\mathrm{H} \alpha$ profiles, as is demonstrated by the fact that the

\footnotetext{
3 In an abundance study of 714 Galactic disk stars (Bensby et al 2014), a record high number of 300000 spectral lines were measured interactively by Thomas Bensby using the IRAF splot task
} 

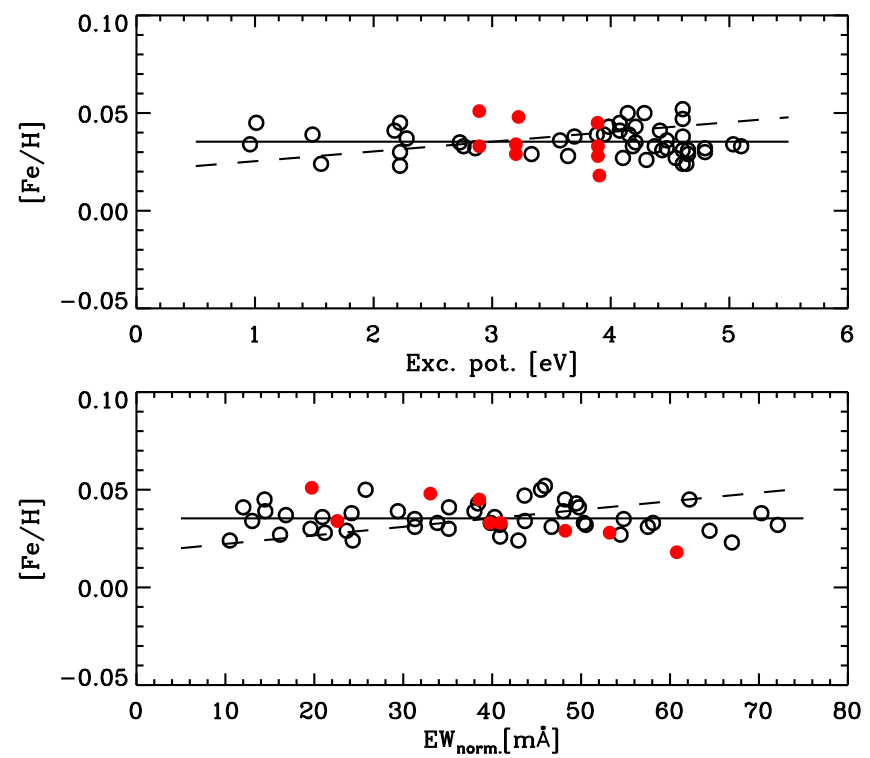

Fig. $2[\mathrm{Fe} / \mathrm{H}]$ derived from EWs in the spectrum of the solar twin HD 183658 as a function of excitation potential (upper panel) and equivalent width normalized to $\lambda=6000 \AA$, i.e. $E W_{\text {norm. }}=E W / \lambda \cdot 6000.0$ (lower panel). Open circles refer to Fe I lines and filled red circles to Fe II lines. The full drawn lines show linear fits to the data for the derived parameters of the star $\left(T_{\text {eff }}=5809 \mathrm{~K}, \log g=4.402,[\mathrm{Fe} / \mathrm{H}]=0.035, \xi_{\text {turb }}=1.04 \mathrm{~km} \mathrm{~s}^{-1}\right)$. The dashed lines show changes in the slopes, if $T_{\text {eff }}$ is decreased by $30 \mathrm{~K}$ (upper panel) and the microturbulence is decreased by $0.1 \mathrm{~km} \mathrm{~s}^{-1}$ (lower panel). Based on data from Nissen (2015).

results obtained depend critically on the assumed mixing-length parameter of the 1D models (Ludwig et al 2009a). Recent 3D non-LTE modelling by Amarsi et al (2018b) leads to better agreement between Balmer-line temperatures and interferometric values of $T_{\text {eff }}$, and it is encouraging that the scatter in the relation between interferometric and $\mathrm{H} \alpha$ temperatures after the offset has been applied is only $30 \mathrm{~K}$ for stars with $5000<T_{\text {eff }}<6400 \mathrm{~K}$ and $-0.7<[\mathrm{Fe} / \mathrm{H}]<0.2$ (Cavrel et al 2011). A similar scatter was found by Ramírez et al (2014) when comparing $\mathrm{H} \alpha$ temperatures of solar twins stars with precise excitation temperatures. Further improvements of the precisions of Balmer line temperatures to say $\sigma\left(T_{\text {eff }}\right) \sim 10 \mathrm{~K}$ may, however, be difficult because the continuum of the observed spectra have to be defined very precisely over regions of $\pm 30 \AA$, which is a considerable fraction of the free spectral range of an echelle-order in a typical spectrograph. For this purpose, low-order grating spectrometers should be applied.

If hydrostatic plane-parallel 1D model atmospheres are used, the effect of gas motions on the equivalent widths has to be described by yet another parameter, the microturbulence $\xi_{\text {turb }}$, normally assumed to be independent of depth in the atmosphere and added in quadrature to the thermal Doppler velocity broadening. The effect of this parameter on abundance determinations is negligible for weak lines, say $E W \lesssim 30 \mathrm{~m} \AA$ in the visual part of the spectrum, but becomes important for lines with $E W \gtrsim 50 \mathrm{~m} \AA$. Hence, $\xi_{\text {turb }}$ may be determined from the requirement 
that the derived Fe abundances should be independent of EW. To avoid degeneracy between the determination of $T_{\text {eff }}$ and $\xi_{\text {turb }}$ from equivalent widths, it is important that $\mathrm{Fe}$ lines in a small interval of $\chi_{\text {exc }}$ have a large range in EW. An example of the technique is shown in Fig.2.

From the $1-\sigma$ errors of the slopes of $[\mathrm{Fe} / \mathrm{H}]$ versus $\chi_{\text {exc }}$ and EW and the errors of the average Fe abundances derived from Fe I and Fe II lines, one can estimate the uncertainty of the atmospheric parameters using error analysis methods, which take into account correlations in the parameter determinations (e.g. McWilliam et al 1995; Epstein et al 2010; Bensby et al 2014). When based on equivalent width measurements in spectra with $R \gtrsim 50000$ and $S / N \gtrsim 400$, precisions in relative values better than $10 \mathrm{~K}$ in $T_{\text {eff }}, 0.02 \mathrm{dex}$ in $\log g$, and $0.01 \mathrm{dex}$ in $[\mathrm{Fe} / \mathrm{H}]$ and $[\alpha / \mathrm{Fe}]$ are estimated (e.g. Meléndez et al 2009; Ramírez et al 2014; Nissen 2015). Comparisons of independent determinations for solar twins confirm these estimates, but it should be emphasized that these small errors are only relevant when stars with similar parameters are compared, i.e. stars having ranges less than $300 \mathrm{~K}$ in $T_{\text {eff }}$ and less than $0.3 \mathrm{dex}$ in $\log g$ and $[\mathrm{Fe} / \mathrm{H}]$. If larger differences are present, systematic errors due to non-LTE and 3D effects may dominate and have to be considered, see Sect. 2.4

An alternative way of determining stellar surface gravities is based on the fundamental relations between gravity, mass, radius, luminosity, and effective temperature of a star, $g \propto \mathcal{M} / R^{2}$ and $R^{2} \propto L / T_{\text {eff }}{ }^{4}$, corresponding to the equation

$$
\log \frac{g}{g \odot}=\log \frac{\mathcal{M}}{\mathcal{M} \odot}+4 \log \frac{T_{\text {eff }}}{T_{\text {eff }, \odot}}+0.4\left(M_{\mathrm{bol}}-M_{\mathrm{bol}, \odot}\right)
$$

where the various parameters are normalized on the solar values. The absolute bolometric magnitude, $M_{\text {bol }}$, can be determined from the apparent magnitude if the distance is known and the bolometric correction can be estimated. For a parallax error of $1 \%$, the induced error of $\log g$ will be about 0.01 dex. With the Hipparcos satellite such small parallax errors have been obtained only for a small fraction of the solar twins, binaries, and stars in clusters, for which high-precision abundances and effective temperatures have been derived. Based on Gaia data, however, the parallaxes of most of the stars studied are expected to become known with accuracies better than $1 \%$. The largest contribution to the error of $\log g$ will then arise from the mass, which is often obtained by interpolating between stellar evolutionary tracks in the $L-T_{\text {eff }}$ diagram. Knowing $T_{\text {eff }}$ and $[\mathrm{Fe} / \mathrm{H}]$ from highprecision spectroscopy, differential masses of solar twins can be obtained to a precision of $\sim 0.01 \mathcal{M}_{\odot}$ (e.g. Ramírez et al 2014) corresponding to an error of 0.005 dex in $\log g$, but in other regions of the $L-T_{\text {eff }}$ diagram the error may be much larger.

Precise surface gravities may also be determined from asteroseismology, i.e. by comparing observed oscillation frequencies with predictions from stellar models. For about 100 main-sequence and subgiant stars having long time series of precise flux measurements obtained with the Kepler satellite, $\log g$ has been determined with a precision better than 0.01 dex (Silva Aguirre et al 2015, 2017) and for Kgiants in the so-called APOKASC programme, $\log g$ is determined with an average precision of 0.014 dex (Pinsonneault et al 2014).

In addition to the methods discussed above two more spectroscopic methods for determining stellar gravities may be mentioned: the use of pressure-sensitive 
wings of strong lines (Blackwell and Willis 1977) and the use of pressure-sensitive molecular lines like $\mathrm{MgH}$ (Bell et al 1985).

The different ways of determining surface gravities make it possible to investigate if variations in other model-atmosphere parameters than $T_{\text {eff }}, \log g,[\mathrm{Fe} / \mathrm{H}]$, and $[\alpha / \mathrm{Fe}]$ play a role when determining precise abundances. One such parameter is the helium abundance, $y=N_{\mathrm{He}} / N_{\mathrm{H}}$, which for example in the MARCS models (Gustafsson et al 2008) is assumed to be $y=0.085$ corresponding to a helium mass fraction of $Y=0.25$ close to the solar surface value determined from helioseismology (e.g. Basu and Antia 2004). Given that atomic diffusion decreases the atmospheric helium abundance as a function of increasing stellar age (the effect is about $10 \%$ for the Sun, e.g. Christensen-Dalsgaard et al 1993) and Galactic chemical evolution increases the initial He abundance as a function of time, it would not be a surprise if young stars have $\Delta Y \sim 0.05-0.10$ relative to the oldest stars. Such differences of the He abundance may lead to a significant effect on spectral lines. For late-type stars, helium does not contribute to opacity and free electrons, but it affects the mean molecular weight and hence the electron pressure in the atmosphere of which, for instance, the strengths of Fe II lines depend. As shown by Strömgren et al (1982), two models with identical temperature structures but different surface gravities, $g_{1}$ and $g_{2}$, and different helium abundances, $y_{1}$ and $y_{2}$ (by number) will have closely similar $P_{e}(\tau)$ relations if

$$
g_{2}=g_{1} \frac{1+4 y_{1}}{1+y_{1}} \frac{1+y_{2}}{1+4 y_{2}}
$$

This means that for two stars with the same $T_{\text {eff }}$, same heavy element composition relative to hydrogen, and same equivalent widths of Fe II and Fe I lines, one will derive a difference in gravity, $\Delta \log g \simeq-0.02$ dex if the difference in He mass fraction is $\Delta Y=0.06$ (corresponding to an increase of the He abundance from $y=0.085$ to $y=0.108$ ). Hence, a comparison of high-precision spectroscopic gravities with gravities based on Gaia parallaxes or seismic data may reveal possible variations in He abundances. In such a comparison, the effects of He abundance variations on derived stellar masses (used for calculating trigonometric gravities) and on seismic gravities should be taken into account. The method may also be applied to determine He abundance differences between stars in a cluster with the advantage that the distances do not enter into the problem. Interestingly, Lind et al (2011) showed that the He abundance difference between a first and a second generation star in the globular cluster NGC 6397 is not very pronounced, i.e. $\Delta Y=0.01 \pm 0.06$.

\subsection{Spectrum synthesis techniques}

Instead of using equivalent widths, one may derive stellar abundances by comparing observed spectra with synthetic model-atmosphere spectra. Pioneering studies were made by Payne-Gaposchkin and Whipple (1940); Baschek (1959); Fischel (1964); Cayrel (1969); Bell (1970). For heavily blended lines, this is the only way to determine precise abundances. An example is provided in Fig. 3 , which shows how the thorium abundance of a very metal-poor $r$-process-rich K-giant star, HD 11544, is determined by Westin et al (2000). Although the Th II line at 4019.14 $\AA$ overlaps with two ${ }^{13} \mathrm{CH}$ lines, the spectrum synthesis yields a fairly precise Th abundance of 


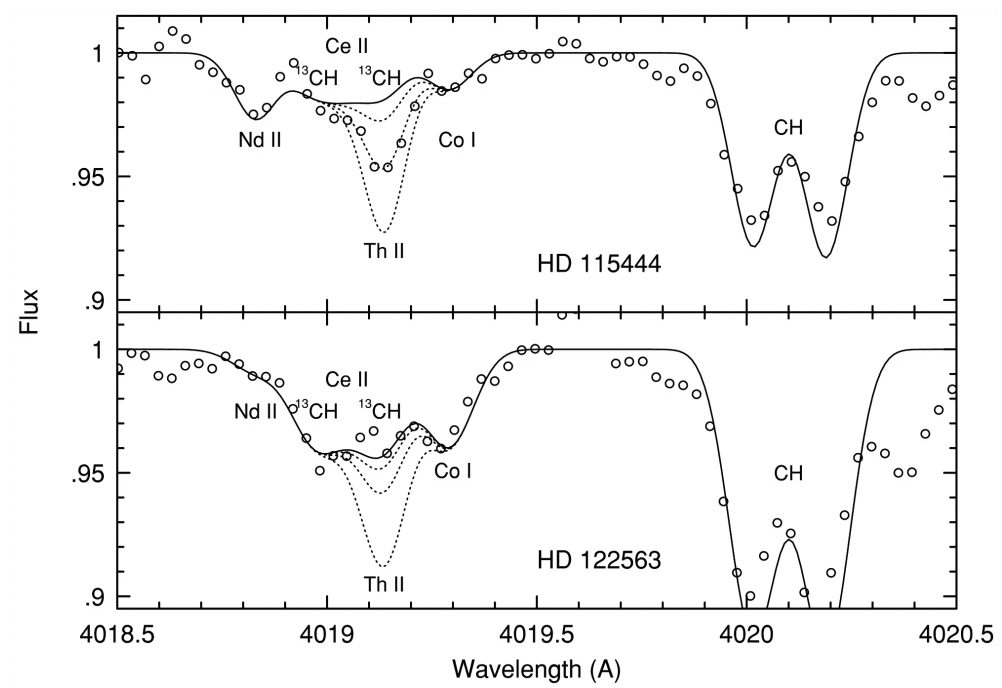

Fig. 3 A high-resolution $(R=60000, S / N \simeq 200)$ spectrum around the Th II line at $4019.1 \AA$ for the metal-poor $r$-process-rich K-giant star, $\mathrm{HD} 115444([\mathrm{Fe} / \mathrm{H}] \simeq-3.0,[\mathrm{Eu} / \mathrm{Fe}]=+0.9)$ in comparison with the spectrum of a metal-poor, $r$-process-poor K-giant star, HD 122563 $([\mathrm{Fe} / \mathrm{H}] \simeq-3.0,[\mathrm{Eu} / \mathrm{Fe}]=-0.4)$. Full drawn lines show synthetic spectra without Th and dotted lines with Th abundances of $\log \epsilon(\mathrm{Th})=-2.48,-2.23,-1.98$ for HD 115444 and $\log \epsilon(\mathrm{Th})=-3.08,-2.58,-2.08$ for HD 122563. Based on spectra from Westin et al (2000).

$\log \epsilon(\mathrm{Th})=-2.23 \pm 0.054$, whereas the Th II line (and the Nd II line at 4018.84 A) is not detected in the comparison star HD 122563. As shown by Westin et al, this may be used to estimate a radioactive age of HD 115444 from the abundance of Th relative to the abundance of stable $r$-process elements.

In the example shown in Fig. 3, the stellar atmospheric parameters were derived from equivalent widths by the method described in Sect. 2.2. The stellar parameters may, however, also be determined by spectrum synthesis techniques. A popular tool for this is the Spectroscopy Made Easy (SME) package (Valenti and Piskunov 1996; Piskunov and Valenti 2017), which can be used to interpolate in a grid of model atmospheres and to calculate synthetic spectra based on a line list, e.g. the Vienna Atomic Line Database (VALD5). Atmospheric parameters and abundances may then be derived by chi-square fitting of observed high-resolution spectra in selected spectral windows. In addition to $T_{\text {eff }}, \log g,[\mathrm{Fe} / \mathrm{H}]$, and microturbulence, the fitting must include parameters for macroturbulent and rotational broadening of spectral lines. These broadening mechanisms do not change the total flux absorbed in a line and have therefore no direct effects on parameters and abundances determined from equivalent widths, although there may be indirect effects related to continuum placement and fitted line profiles or integration limits when measuring the EWs.

An improvement of great significance in synthetic spectroscopy during recent decades has been the precise quantum-mechanical calculation of collision broaden-

\footnotetext{
4 For an element $\mathrm{X}, \log \epsilon(\mathrm{X}) \equiv \log \left(N_{\mathrm{X}} / N_{\mathrm{H}}\right)+12.0$

5 http://vald.astro.uu.se. Note that in many cases it may be proper and important to give a reference to the source of the original data and not only refer to the data base.
} 
ing of atomic lines by Anstee and O'Mara (1991, 1995) and Barklem et al (2000) (see Barklem 2016a, for further references).

Brewer et al (2016) provide a good example of how stellar parameters and elemental abundances can be determined with the SME package. Spectra of $\sim 1600$ F, G and K main-sequence and subgiant stars were obtained with the HIRES spectrograph at KECK Observatory with a resolution of $R \simeq 70000$ and $S / N \gtrsim 100$ for $\sim 75 \%$ of the sample. Synthetic spectra were calculated for specific wavelength regions between 5100 and $7800 \AA$ and fitted to the observed spectra. The strong $\mathrm{Mg}$ I b triplet in the 5150 - $5190 \AA$ region was included, which provides a measure of the surface gravity in addition to the information obtained from the ratio of the strengths of Fe I and Fe II lines, because the absorption in the wings of the $\mathrm{Mg}$ lines increases with gas pressure in the atmosphere. Atomic line data were adopted from VALD with empirical adjustments of $g f$-values, van der Waals coefficients, and wavelengths obtained by fitting the solar flux atlas of Wallace et al (2011) adopting abundances from Grevesse et al (2007) and a microturbulence of $\xi_{\text {turb }}=$ $0.85 \mathrm{~km} \mathrm{~s}^{-1}$. Furthermore, the zero points of $[\mathrm{X} / \mathrm{H}]$ were checked and slightly adjusted using the average abundances derived from 20 spectra of four asteroids observed with HIRES at the same resolution as the stars. As a comment to this procedure, we note that it is not easy to deduce absolute $g f$-values or damping constants by fitting solar spectra with accuracies better than 0.1 dex in view of uncertainties in the solar model, departures from LTE, line blends, etc. In strictly differential analyses for solar-like stars, however, considerably higher accuracies may be achieved, since the effects of many of these sources of error will cancel.

Another example of the use of the SME package is the analysis of GALAH $(R=$ 28000 ) spectra by Buder et al (2018), who determined atmospheric parameters and abundances of 23 elements for a large sample of dwarf and giant stars. For a number of key elements ( $\mathrm{Li}, \mathrm{O}, \mathrm{Na}, \mathrm{Mg}, \mathrm{Al}, \mathrm{Si}$, and $\mathrm{Fe}$ ) non-LTE corrections were included, which makes the derived abundances more reliable.

Spectrum synthesis techniques, when applied to spectra containing partly saturated lines, have the problem that the determination of the microturbulence parameter is partially degenerate with the other parameters $T_{\text {eff }}, \log g$, and $[\mathrm{Fe} / \mathrm{H}]$. When including $\xi_{\text {turb }}$ as as free parameter, Brewer et al (2015) find deviations between seismic gravities and spectroscopic gravities of up to 0.25 dex for a sample of 42 Kepler stars. Assuming instead a constant microturbulence of $\xi_{\text {turb }}=$ $0.85 \mathrm{~km} \mathrm{~s}^{-1}$ as adopted for the solar atmosphere, excellent agreement with the Kepler gravities is obtained, i.e. a rms deviation of only 0.05 dex over a $T_{\text {eff }}$ range from $5000 \mathrm{~K}$ to $6500 \mathrm{~K}$. This suggested constancy of the microturbulence parameter is somewhat surprising, because from analyses of EWs of main-sequence stars, $\xi_{\text {turb }}$ is found to increase by about $1.2 \mathrm{~km} \mathrm{~s}^{-1}$ from $T_{\text {eff }}=5000 \mathrm{~K}$ to $6500 \mathrm{~K}$ and depends also on gravity (e.g. Edvardsson et al 1993; Ramírez et al 2013). Another problem is that the average $[\mathrm{Fe} / \mathrm{H}]$ abundance of stars in the sample of Brewer et al

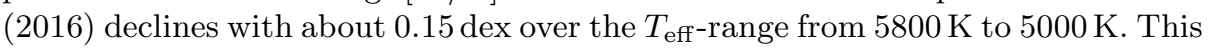
may be related to the adoption of a constant microturbulence and/or deficiencies in the stellar models and the assumption of LTE. Obviously, 3D non-LTE analyses are needed to get more accurate abundances (see Sect. 2.4). However, this does not exclude that very precise differential abundances can be derived for narrow ranges in $T_{\text {eff }}$. From repeated observations of stars having $S / N>100$ spectra, Brewer et al (2016) obtain average std deviations of $12 \mathrm{~K}$ in $T_{\text {eff }}, 0.014$ dex in $\log g$, and $\lesssim 0.01$ dex for the abundance of many elements. 
The APOGEE survey (Majewski et al 2017) is another example of the use of spectrum synthesis techniques in deriving high-precision stellar abundances. During a three-year observing campaign on the Sloan $2.5 \mathrm{~m}$ telescope, near-infrared spectra with $S / N>100$ covering the $H$-band $(1.5-1.7 \mu \mathrm{m})$ with a resolution of $R \simeq 22500$ have been obtained for about 150000 stars. Most of them are intrinsically bright K-giants and due to the relatively low interstellar absorption in the $H$-band, abundances in stars of various populations belonging to the Galactic disk, the bulge, and the halo can be studied over a wide range of distances.

In the APOGEE survey, stellar parameters and abundances of up to 15 elements are derived by comparing observed spectra to a large library of synthetic spectra using a chi-square minimization programme ASPCAP (García Pérez et al 2016). The synthetic spectra are calculated for ATLAS9 model atmospheres (Castelli and Kurucz 2003) and normalized to pseudo-continuum spectral windows in the same way as the observed spectra. The line list (see Shetrone et al 2015) consists of more than 130000 atomic and molecular transitions for which the $g f$-values were adjusted so that high-resolution atlases of the Sun and Arcturus Livingston and Wallace 1991; Hinkle et al 1995) are well fitted. Given that the $H$-band includes many molecular lines from $\mathrm{CO}, \mathrm{CN}$, and $\mathrm{OH}$, parameters for the $\mathrm{C}, \mathrm{N}$, and $\alpha$-element abundances were included in the grid of synthetic spectra in addition to the usual atmospheric parameters. The microturbulence may be obtained from the fitting, but a simple dependence on gravity, as found for a subset of well observed stars, is adopted for K-giants. Instrumental broadening is applied to the calculated spectra taking into account variations in the line spread function over the field of the CCD detectors. Furthermore, a constant Gaussian broadening of $6 \mathrm{~km} \mathrm{~s}^{-1}$ (FWHM) is adopted to take care of macroturbulence. Rotational broadening is assumed to be negligible, which may introduce errors in the derived parameters and abundances for stars having $V \sin i \gtrsim 5 \mathrm{~km} \mathrm{~s}^{-1}$, but this is only the case for a small fraction of red giants.

After a first pass in which the atmospheric parameters of stars are determined from the entire APOGEE spectral band, individual abundances of 15 elements are derived from selected spectral windows containing atomic and molecular lines of the element considered. As discussed by Holtzman et al (2015), there are systematic deviations in $\log g$ relative to seismic gravities and trends in abundances as a function of $T_{\text {eff }}$, but abundances derived for stars in solar metallicity clusters have only a small scatter in narrow ranges of effective temperature. For the best observed elements $\left(\mathrm{O}, \mathrm{Mg}\right.$, and $\mathrm{Fe}$ ), the rms scatter in intervals of $\Delta T_{\text {eff }}=250 \mathrm{~K}$ is about 0.03 dex for cluster stars having APOGEE spectra with $S / N \sim 200$ and for the abundance ratios $[\mathrm{O} / \mathrm{Fe}]$ and $[\mathrm{Mg} / \mathrm{Fe}]$, the scatter is only $\sim 0.02 \mathrm{dex}$ (Ness et al 2018).

High-precision abundances may also be derived from low-resolution $(R \lesssim 6000)$ spectra, if accurate spectral models are available and accurate spectrum normalization can be achieved (Ting et al 2017). In crowded low-resolution spectra most absorption features and pseudo-continuum windows are, however, affected by strong lines sensitive to microturbulence, damping constants, non-LTE, and 3D effects, which makes it difficult to calculate sufficiently accurate synthetic spectra. Other problems are unidentified lines or lines with poorly known wavelengths and $g f$ values. Instead of a direct analysis, low-resolution spectra may therefore be modelled based on a set of parameters and abundances ("labels") available from a 
high-resolution study of a subset of stars. A popular tool for this is The Cannon 6 introduced by Ness et al (2015). It uses the labels of a learning set of stars to find the parameters of a spectral model describing the flux in each spectral pixel as a function of the labels. A good example is provided by Ho et al (2017a, b), who apply The Cannon to transfer labels from APOGEE to $R=1800$ LAMOST (Zhao et al 2012) optical spectra of K-giants using a learning set of $\sim 8000$ stars in common between the two surveys. This has resulted in metallicities $[M / H]$ and abundance ratios, $[\mathrm{C} / \mathrm{M}]$ and $[\mathrm{N} / \mathrm{M}]$ (derived from the $\mathrm{CH}$ and $\mathrm{CN}$ bands in the 4100 - $4400 \AA$ region) for 450000 LAMOST giants with precisions $\lesssim 0.1$ dex relative to the APOGEE values. Furthermore, the ratio between $\alpha$-capture elements and iron, i.e. $[\alpha / \mathrm{Fe}]$, is estimated to have a precision of $\sim 0.03 \mathrm{dex}$ if the LAMOST spectra have $S / N>50$. A drawback of The Cannon is, however, that reliable abundances cannot be determined for stars with atmospheric parameters and compositions that are not well covered by the learning set of stars.

\subsection{Model atmosphere analysis, 3D and non-LTE effects}

In order to interpret observed stellar spectra in physical terms, models of the light emitting regions, the stellar atmospheres, are needed. These are adequately constructed by solving the conservation equations of mass, momentum and energy for a compressible gas flow in the gravity field of the star, with a given energy input at the lower boundary, and radiative losses out to free space from the upper layers. Particular care has then to be exercised in the treatment of the radiative heating/cooling term in the energy equation. At every time step in the integration of the hydrodynamic equations, the equation of radiative transfer has therefore also to be solved, together with the conservation equations, for a considerable number of frequencies in order to represent the total radiative flux properly.

Although a non-trivial task numerically, in particular due to the non-linear character of the momentum (Navier-Stokes) equations, it is today quite possible to construct such models, which may then for solar-type dwarfs be just a section of a stellar atmosphere (a "box in a star") with adequate boundary conditions, where the time-development of the state of the gas in the box is simulated. When calculating the integrated stellar spectrum, the spectra of a number of such states may be co-added to represent the total flux from the star. During the last 30 years, the methods to construct such 3D models have been successfully advanced by Nordlund and collaborators (Nordlund and Stein 1990; Stein and Nordlund 1998; Asplund et al 2000b; Collet et al 2011; Trampedach et al 2013; Magic et al 2013) and the resulting STAGGER code is now used for producing extensive grids of models. Important and independent work along similar lines is also made by a group behind the $\mathrm{CO}^{5} \mathrm{BOLD}$ code Wedemever et al 2004; Ludwig et al 2009b; Frevtag et al 2012). In this latter group, also similar models of the full star (a "star in a box") have been constructed for supergiants and AGB stars, where the photospheric granules are of sizes not very different from that of the full star so that "box in a star" models would be inadequate.

6 The name refers to Annie J. Cannon, who from 1911 to 1915 classified 225300 stars by visual inspection of Harvard objective prism photographic plates and later published the results as the Henry Draper (HD) catalogue 
At comparison with observations, these 3D hydrodynamic models have been quite successful. The observed asymmetries of spectral lines, due to the convective motions of the gas, as a function of line depth and excitation were reproduced (Nordlund and Dravins 1990; Dravins and Nordlund 1990) and so was the variation of line strength with transition probability ("the curve of growth", Asplund et al $(2000 \mathrm{~b})$ ). The latter achievement was a major one from the perspective of abundance analysis, since it freed the analysis from the suspect "microturbulence velocity", a parameter used to fit the strength of partly saturated spectral lines in traditional studies (as mentioned already in Sect. 2.2) with no very clear physical definition. In fact, the 3D models showed that the observed line broadening ascribed to "microturbulence" was not mainly due to small-scale turbulence but sooner the result of velocity gradients in large-scale granular motions. One further impressive achievement of the 3D models was the demonstration that they reproduced the various abundance measures for oxygen in the Sun, whether high-excitation or low-excitation atomic lines or molecular ones, with one consistent abundance, (see Pereira et al 2009, and references therein). Other important achievements are the excellent agreement between predictions from 3D models and observations of centre-to-limb variations of continuum intensity (Pereira et al 2013) and iron lines (Lind et al 2017).

Also, in another very important respect, the detailed 3D hydrodynamic models were able to free the analysis from more ad-hoc parameters that are as questionable as the microturbulence velocity. The traditional way of calculating the convective flux was namely based on the "Local Mixing Length Theory", with roots in early work by Prandtl (1926, cited by Biermann (1932)), Biermann (1932), and Vitense (1953), which, when applied to stellar envelopes and atmospheres contains several free parameters, notably the "mixing length" (Henvev et al 1965; Gustafsson et al 1975) with definitions that are only partially possible to relate to the real physical situation. The hydrodynamic 3D models are in practice not dependent on such parameters. One might ask whether the maximum box size, or the minimum grid spacing, of the numerical representation of the atmosphere would not after all be new significant free parameters. Detailed comparisons with different spatial resolutions have shown, however, that this is not the case (Asplund et al 2000a).

When calculating the spectrum from the model atmospheres for comparison with observed spectra, the interaction of the radiation with the gas must be properly represented. In the general case this is a complex problem since the number densities in the lower and upper states of a spectral line are affected by the radiation, and moreover not only by the radiative field in the observed spectral line itself but by the populations in every state of that element (atom, ion, or molecule) that directly or indirectly, through radiative or collisional transitions, significantly affect these states of the line. The fact that the real populations of the atomic states are not only the result of the statistical equilibrium of the particular atom but also of the equilibrium of other atoms, ions and molecules, for instance of the relevant electron donors, means that in an abundance analysis "corrections for non-LTE" must in principle be extended beyond the study of individual atoms in a given model atmosphere. In order to handle this problem, the full statistical-equilibrium equations have to be solved simultaneously for every atomic state of significance and every spatial point in the model. In these equations, the rate of transitions to any particular state is set equal to the rate of transitions from it. In this way, the number densities of different atomic states are linked together in a large system 
of linear equations, and moreover, also the radiative fields in the corresponding transitions have to be considered when calculating the radiative transition rates. Since the radiative fields are non-local, the radiative rates couple also the different spatial points in the model together. The number of important states for an atom is excessive and the number of important transitions (including both radiative and collisional ones) may be several thousands or more. Thus, this is clearly a complex problem. It gets even more difficult since vast amounts of atomic data, including radiative transition rates and collision transition rates due to both atomic and electronic collisions, are needed and often missing.

For several decades, a major problem in statistical-equilibrium calculations for the modelling of stellar atmospheres and spectra has been the the lack of realistic data for the cross sections of collision processes, both as regards excitation of atoms and molecules by collisions with electrons and with hydrogen atoms. Until recently, only semi-empirical methods have been available which on good grounds were expected to lead to collision cross sections that could be in error by even orders of magnitude. During the last decade, however, this situation is greatly changed. Barklem, Belyaev and collaborators have contributed realistic cross sections for hydrogen collisions with atoms of $\mathrm{Li}, \mathrm{O}, \mathrm{Na}, \mathrm{Mg}, \mathrm{Al}, \mathrm{Si}, \mathrm{Ca}, \mathrm{K}$, $\mathrm{Fe}$, and $\mathrm{Rb}$, and cross sections for electron collision excitation of $\mathrm{Li}, \mathrm{O}$ and $\mathrm{Mg}$, see the comprehensive review by Barklem (2016a) and references therein. Most of these new data have now been applied in spectrum analyses and greatly improved the validity of the results. Of particular importance are the calculation of cross sections for hydrogen collisions with iron atoms by Barklem (2018), already applied for a more accurate determination of the solar iron abundance by Lind et al (2017).

At present, relatively complete and accurate model atoms for calculations of spectra from model atmospheres have been assembled for about 20 different atomic species. However, for several of these model atoms, important data like hydrogen collision cross sections and photoionization cross sections are only rough semi-empirical estimates that urgently need improvement. Considerable effects on abundance determinations have been found when applying statistical equilibrium instead of older work in which Local Thermodynamic Equilibrium (LTE) was assumed (that is that all energy and ionisation states were populated according to Boltzmann-Saha statistics with one local depth-dependent temperature $T$ ). Thus the "non-LTE effects" on abundances are often significantly greater than the precision requirements discussed here of about 0.03 dex or better. In particular for metal-poor star where the over-ionization by the hot ultraviolet radiation may severely reduce the content of neutral atoms, the estimates by different groups of non-LTE effects may still depart significantly, e.g. reflecting differences in model atoms, atomic data, model atmospheres with different UV fluxes, etc. One example are the recent results by Shi et al (2018) and Korotin et al (2018) for copper in metal-por stars which differ considerably probably as a result of the use of different cross sections. Similar uncertainties may plague the analyses of several elements in the iron group such as Mn (see e.g. Sneden et al 2016). Critical comparisons of methods and results of different groups should routinely be carried out.

Most of these statistical equilibrium calculations have, however, as yet been made only for plane-parallel models as discussed below. For 3D models, methods of different sophistication have been applied in the radiative-transfer calculations. In the " $\langle 3 \mathrm{D}\rangle$ approximation", the physical properties at each horizontal layer in the $3 \mathrm{D}$ model is first averaged before the spectrum is calculated. In the " $1.5 \mathrm{D}$ 
approximation", the radiative field is only integrated in the vertical direction, while in "full 3D" departures from plane-parallel stratification are considered in all directions. This step, to the "full 3D" (3D, below) has been found to be significant in several cases (see e.g. Amarsi et al 2016b; O'Malley et al 2017), in particular for metal-poor models where the transparent gas may let radiation in "side-ways" from hotter regions, e.g. able to photo-excite/ionize the atoms of interest. Obviously, the $\langle 3 \mathrm{D}\rangle$ models are not to be recommended for accurate analyses of very metalpoor stars, at least not for stars close to the turn-off point where the non-local hot ultraviolet radiation may importantly affect the atoms in the cooler gas sideways as well as vertically. Instead, in non-differential analyses, full 3D models with spectrum calculations in statistical equilibrium should be used.

The model spectra described above have been calculated a posteriori, on the basis of 3D models where the radiative field needed for the energy balance equation of the model (as well as the thermodynamics of the gas) were calculated assuming LTE. There is still some time before one can expect fully consistent model atmospheres to be available where this LTE assumption is lifted also at the calculation of model structure, and not only of the final spectrum. The most important steps towards consistency, seem, however, to have been taken: the modifications to expect from fully non-local radiation fields in $3 \mathrm{D}$ models are probably smaller.

Since 40 years, the model stellar atmospheres used in most abundance analyses have, however, been far more primitive and much less realistic than those described above: they have been based on a number of simplifying and partly unrealistic assumptions: plane parallel (or for larger stars spherically symmetric) stratification of the gas in hydrostatic equilibrium, LTE, and energy balance. Here, the radiative energy flux was calculated by the equation of radiative transfer with the Planck function $B_{\nu}(T)$ as the source function - if need be supplied with a scattering term. Added to that is the convective flux according to the Local Mixing-Length "Theory". These 1D models have been calculated in extensive grids extending over large ranges in fundamental parameters including chemical composition. The major effort behind them has been directed towards the consideration of the collective effects of millions of spectral lines ("blanketing") on the energy balance and the spectrum, and indeed, these effects are very considerable on the temperature structures and the spectra, in particular for cool stars. The most used grids of this type, that are still regularly applied in abundance analyses, are the ATLAS9 grid (Kurucz 1979; Castelli and Kurucz 2003), the MARCS grid (Gustafsson et al 1975, 2008) and the NextGen-grid (Hauschildt et al 1999a, b). The latter models were calculated with the PHOENIX programme which has also enabled the construction of models where the LTE assumption is not made.

The usefulness of the more primitive, and much more easily handled, 1D models in abundance analysis has been explored in a number of comparisons with results from 3D models. The early application to abundance analysis of 3D and nonLTE modeling for solar-type stars was reviewed by Asplund (2005). Until very recently, the application of 3D non-LTE models have mainly been made for solartype stars, including more metal poor ones with temperatures and gravities not too different from the solar values. A general result is that the differences from standard analyses are often greater than $0.04 \mathrm{dex}$, and in particular for metalpoor stars considerably greater than so. Thus, if the ambition is to determine abundances with high accuracies, one should not use the 1D models without a very careful consideration of the systematic errors involved. 


\subsection{Differential analyses}

One way of minimizing the effects of model errors is to perform a differential analysis relative to the Sun, or some other standard star, hoping that the differences between the programme stars and the standard star can be modelled accurately enough by 1D models. (In fact, a differential analysis is anyhow recommendable in view of the fact, mentioned in the Introduction, that in an absolute analysis, even for the Sun itself, it is as yet hard to achieve abundance errors smaller than 0.04 dex.)

Schematically, we may then write any estimate $E$ of the logarithmic abundance ratio $[\mathrm{A} / \mathrm{B}]$ for a programme star $\mathrm{P}$, determined differentially relative to a standard star $\mathrm{S}$ as

$$
E[A / B]^{P}=[A / B]_{a}^{P}-[A / B]_{a}^{S}+[A / B]_{r}^{S},
$$

where an index $a$ denotes an approximate model (e.g., LTE and 1D) and $r$ a more realistic one, such as a $3 \mathrm{D}$ model with statistical equilibrium calculations of the spectrum. A key question is then how different the parameters of the programme star may be from that of the standard star if the error in the estimated abundance ratio should be kept smaller than a certain value, say 0.04 dex. Another, related question is how the fundamental parameters of $\mathrm{P}$ and $\mathrm{S}$ are to be determined, in particular in the fitting of the approximate models, for which the parameters in themselves as estimated from the model spectra may not be correct per se. Although these questions can certainly not be answered fully today for stars located anywhere in the parameter space, there are enough of analyses for solar-like stars, including metal-poor ones, to allow some conclusions.

In an important series of papers Bergemann et al (2012); Lind et al (2012); Amarsi et al (2016b); Lind et al (2017), developed model atoms for Fe I and Fe II with hundreds of energy level and thousands of radiatively-allowed transitions, and applied these in 3D STAGGER models of six standard stars with different fundamental parameters, to study the effects on iron lines and resulting iron abundances when realistic model atmospheres are used. We may consult these results to explore the errors of 1D LTE differential analyses (note, here the word is not used in a strict sense since different spectral lines will have to be used for stars with great abundance differences). For the Sun and Procyon (the latter with estimated parameters $T_{\text {eff }}=6543 \mathrm{~K}, \log g=3.98$ and $\left.[\mathrm{Fe} / \mathrm{H}]=-0.03\right)$ it is found that the iron abundances are raised from standard 1D LTE values by typically 0.03 dex for the Sun (dependent on which lines were used in the standard analysis) when a $\langle 3 \mathrm{D}\rangle$ non-LTE analysis is carried out. For Procyon, the effect is an increase of about 0.06 dex. Hence, for the span of $700 \mathrm{~K}$ between Procyon and the Sun, a differential 1D LTE analysis would still have worked but marginally so, if the errors required in $[\mathrm{Fe} / \mathrm{H}]$ are to be $<0.04$ dex. A full 3D non-LTE analysis may in fact lead to smaller effects as suggested from the solar analysis by Lind et al (2017). It is worth noting that the differential effects for these stars, as long as reliable $\log g$ values are known, are much smaller for Fe II lines than Fe I lines. Also, the errors in LTE analyses of $[\mathrm{Fe} / \mathrm{H}]$ are partially compensated for if consistent LTE excitation equilibria are used for determining the effective temperature.

In addition to the Sun and Procyon, four metal-poor standard stars were discussed by the same authors. The model parameters of these stars are given in Table 1. As is seen, the errors in $[\mathrm{Fe} / \mathrm{H}]$ in a differential analysis for the metal-poor stars 
Table 1 The logarithmic iron abundances relative to the Sun, $[\mathrm{Fe} / \mathrm{H}]$, for four metal-poor stars derived in 1D, LTE analysis, and obtained by Amarsi et al (2016b) using STAGGER models and statistical-equilibrium calculations of the spectra in 3D, respectively. Given are also estimated statistical and systematic errors in the latter $[\mathrm{Fe} / \mathrm{H}]$ determinations.

\begin{tabular}{lrrrr}
\hline & HD84937 & HD122563 & HD140283 & G64-12 \\
\hline$T_{\text {eff }}$ & 6356 & 4587 & 5591 & 6435 \\
$\log g$ & 4.06 & 1.61 & 3.65 & 4.26 \\
{$[\mathrm{Fe} / \mathrm{H}](1 \mathrm{D}, \mathrm{LTE})$} & -2.10 & -2.60 & -2.42 & -3.24 \\
{$[\mathrm{Fe} / \mathrm{H}](3 \mathrm{D}, \mathrm{non}-\mathrm{LTE})$} & -1.96 & -2.49 & -2.31 & -2.94 \\
$\sigma$ (stat) & 0.02 & 0.11 & 0.03 & 0.06 \\
$\sigma$ (syst) & 0.04 & 0.14 & 0.03 & 0.06 \\
\hline
\end{tabular}

will be considerably greater than those discussed in this paper. A closer look at the results of Amarsi et al (2016b) demonstrates the intricacy of the interplay between the different effects. E.g., the iron abundance is raised when non-LTE replaces LTE (mainly due to "overionization" of the neutral atom). If the 1D model is replaced by a $\langle 3 \mathrm{D}\rangle$ model, there is again a small increase in the abundances derived, both if the spectrum is calculated in non-LTE and if LTE is assumed. However, if a full $3 \mathrm{D}$ model is used in the spectrum calculations, the non-LTE abundances increase further while the abundances derived under the LTE assumption decrease significantly.

Iron is important, not only for its rich spectrum and relatively high abundance in solar-type stars. It is also one of the dominating electron donors, and thus affects the continuous opacity through $\mathrm{H}^{-}$. For the additional important electron donor in solar-type stars, Mg, Bergemann et al (2017) found $\langle 3 \mathrm{D}\rangle$ non-LTE corrections of 1D LTE values of about 0.01-0.03 for the Sun (depending on solar model and lines used) and corrections for Procyon of about 0.10. Thus, errors on the order of 0.01 per $100 \mathrm{~K}$ will result in standard analyses of solar-type stars relative to the Sun; however, just as for iron abundances it seems that most of those errors will be compensated for if iron-atom excitation equilibria from standard 1D LTE models are used for the temperature estimate. For the metal-poor stars, however, Bergemann et al (2017) find corrections due to 3D that contribute negatively to $[\mathrm{Mg} / \mathrm{H}]$ while the non-LTE values increase the values - altogether, these lead to positive corrections for stars like HD 84937 and HD 140283, while the $3 \mathrm{D}$ effects dominate with negative corrections for HD 122563. The errors may be up to 0.10 dex in measures relative to the Sun.

In addition to these principal difficulties to bridge great abundance gaps in differential 1D LTE analyses, there is another more practical one: the spectral lines can be of such different strengths for the standard stars, say the Sun, and the programme stars. Therefore, one generally has to take several steps via intermediate standards, using different sets of spectral lines for determining the abundances of these relative to the primary standard and to the programme star. That introduces extra errors e.g. via the determination of the microturbulence parameter, which may require a more detailed 3D non-LTE modelling also of the intermediate standard. In practice, it is therefore very difficult to reach accuracies better than 0.04 dex relative to the Sun for extreme metal-poor stars. However, it is quite feasible to determine precise abundances of these latter stars relative to another metal-poor star then used as a standard. 
For silicon, also an important electron donor in particular for stars slightly hotter than the Sun, statistical-equilibrium calculations by Bergemann et al (2013) and Mashonkina et al (2016) suggest that the differential non-LTE effects are very small for solar-type stars (see also Nissen et al 2017). We also note that Amarsi and Asplund (2017) find small 3D effects in the solar case, using statisticalequilibrium calculations and 3D models.

Motivated by the significance of oxygen as the third most abundant chemical element in the universe, and its origin in massive core-collapse supernovae ( $\mathrm{SNe}$ ) with a short evolution time scale which makes it an important element in the study of galaxy chemical evolution, considerable efforts have been made in exploring the errors made in oxygen abundance analyses with standard 1D models. From the comprehensive study with a 22 level atom (43 radiative transitions), in 3D models, by Amarsi et al (2016a), one finds that the relatively easily accessible $7773 \AA$ triplet should not be used differentially in 1D LTE analyses across larger effective-temperature intervals than $50 \mathrm{~K}$ or $0.1 \mathrm{dex}$ in $\log g$, if the systematic abundance errors are to be smaller than 0.04 dex. The alternative criteria, the high-excitation O I $6158 \AA$ line and the [O I] $6300 \AA$ and $6363 \AA$ forbidden lines are less problematic (although quite weak and therefore difficult to measure at low and high temperatures, respectively) but these lines are more sensitive to 3D effects and difficulties in placing the continuum, and, for the forbidden lines, plagued by blends, including the wide and shallow Ca I auto-ionization line across the $6363 \mathrm{~A}$ line. If these blends can be controlled, the forbidden lines should lead to small errors, even across gaps as wide as $500 \mathrm{~K}$ in $T_{\text {eff }}$ or $0.5 \mathrm{dex}$ in $\log g$ for solar-type stars.

However, the situation may be more problematic for other chemical elements. One example is aluminium which has recently been studied with $\langle 3 \mathrm{D}\rangle$ models in non-LTE for a 42 level atom by Nordlander and Lind (2017). While the differences between $\langle 3 \mathrm{D}\rangle$ non-LTE results and 1D LTE results are small for the Sun as well as for the dwarf HD 22879 with solar temperature but $[\mathrm{Fe} / \mathrm{H}]=-0.86$, and in fact also for the Pop I K0 giant Pollux, they increase to 0.4 dex for the metal poor stars HD 140283 and HD 84937. Almost all this effect is due to overionization by the hot radiation released from deeper layers in the transparent gas in the metalpoor stars. This depletes the lower state of the only useful line for metal-poor stars, the resonance line at $3961 \AA$. For the more metal-rich stars several lines are possible to use, and this illustrates a problem which certainly makes strictly differential analyses difficult when metal-poor stars are compared with much more metal-rich ones: the weights given to different abundance criteria have in many cases to be shifted. The $3 \mathrm{D}$ effects on the $\mathrm{Al}$ lines were considered after spatial and temporal averaging, except in the solar case where a more detailed treatment was carried out. This, however, may not be very problematic since the $3 \mathrm{D}$ effects seem less important in this case than the departures from LTE.

For sulphur, the errors in differential analyses should be small, according to the 1D statistical-equilibrium calculations by Takeda et al (2005). For calcium, however, Mashonkina et al (2017) has used $63+37$ level model atoms for Ca I and Ca II respectively, allowing for charge-transfer and hydrogen-atom collisions rates calculated with cross sections from quantum-mechanical calculations by Barklem (2016b). The error for Procyon when analysed in 1D LTE relative to the Sun is small for Ca I, while the abundance is found to be underestimated by 0.08 dex when high-excitation Ca II lines are used. If metal-poor stars like HD 84937 or HD 140283 
are compared in a 1D LTE analysis with Procyon or the Sun, the systematic errors due to the LTE assumption will be typically -0.2 dex. For titanium, the model atom used by Bergemann (2011) has 216+77 levels for TiI and Ti II, respectively, and more than 4000 transitions. The abundance effects in $[\mathrm{Ti} / \mathrm{H}]$ for metal-poor stars like HD 84937 and HD 140283 are typically underestimates by 0.15 dex when LTE is assumed instead of non-LTE for Ti I, while they are close to zero for Ti II. The corresponding absolute errors for the Sun are typically $1 / 3$ to $2 / 3$ as great, depending on the collision cross sections adopted.

In summary, we conclude that the systematic errors made in 1D LTE differential analysis admit spans between the programme stars and the standard star of about $500 \mathrm{~K}$ for some elements, provided that care is taken in selecting the abundance criteria. However, this is not possible for all elements. For Pop II stars the systematic errors in 1D LTE analyses relative to Pop I stars with similar temperature and surface gravities are as a rule greater than $0.04 \mathrm{dex}$, and the analyses must be corrected using 3D non-LTE models. Alternatively, one may perform a standard analysis relative to another Population II star, and spend particular efforts in analysing that star with detailed models. It should finally be noted that even a strictly differential LTE analysis between two stars with similar fundamental parameters may lead to significant systematic errors due to different non-LTE effects for elements with considerably different abundances in the two stars, especially if saturated lines are used.

\subsection{Effects of magnetic fields}

In the discussion of 3D and non-LTE effects on abundance determinations in the preceding sections we have neglected the possible effect of magnetic fields. The quiet Sun is, however, known to be magnetic, and so are probably all stars. The magnetic fields are expected to affect the stellar spectra, both through the magnetic broadening of the spectral lines ("direct effects") and through forces on the stellar plasma leading to different dynamic and thermodynamic properties of the spectrum-forming gas (indirect effects). Fabbian et al (2012) explored these effects on the solar Fe spectrum, using MHD simulations by the 3D Stagger code, starting from a uniform vertical magnetic field configuration, and with LTE synthetic spectra with Zeeman splitting considered. The spectra were compared with results using non-magnetic models (i.e. pure HD simulations) with the same effective temperatures and surface gravities, and no Zeeman splitting. The authors found that the indirect effects are generally the more important ones for neutral Fe lines, and may lead to an underestimate of the solar Fe abundance by typically about $0.05-0.1$ dex. This is due to the weakening of the lines in the line-forming regions caused by the hotter gas in the magnetized atmosphere. The effect is counteracted but not fully compensated for by the direct effects of Zeeman broadening of the spectral lines. In a subsequent paper Fabbian and Moreno-Insertis (2015) studied the case of the $\mathrm{O}$ I lines and again found that an abundance determination using non-magnetic models would lead to underestimates by a few to several centi-dex.

Shchukina and Trujillo Bueno (2015) challenged the results of Fabbian et al. by a study of Fe spectra from 3D models with and without magnetic fields assuming that the former are dominated by small-scale dynamo action (with the net magnetic flux being zero). Also for solar $\mathrm{C}, \mathrm{N}$, and $\mathrm{O}$ spectra the effects were found 
to be negligible (Shchukina et al 2016). This conflict was resolved by Moore et al (2015), who demonstrated that the assumed field geometry is a key factor: a localdynamo magnetic field gives one order of magnitude smaller effects on abundances than a larger-scale vertical field. Although the issue concerning the true effects on photospheric spectra is not quite resolved, the impressive success for 3D nonmagnetic HD simulations in reproducing solar observations has led Pereira et al (2013) to suggest that the non-magnetic solar models should be preferred. It is not clear to which extent this conclusion is valid for other types of stars.

We also note that the small corrections on derived solar Fe abundances from including small scale magnetic fields with no net magnetic flux are not totally negligible in discussions of very precise abundances of solar twins. Shchukina and Trujillo Bueno (2015) found an average correction of +0.016 dex for Fe I lines, but for low-excitation lines formed in the upper photosphere the correction reaches $\sim+0.05$ dex. Considering that the magnetic field configuration and strength may vary between solar twins, there could be significant magnetic effects on derived differential abundances. Furthermore, the value of $T_{\text {eff }}$ derived from the excitation balance of Fe I lines may be affected, because the magnetic correction depends on excitation potential. The correlation between $\mathrm{Ba}$ abundances derived from a $1 \mathrm{D}$ model atmosphere analysis of the strong Ba II $5853 \AA$ line and the chromospheric activity for young solar twins (Reddy and Lambert 2017) may be connected to such effects, although the authors attribute the correlation to the neglect of enhanced micoturbulence in the upper atmospheric layers of active stars (see Sect. 6.1).

Observational evidence for a connection between stellar activity and equivalent widths of spectral lines have been known since long. Recently, Flores et al (2016) measured the Ca II H\&K and $\mathrm{H} \alpha, \mathrm{H} \beta$ Balmer lines in about 300 high-S/N HARPS spectra of the solar twin HD 45184 observed from 2003 to 2014 and showed that this star with an age of $\simeq 2.7$ Gyr has an activity cycle of 5.14 yr. Strong Fe II lines formed in the upper photosphere were found to be modulated in their EWs over the activity cycle with an amplitude of $\sim 1.5 \mathrm{~m} \AA$ and there is also evidence for variations of Ti II and Ba II lines. This suggests that at some level there are effects of chromospheric activity and associated magnetic fields on derived abundances, but further studies are required to see if this is a significant problem for obtaining abundances of solar twins with a precision of $\sim 0.01$ dex.

\subsection{Cool stars}

The spectra of early and intermediate $\mathrm{K}$ stars are still dominated by atomic lines, and the continuum may be located in between the lines at least in the red and near-infrared spectral region. Also, the modeling of the atmospheres of dwarfs, subgiants and giants of these types is nowadays fairly advanced (see in particular the 3D Stagger grid published by Chiavassa et al (2018) and references therein). For these stars, with spectra of high quality, analyses of quite high precision relative to a proper standard star of similar spectral and luminosity type are possible and are encouraged.

The crowded visual spectra of late K, M and C stars, where the spectral continuum may be hard or impossible to locate, make however high-precision abundance analyses more difficult. This problem can be handled for late $\mathrm{K}$ and early $\mathrm{M}$ stars by using red and infrared spectra where the continuum in places is still possi- 
ble to trace. New detectors, spectrometers, models and not the least molecular and atomic data have led to great progress in this area (for an early review, see Gustafsson 1989) but it is still difficult to reach the high accuracy discussed in the present review. For still cooler stars, the millions of weak lines from polyatomic molecules, like $\mathrm{H}_{2} \mathrm{O}$ for late $\mathrm{M}$ stars and $\mathrm{HCN}$ and $\mathrm{C}_{2} \mathrm{H}_{2}$ for $\mathrm{C}$ stars, change the continuum to an apparent quasi continuum. This requires analysis by synthetic spectra which is dependent on the availability of sufficiently accurate molecular data (wavelengths, transition probabilities, dissociation energies and for statisticalequilibrium calculations, collision cross sections, photo-dissociation cross sections, etc.). Simultaneous determination of the abundances relevant for the molecular equilibrium should be carried out. Another problem for $\mathrm{M}$ and $\mathrm{C}$ stars, which must be considered is the blanketing effects of the molecules on the atmospheres; thus, for these stars the abundance analysis and the model atmosphere construction should be done by iteration, or rely on interpolation in vast grids of models with different sets of abundance data.

Another matter of great significance in the analysis of red giant stars is the accurate determination of stellar fundamental parameters like effective temperature, and, in particular, surface gravity. In a standard spectroscopic analysis of an early $\mathrm{K}$ giant, errors in abundances derived for neutral elements smaller than 0.04 dex require errors in $\log g$ smaller than 0.08 to 0.20 dex, depending on the element in question. This accuracy has been hard to achieve with pure spectroscopic methods. Now, however, with the possibility to use astero-seismological methods built on high-precision photometry (e.g. Pinsonneault et al 2014; Takeda and Tajitsu 2015), including observations of convectively driven brightness variations which also scale with stellar surface gravity (Kallinger et al 2016), surface gravities of much higher accuracy are possible to obtain for these stars. Given this possibility, one may now argue that at least for the numerous early-type $\mathrm{K}$ giants, many of which are He core-burning "clump stars", it should be possible to reach a quite high abundance accuracy in differential analyses relative to a standard star of similar spectral type. For later K giants, and certainly for M giants, line-blending as well as continuum "veiling" are aggravating the situation. Also, the model errors may be expected to be more severe, the later and thus higher up on the giant branch the stars are located, where low surface gravities and low pressures enhance departures from LTE and spherical symmetry. These problems are also illustrated by considerable and individual microturbulence parameters which may lead to systematic errors for saturated spectral lines as long as realistic 3D model atmospheres are not used.

For cooler giants and AGB stars, the gravity estimates in the analyses have often been based on assumed absolute magnitudes and masses. In order to reduce errors in $\mathrm{C}$ or $\mathrm{O}$ abundances for carbon stars to less than about 0.04 dex, one needs to know the parallaxes with an accuracy of about $10 \%$. It remains to be seen how much Gaia parallaxes will improve this situation. Anyhow, uncertainties in model atmospheres is still a major worry for these stars, and hardly admit high accuracies, even if strictly differential approaches are attempted. Such analyses are problematic for these stars, in view of their pronounced individuality.

Pioneering studies of $\mathrm{C}, \mathrm{N}$, and $\mathrm{O}$ compositions of $\mathrm{M}$ stars were made by Spinrad and Vardva (1966), Lambert et al (1984), and Tsuii (1985). A first detailed study of CNO, Fe-peak and s-element abundances of M and MS stars was presented by Smith and Lambert (1985). It was based on near-infrared and in- 
frared high-resolution spectra, selected from wavelength regions only marginally affected by blending TiO bands. The signatures of dredge up of CN-processed material were shown, and enhancements of $s$-process elements shown for the MS stars. The analysis was differential relative to the $\mathrm{K}$ giant $\alpha \mathrm{Tau}$, and the errors in the abundances relative to that star were typically estimated to 0.15 dex.

Vieira (1986) pioneered the use the J band (1.1-1.4 $\mu \mathrm{m})$ in analysis of Mstar spectra and Önehag et al (2012) and Lindgren and Heiter (2017), see also Lindgren et al (2016), performed high-resolution analyses of M dwarf spectra in this band, where the severe blending effects of $\mathrm{TiO}$ are limited. They have shown that a high internal accuracy can be achieved, and that the resulting metallicities agree relatively well for some M-type dwarfs in visual binaries with those of the hotter, and thus more safely analysed primaries. At least for earlier dwarfs than $\mathrm{M} 4$, errors in $[\mathrm{Fe} / \mathrm{H}]$ smaller than 0.1 dex are obviously possible. Similar work has been undertaken by Vevette et al (2017), who estimated fundamental parameters, including $T_{\text {eff }},[\mathrm{Fe} / \mathrm{H}]$, and $[\mathrm{Ti} / \mathrm{Fe}]([\alpha / \mathrm{Fe}])$ for $29 \mathrm{M}$ dwarfs from KeckII/NIRSPEC spectra at a resolution of 25000 . These were calibrated semi-empirically using PHOENIX model atmospheres (Allard et al 2012) and synthetic spectra, and, as regards the abundances, a set of wide binaries with $M$ dwarfs and primaries of FGK-type. For the latter high-resolution spectra were analysed spectroscopically with standard methods. The basic assumption made was that the components in the binaries have identical chemical composition. The formal accuracies are estimated to be about $60 \mathrm{~K}$ in $T_{\text {eff }}$ and 0.05 dex in the abundance ratios, but more independent comparisons are needed to verify that the estimates are free from more systematic errors.

Examples of such studies are those of Rvde et al (2016) of 28 M-giants in the Galactic bulge, and Nandakumar et al (2018) of 72 Inner Galactic Bulge M giants where the high extinction also motivates studies in the $\mathrm{K}$ band at $2.1 \mu \mathrm{m}$. Here, $\mathrm{Fe}, \mathrm{Mg}$, and $\mathrm{Si}$ abundances were measured, although with typical errors estimated to be about 0.15 dex. Yet another example is the study of abundances of $\mathrm{M}$ giants in the Galactic Center Nuclear Cluster by Do et al (2018) who found abnormal Sc, V, and Y abundances, again from K-band spectra. Their interpretation of the strong Sc I lines in terms of high abundances have, however, been questioned by Thorsbro et al (2018) since even nearby M giants are found to show similarly enhanced K-band Sc I lines, suggesting that they are affected by departures from LTE. Even for the warmer N-type carbon stars, detailed abundances are possible to derive from $\mathrm{K}$ and $\mathrm{H}$ band spectra (Lambert et al 1986).

\section{Stellar populations traced by chemical abundances}

Like other spiral galaxies, the Milky Way consists of a bulge, a halo, and a disk. In order to understand the formation and evolution of these components, it is important to know if each of them consists of stars with a common origin (one population) or if they consist of several distinct populations. An important method to detect such sub-populations is based on high-precision abundances, because it is likely that stars of a common origin have about the same chemical composition. In principle, it should be possible to trace the origin of stars through "chemical tagging" (Freeman and Bland-Hawthorn 2002) or methods related to phylogenetic studies (Jofré et al 2017a). 
Before 1950 it was generally believed that all stars had the same universal chemical composition, but from analyses of photographic high-resolution spectra in the 1950ties (Chamberlain and Aller 1951; Baschek 1959; Helfer et al 1959), it was shown that old globular cluster stars and high-velocity stars with halo kinematics have much lower iron-to-hydrogen ratios than the Sun and younger stars. This was interpreted as due to the synthesis of heavy elements by nuclear reactions inside stars (Burbidge et al 1957) and dispersal of the products by mass loss and supernovae explosions increasing the heavy element abundance of the star-forming interstellar gas with time, i.e. chemical evolution. Furthermore, Wallerstein (1962) showed that the abundances of $\mathrm{Mg}, \mathrm{Si}, \mathrm{Ca}$, and $\mathrm{Ti}$ are enhanced relative to the abundance of Fe in metal-poor stars. As these so-called $\alpha$-elements are promptly produced in massive stars and dispersed by Type II SNe, whereas iron is also contributed by Type Ia SNe on a longer timescale, $[\alpha / \mathrm{Fe}]$ at a given $[\mathrm{Fe} / \mathrm{H}]$ depends on the star formation rate in a stellar population (Tinsley 1979; Matteucci and Greggio 1986; Gilmore and Wyse 1998). Therefore, $[\alpha / \mathrm{Fe}]$ is an important label of a stellar population, but other abundance ratios for which the production rates depend on stellar mass and types of supernovae or other sites for nucleosynthesis, notably red giants with mass loss, are also important.

\subsection{Disk populations}

The first major high-precision study of elemental abundances in disk stars was carried out by Edvardsson et al (1993), who selected F and G main-sequence stars in the solar neighbourhood from the catalogue of $u v b y-\beta$ photometry by Olsen (1988) and divided them into nine metallicity bins ranging from $[\mathrm{Fe} / \mathrm{H}] \sim-1.0$ to $\sim$ +0.3 using the Strömgren $m_{1}$ index. In each metallicity bin, high-resolution spectra with $S / N \gtrsim 200$ were obtained for the $\sim 20$ brightest stars and analysed with the EW method. As a main result of the survey, stars in the metallicity range $-0.8<$ $[\mathrm{Fe} / \mathrm{H}]<-0.4$ were found to have differences in $[\alpha / \mathrm{Fe}]$ correlated with their mean orbital distance, $R_{m}$, from the Galactic center. For stars with $R_{m}<7 \mathrm{kpc},[\alpha / \mathrm{Fe}]$ was found to be on average about 0.15 dex higher than $[\alpha / \mathrm{Fe}]$ for stars with $R_{m}>$ $9 \mathrm{kpc}$. Assuming that $R_{m}$ is a measure of the distance from the Galactic center of the stellar birthplace, Edvardsson et al. explained the $[\alpha / \mathrm{Fe}]$ variations as due to a star formation rate that declines with Galactocentric distance; a higher $[\mathrm{Fe} / \mathrm{H}]$ is reached in the inner parts of the Milky Way before Type Ia SNe start contributing iron. This interpretation is, however, in disagreement with the vanishingly small radial gradient in $[\alpha / \mathrm{Fe}]$ found for open disk clusters and Cepheids (see Yong et al 2012; Genovali et al 2015, and references therein).

The variations in $[\alpha / \mathrm{Fe}]$ for metal-poor disk stars were confirmed by independent high-resolution studies of Fuhrmann (1998) and Gratton et al (2000). They interpreted, however, the results as a dichotomy in $[\alpha / \mathrm{Fe}]$ between stars having thin- and thick-disk kinematics 7 . This interpretation was supported by $[\alpha / \mathrm{Fe}]$ values for kinematically selected samples of thin- and thick-disk stars in the solar neighbourhood by Bensby et al (2005) and Reddy et al (2006), but it was unclear

\footnotetext{
7 As shown by Gilmore and Reid (1983), the distribution of stars as a function of distance from the Galactic plane suggests the existence of two distinct components: the thin disk with a scale height of $300 \mathrm{pc}$ corresponding to a velocity dispersion $\sigma(W) \simeq 20 \mathrm{~km} \mathrm{~s}^{-1}$ and the thick disk having a scale height of $1300 \mathrm{pc}$ and $\sigma(W) \simeq 40 \mathrm{~km} \mathrm{~s}^{-1}$.
} 


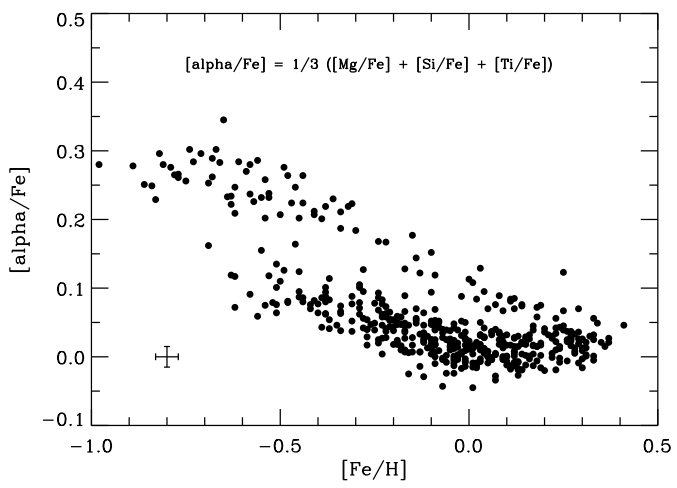

Fig. $4[\alpha / \mathrm{Fe}]$ versus $[\mathrm{Fe} / \mathrm{H}]$ for stars from Adibekvan et al $(2012)$ selected to have $T_{\text {eff }}$ values within a range of $\pm 300 \mathrm{~K}$ from $T_{\text {eff }}$ of the $\mathrm{Sun} .[\mathrm{Ca} / \mathrm{Fe}]$ is not included in the definition of $[\alpha / \mathrm{Fe}]$, because the splitting between the high- and low-alpha sequences is about a factor of two smaller in $[\mathrm{Ca} / \mathrm{Fe}]$ than in $[\mathrm{Mg} / \mathrm{Fe}],[\mathrm{Si} / \mathrm{Fe}]$, and $[\mathrm{Ti} / \mathrm{Fe}]$. Based on data from Adibekyan et al (2012).

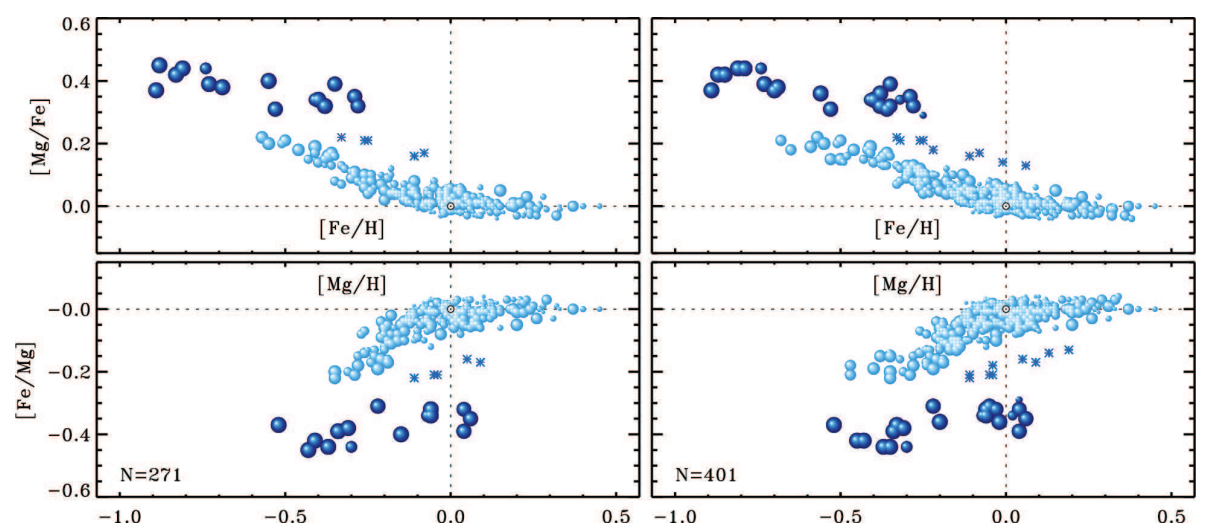

Fig. $5[\mathrm{Mg} / \mathrm{Fe}]$ versus $[\mathrm{Fe} / \mathrm{H}]$ (upper panels) and $[\mathrm{Fe} / \mathrm{Mg}]$ versus $[\mathrm{Mg} / \mathrm{H}]$ (lower panels) for a volume complete sample of $\mathrm{F}$ and $\mathrm{G}$ stars within a distance of $25 \mathrm{pc}$. The left panels refer to a northern sample $\left(\delta>-15^{\circ}\right)$ discussed by Fuhrmann (2011), whereas the right panels show an all-sky survey, where the $d<25$ pc selection is based on the revised Hipparcos distances of van Leeuwen (2007). Stars on the high-Mg sequence are shown with dark blue circles and stars on the low-Mg sequence with light blue circles. The size of the circles is proportional to the stellar age. High-Mg stars have ages around $12 \mathrm{Gyr}$, whereas the ages of low-Mg stars range from 1 to $8 \mathrm{Gyr}$. Stars lying between the high- and low-Mg sequences shown with asterisks have ages around 10 Gyr. Reproduced from Fuhrmann et al (2017) with permission from Klaus Fuhrmann.

from these studies, if stars with $[\alpha / \mathrm{Fe}]$-values between those of the thin and thick disk exist. Clarification has come from two important high-precision studies of samples of nearby stars selected without any kinematical bias. Adibekyan et al (2012) derived abundances of 13 elements from HARPS $R=115000$ spectra of a magnitude limited sample of 1111 FGK main-sequence stars and Fuhrmann et al (2017) ) used $R=50000$ spectra to obtain $\mathrm{Mg}$ and Fe abundances for $\sim 500$ dwarf and subgiant stars with $T_{\text {eff }}>5300 \mathrm{~K}$ within a distance of $25 \mathrm{pc}$. While Adibekyan et al. derived abundances with the EW method, Fuhrmann et al. made spectrum 
synthesis fits to selected lines including Balmer lines for $T_{\text {eff }}$ determination and the Mgb triplet as a constraint on gravity. For both samples, the stars split up into two sequences, high- and low-alpha stars, respectively, clearly separated in $[\alpha / \mathrm{Fe}]$ for the metallicity range $-0.7<[\mathrm{Fe} / \mathrm{H}]<-0.3$ as seen from the diagrams shown in Figs. 4 and [5. A dichotomy is also seen in the $[\mathrm{O} / \mathrm{Fe}]-[\mathrm{Fe} / \mathrm{H}]$ diagram of Bertran de Lis et al (2016, Fig. 1) obtained from APOGEE abundances of K giants in the temperature range $4200<T_{\text {eff }}<4600$.

As suggested by Fuhrmann et al (2017), there may be a distinct population of stars with intermediate values of $[\mathrm{Mg} / \mathrm{Fe}]$ shown by asterisks in Fig. [5. Similar stars lying above the low-alpha sequence in the metallicity range $-0.3<[\mathrm{Fe} / \mathrm{H}]<+0.2$ were found by Adibekyan et al (2012) (see Fig.4) and interpreted by them as a special high-alpha metal-rich ("h $\alpha \mathrm{mr}$ ") population in the solar neighbourhood, perhaps coming from the inner regions of the Milky Way. However, they may just be an extension of the high-alpha sequence as suggested by Bensby et al (2014) based on their $[\mathrm{Ti} / \mathrm{Fe}]-[\mathrm{Fe} / \mathrm{H}]$ diagram of kinematically selected stars in the solar neighbourhood. Such stars are also seen in the Galactic bulge (e.g. Bensby et al 2017). Further studies are needed to clarify the origin of these alpha-enhanced stars in the solar neighbourhood with metallicities around or above solar metallicity.

For the stars in Fig. 5, Fuhrmann et al (2017) have derived ages from evolutionary tracks in the $M_{\text {bol }}-T_{\text {eff }}$ diagram. The high-Mg stars are all very old ( $\left.\sim 12 \mathrm{Gyr}\right)$, whereas the ages of low-Mg stars range from 1 to $8 \mathrm{Gyr}$ and the intermediate stars have ages around $10 \mathrm{Gyr}$. These results agree with ages derived for the Adibekyan et al. sample by Havwood et al (2013), who consider the intermediate stars as an extension of the high-alpha sequence formed in a "thick-disk" 8 phase of the Milky Way with intense star formation lasting about $\sim 4 \mathrm{Gyr}$ and with a downward trend of $[\alpha / \mathrm{Fe}]$ as a function of time due to an increasing contribution of iron from Type Ia SNe. After a quenching of the star formation for a period of $\sim 1 \mathrm{Gyr}$, metal-rich thin-disk stars began to form $\sim 8$ Gyr ago (Snaith et al 2014) leading to a slight decrease of $[\alpha / \mathrm{Fe}]$ with time as also found for solar twins (Nissen 2015; Spina et al 2016a). The metal-poor low-alpha stars are, on the other hand, interpreted as a distinct population born in the outer disk and mixed to the solar neighbourhood by orbital diffusion, whereas the majority of high-alpha stars have been born in the inner disk as suggested from their rotational lag relative to the Local Standard of Rest (LSR). This scenario is supported by in-situ abundances from APOGEE (Hayden et al 2015, Fig. 4); the high-alpha sequence is very pronounced in the inner Galaxy but almost disappears in the outer disk, where the low-alpha sequence dominates.

\subsection{Halo populations}

For a long time it has been discussed if the Galactic halo consists of more than one population of stars. The answer to this question is important for understanding how the Milky Way formed. The monolithic collapse model of Eggen et al (1962) corresponds to a single old population, but from a study of globular clusters Searle and Zinn (1978) suggested that the halo consists of two populations,

8 This designation is somewhat misleading, because the majority of the metal-rich stars on the high-alpha sequence do not have thick-disk kinematics 

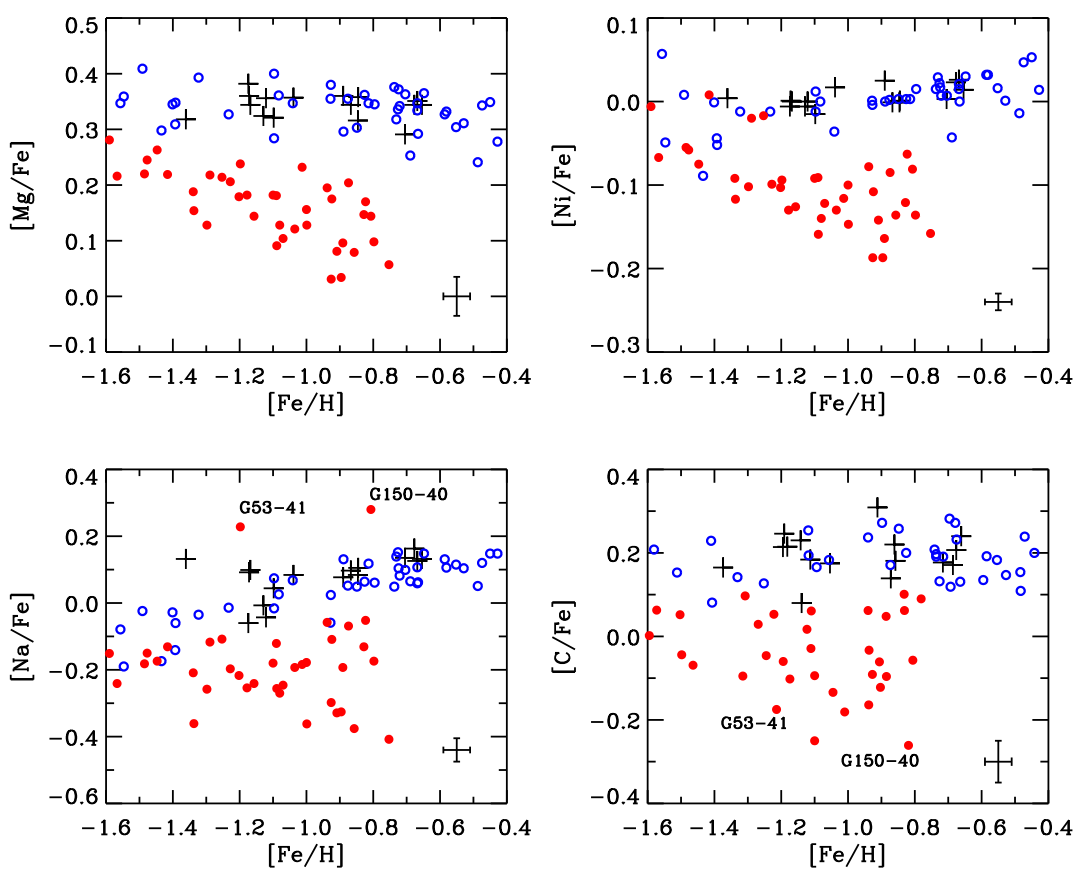

Fig. 6 Abundance ratios versus $[\mathrm{Fe} / \mathrm{H}]$ for the halo and thick-disk stars in Nissen and Schuster (2010). Stars with halo kinematics have been divided into high-alpha stars shown with open blue circles and low-alpha stars shown with filled red circles. Stars with thick-disk kinematics are shown with crosses. Two stars with peculiar high $[\mathrm{Na} / \mathrm{Fe}]$ values are marked. They have low $[\mathrm{C} / \mathrm{Fe}]$ and $[\mathrm{O} / \mathrm{Fe}]$ values and may be born as second generation stars in globular clusters.

i.e. an inner population formed in-situ during a dissipative collapse and an outer spherical population accreted from dwarf galaxies.

Correlations of $[\alpha / \mathrm{Fe}]$ with the kinematics of halo stars have given support to the Searle-Zinn scenario (Nissen and Schuster 1997; Fulbright 2002; Stephens and Boesgaard 2002; Ishigaki et al 2010), but the first clear evidence for the existence of two discrete halo populations was found by Nissen and Schuster (2010, 2011). Based on the $u v b y-\beta$ catalogue of Schuster et al $(2006)$, they selected a nearby $(d<340 \mathrm{pc})$ sample of $94 \mathrm{~F}$ and $\mathrm{G}$ main-sequence stars with $-1.6<[\mathrm{Fe} / \mathrm{H}]<-0.4$ of which the majority have halo kinematics, i.e. a total space velocity with respect to the LSR, $V_{\text {total }}>180 \mathrm{~km} \mathrm{~s}^{-1}$, while 16 stars have thick-disk kinematics. High-resolution, $S / N \sim 200$ spectra were obtained with the ESO VLT/UVES and NOT/FIES spectrographs and used to derive atmospheric parameters and differential abundances of $\mathrm{Na}, \mathrm{Mg}, \mathrm{Si}, \mathrm{Ca}, \mathrm{Ti}, \mathrm{Cr}, \mathrm{Mn}, \mathrm{Fe}, \mathrm{Ni}, \mathrm{Cu}, \mathrm{Zn}, \mathrm{Y}$, and $\mathrm{Ba}$ using the EW method in the LTE approximation. The differential errors of $[\mathrm{X} / \mathrm{Fe}]$ range from 0.01 to 0.04 dex depending on the number and strength of lines available for a given element. In addition, Ramírez et al (2012) and Nissen et al (2014) have derived C and $\mathrm{O}$ abundances for the same sample of stars taking into account non-LTE effects.

As seen from Fig.6, the $[\mathrm{Mg} / \mathrm{Fe}]-[\mathrm{Fe} / \mathrm{H}]$ diagram of Nissen and Schuster (2010) suggests that stars with halo kinematics can be divided into two separate popu- 


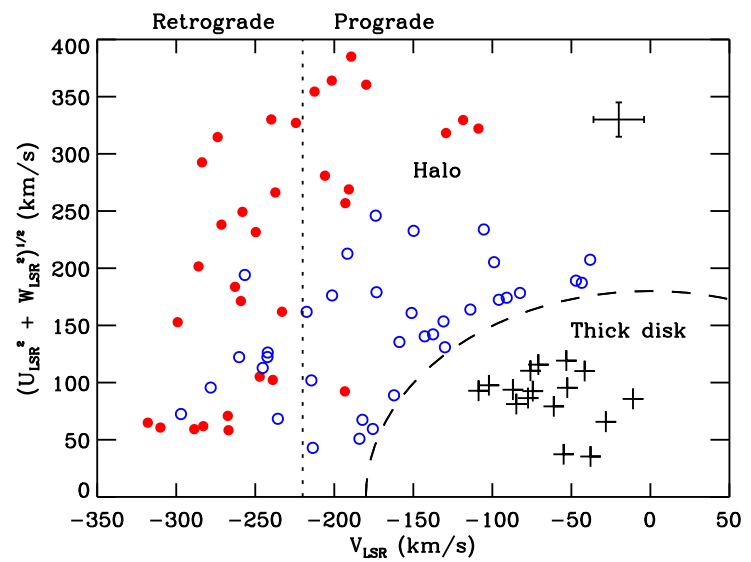

Fig. 7 Toomre diagram for halo and thick-disk stars in Nissen and Schuster (2010) shown with the same symbols as in Fig. [6 Only stars with $[\mathrm{Fe} / \mathrm{H}]>-1.4$ are included. The longdashed line corresponds to $V_{\text {total }}=180 \mathrm{~km} \mathrm{~s}^{-1}$ and the dotted line separates stars moving in retrograde orbits from stars with prograde orbits.

lations, i.e. a high-alpha sequence for which $[\mathrm{Mg} / \mathrm{Fe}]$ lies around a plateau of $\sim 0.35$ dex like the thick-disk stars and a low-alpha sequence for which $[\mathrm{Mg} / \mathrm{Fe}]$ declines with increasing metallicity. The two populations merge at $[\mathrm{Fe} / \mathrm{H}] \simeq-1.6$ but have a separation in $[\mathrm{Mg} / \mathrm{Fe}]$ of about 0.2 dex at $[\mathrm{Fe} / \mathrm{H}]=-0.8$; interestingly, there are no low-alpha stars with $[\mathrm{Fe} / \mathrm{H}]>-0.7$. A separation of the two halo populations is also seen for other abundance ratios such as $[\mathrm{C} / \mathrm{Fe}],[\mathrm{O} / \mathrm{Fe}],[\mathrm{Na} / \mathrm{Fe}]$, $[\mathrm{Si} / \mathrm{Fe}],[\mathrm{Ti} / \mathrm{Fe}],[\mathrm{Ni} / \mathrm{Fe}],[\mathrm{Cu} / \mathrm{Fe}],[\mathrm{Zn} / \mathrm{Fe}]$, and $[\mathrm{Y} / \mathrm{Ba}]$, whereas there is no or very little separation in $[\mathrm{Ca} / \mathrm{Fe}],[\mathrm{Mn} / \mathrm{Fe}]$, and $[\mathrm{Cr} / \mathrm{Fe}]$.

APOGEE abundances of K-giants with $-1.4<[\mathrm{Fe} / \mathrm{H}]-0.7$ and distances reaching beyond the solar neighbourhood confirm the existence of two distinct halo populations (Hawkins et al 2015; Haves et al 2018). In addition to differences in $[\mathrm{C} / \mathrm{Fe}],[\mathrm{O} / \mathrm{Fe}],[\mathrm{Mg} / \mathrm{Fe}]$, and $[\mathrm{Ni} / \mathrm{Fe}]$, the APOGEE data also show a very clear difference in $[\mathrm{Al} / \mathrm{Fe}]$, a ratio which was not explored by Nissen and Schuster (2010).

The trends of $[\alpha / \mathrm{Fe}],[\mathrm{Na} / \mathrm{Fe}],[\mathrm{Ni} / \mathrm{Fe}],[\mathrm{Zn} / \mathrm{Fe}]$, and $[\mathrm{Y} / \mathrm{Ba}]$ as a function of $[\mathrm{Fe} / \mathrm{H}]$ for the low-alpha population resemble the corresponding trends for giant stars in dwarf spheroidal (dSph) galaxies such as Fornax and Sculptor (e.g. Shetrone et al 2001, 2003; Tolstoy et al 2009; Kirby et al 2009; Letarte et al 2010; Skúladóttir et al 2017). This has led to the suggestion that the low-alpha stars originate from dwarf galaxies with relatively low star formation rates, (see the pioneering paper by Matteucci and Brocato 1990), allowing Type Ia SNe to start contributing iron at metallicities already around $[\mathrm{Fe} / \mathrm{H}] \sim-1.6$, whereas highalpha stars have formed in halo regions with such a high star formation rate that Type Ia SNe did not contribute iron until $[\mathrm{Fe} / \mathrm{H}] \sim-0.4$. For a detailed discussion of this scenario and effects of variations in the initial mass function, we refer to McWilliam et al (2013) and Fernández-Alvar et al (2018).

Further evidence that the low-alpha stars belong to an accreted population is obtained from their kinematics. As seen from the Toomre diagram in Fig.7 they tend to move on retrograde orbits and have on average larger $U$ and $W$ veloci- 

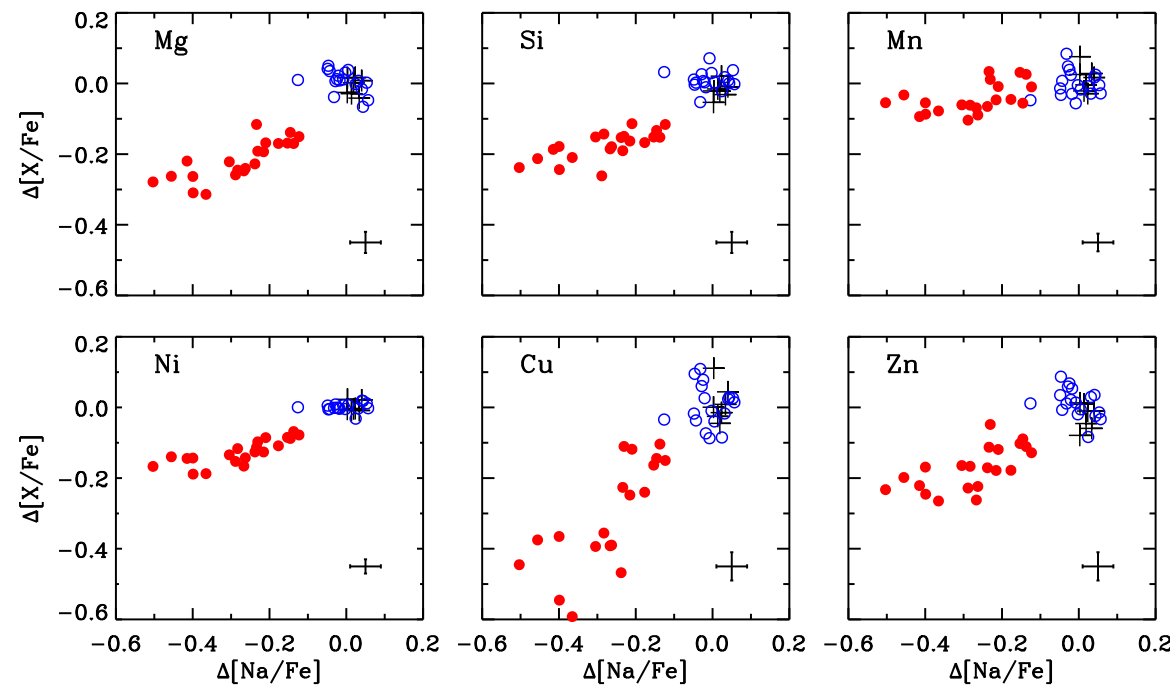

Fig. 8 Correlation between differential abundance ratios for halo and thick-disk stars in Nissen and Schuster (2010, 2011) shown with the same symbols as in Fig.6. Only stars in the metallicity range $-1.1<[\mathrm{Fe} / \mathrm{H}]<-0.7$ are included.

ties than the high-alpha stars. This is confirmed by recent studies of APOGEE abundances in combination with kinematical data from the Gaia DR2 release (Gaia Collaboration et al 2018). As suggested by Havwood et al (2018) and Helmi et al (2018) most of the low-alpha stars may be due to accretion of a single massive satellite galaxy. Evidence of this event is seen as a prominent slightly retrograde structure in the Toomre diagram of $\sim 6000$ high-velocity stars $\left(V_{\text {total }}>210 \mathrm{~km} \mathrm{~s}^{-1}\right)$ with distances less than $1 \mathrm{kpc}$ (Koppelman et al 2018).

As seen from Fig.6, the dispersion of abundance ratios $[\mathrm{X} / \mathrm{Fe}]$ for stars on the low-alpha sequence in the metallicity bin $-1.1<[\mathrm{Fe} / \mathrm{H}]<-0.7$ is a factor 2 - 3 higher than the dispersion for high-alpha and thick-disk stars. This additional dispersion among the low-alpha stars cannot be explained by errors in the abundance ratios; the high- and low-alpha stars have about the same distribution in $T_{\text {eff }}, \log g$, and $[\mathrm{Fe} / \mathrm{H}]$, so non-LTE corrections are expected to change the mean abundances and dispersions for the two populations in a similar way. This has been confirmed in the case of $\mathrm{Cu}$ from a non-LTE analysis of the Nissen and Schuster (2010) spectra by Yan et al (2016).

Figure 8 shows that there is as strong correlation between $\Delta[\mathrm{X} / \mathrm{Fe}]$ (measured relative to the average $[\mathrm{X} / \mathrm{Fe}]$ for the high-alpha population in the $-1.1<$ $[\mathrm{Fe} / \mathrm{H}]<-0.7$ bin) and $\Delta[\mathrm{Na} / \mathrm{Fe}]$, which is the abundance ratio showing the largest variation. Stars in dSph galaxies have more extreme values of $\Delta[\mathrm{X} / \mathrm{Fe}]$, see e.g. the $[\mathrm{Ni} / \mathrm{Fe}]-[\mathrm{Na} / \mathrm{Fe}]$ relation for Fornax by Letarte et al (2010, Fig. 13). This suggests that the low-alpha stars did not form in systems like present-days dSph galaxies, but they may originate from more massive satellite galaxies as proposed by Zolotov et al (2009, 2010). Based on $\Lambda$ CDM modelling of galaxy formation, they predict a dual origin of stellar halos. High-alpha stars were formed in the high-density inner $\sim 1 \mathrm{kpc}$ region but were displaced to the halo by mergers of a few massive satellite galaxies in which the low-alpha stars were born and later 
accreted to the halo. According to this, the dispersion in $[\mathrm{X} / \mathrm{Fe}]$ for the low-alpha population is due to differences in the mass of the satellite galaxies, i.e. a lower mass corresponds to a lower star formation rate or, alternatively, a higher massloss rate from the system, and hence a larger deviation of $[\mathrm{X} / \mathrm{Fe}]$ from the value on the high-alpha sequence. If only a few dwarf galaxies contributed to the lowalpha population, there could be sub-structure in the abundance ratios. A hint of this may possible be seen in the $\Delta[\mathrm{Cu} / \mathrm{Fe}]-\Delta[\mathrm{Na} / \mathrm{Fe}]$ diagram, but a much larger sample of stars with very precise abundances is needed to verify the presence of sub-populations.

\subsection{Extremely metal-poor stars}

The two distinct populations discussed in the previous section have been detected among the more metal-rich halo stars, i.e. in the metallicity range $-1.5<$ $[\mathrm{Fe} / \mathrm{H}]<-0.7$. Obviously, it would be interesting if a similar dichotomy in the distribution of abundance ratios is present for more metal-poor halo stars or if there is a cosmic scatter in abundance ratios at a given metallicity as predicted by some stochastic models (e.g. Argast et al 2000; Karlsson and Gustafsson 2005; Bland-Hawthorn et al 2010) dealing with the chemical enrichment at extremely low metal abundances $([\mathrm{Fe} / \mathrm{H}]<-3.0)$, where only a few supernovae have contributed to the composition of the low-mass stars that can be observed today.

Frebel and Norris (2015) have given a comprehensive review of chemical abundances in extremely metal-poor stars with emphasis on the existence of carbonnormal and carbon-enhanced stars as evidence of two distinct channels of star formation at the earliest times. Here we limit our review to a few works, where the precision of the derived abundances is approaching 0.03 dex.

In the metallicity range $-2.8<[\mathrm{Fe} / \mathrm{H}]<-1.5$, abundances of 18 elements, from Li to Ba, have been obtained by Reggiani et al (2017) for a sample of 23 mainsequence stars selected to have effective temperatures in a range from $6000 \mathrm{~K}$ to $6500 \mathrm{~K}$. VLT/UVES spectra with a resolution of $R \sim 50000$ and $S / N \sim 200$ were analysed with the EW method assuming LTE but adopting non-LTE corrections for NaI, AlI, and CrI. There is no indication of a dichotomy in the distribution of abundance ratios like in the case of more metal-rich halo stars and for the $\alpha$-capture and iron-peak elements the standard deviations of $[\mathrm{X} / \mathrm{Fe}]$ relative to straight line fits as a function of $[\mathrm{Fe} / \mathrm{H}]$ are not significantly larger than the estimated abundance errors, i.e. 0.04 to $0.08 \mathrm{dex}$. This is in agreement with stochastic models predicting negligible cosmic scatter among halo stars with $[\mathrm{Fe} / \mathrm{H}]>-2.5$ due to mixing of products from hundreds of SNe. However, for the neutron-capture elements, Sr, Y, and Ba, there appears to be a significant cosmic scatter on the order of 0.20 dex.

At still lower metallicities, $-4.0<[\mathrm{Fe} / \mathrm{H}]<-2.7$, Cavrel et al (2004) have derived abundances of 17 elements, from $\mathrm{C}$ to $\mathrm{Zn}$, for 35 giant stars of which 22 have $[\mathrm{Fe} / \mathrm{H}]<-3.0$. UVES spectra with a resolution of $R \sim 45000$ and $S / N \sim 100$ to 200 were obtained and analysed with the EW method in LTE but applying 3D corrections for the [O I] $6300 \mathrm{~A}$ line and non-LTE corrections for the resonance lines of $\mathrm{Na}, \mathrm{Al}$, and $\mathrm{K}$. The scatter for most elements around fitted straight lines, $[\mathrm{X} / \mathrm{Fe}]=a[\mathrm{Fe} / \mathrm{H}]+b$, is of the same order as the measurement errors, i.e. 0.05 to 0.10 dex, except for $\mathrm{C}$ and $\mathrm{Na}$. In the case of $[\mathrm{Mg} / \mathrm{Fe}]$, a small 


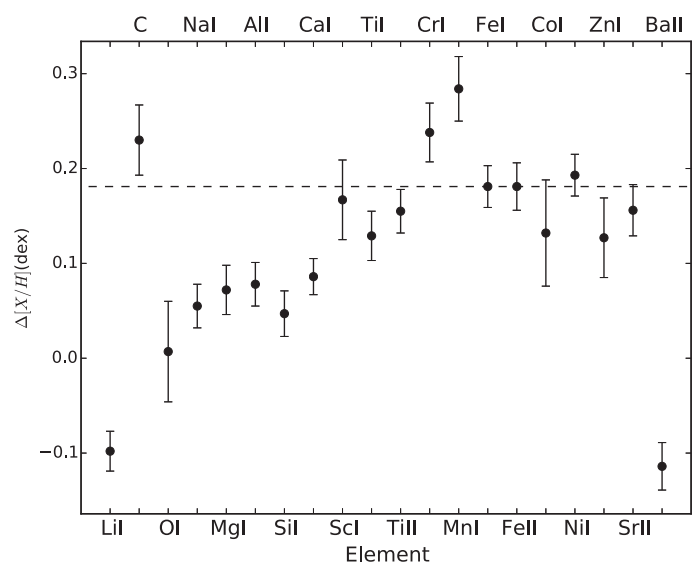

Fig. 9 Differences in abundances between the two extremely metal-poor stars, G 64-37 and G64-12. Reproduced from Reggiani et al (2016) with permission from Henrique Reggiani.

scatter, i.e. $0.06 \mathrm{dex}$, is confirmed in a high-resolution study of 23 main-sequence turnoff stars having $-3.4<[\mathrm{Fe} / \mathrm{H}]<-2.2$ by Arnone et al $(2005)$. These results do not agree with the stochastic chemical enrichment models of Argast et al (2000) and Karlsson and Gustafsson (2005), who predict $\sigma[\mathrm{Mg} / \mathrm{Fe}] \sim 0.2$ to 0.4 dex (depending on the adopted yield of core-collaps $\mathrm{SNe}$ ) for the metallicity range $-4.0<[\mathrm{Fe} / \mathrm{H}]<-3.0$.

In contrast to the small scatter of $[\mathrm{X} / \mathrm{Fe}]$ for the $\alpha$-capture and iron-peak elements, there is a large scatter for the neutron-capture elements among stars with $[\mathrm{Fe} / \mathrm{H}] \lesssim-3.0$. For the Cayrel et al (2004) sample of K giants, François et al (2007) find a scatter of 0.6 dex for $[\mathrm{Sr} / \mathrm{Fe}]$ and for $\mathrm{Y}, \mathrm{Zr}$, and $\mathrm{Ba}$ the scatter of $[\mathrm{X} / \mathrm{Fe}]$ is $\sim 0.4$ dex, i.e. significantly greater than the observational errors of $\sigma[\mathrm{X} / \mathrm{Fe}] \sim 0.1$ dex. Also for heavier elements like Eu, Gd, Dy, and Er there is a cosmic scatter of $[\mathrm{X} / \mathrm{Fe}]$. As discussed by e.g. Cescutti et al (2015), this may be explained by stochastic chemical evolution models with contributions to the $r$-process from both core-collapse $\mathrm{SNe}$ and merging neutron stars. In view of the gravitational-wave detection of two merging neutron stars by LIGO/Virgo (Abbott et al 2017) and the likely production of $r$-process elements in connection with this event (e.g. Côté et al 2018), it would be interesting to obtain highprecision abundances of neutron-capture elements in a large sample of extremely metal-poor stars to test the relative importance of neutron-star mergers and corecollapse $\mathrm{SNe}$ as sites for the $r$-process.

As discussed by Karlsson and Gustafsson (2005) there is special reason to obtain high-precision abundances for very metal-poor halo stars, namely to look for narrow Single Supernovae Sequences (SSSs) in various abundance diagrams, e.g. $[\mathrm{Ti} / \mathrm{Mg}]$ versus $[\mathrm{Ca} / \mathrm{Mg}]$, representing the location of stars with major contributions from single SNe of different masses. If such SSSs were detected one would get direct information about first generation supernovae yields. The prediction of these sequences is based on the assumption that star formation is unclustered and that stars are randomly distributed in space. If instead long-lived stars in the halo are primarily formed in massive clusters with a homogeneous composi- 
tion, one should see tight groups of stars in abundance space at $[\mathrm{Fe} / \mathrm{H}]<-3.0$ (Bland-Hawthorn et al 2010). In both cases the challenge will be to obtain abundances with precisions better than 0.05 dex for hundreds of very metal-poor stars.

Reggiani et al (2016) have shown that it is indeed possible to reach precisions of $\sigma[\mathrm{X} / \mathrm{Fe}]<0.05$ dex for the relative abundances of very metal-poor stars. Spectra with $R \sim 95000$ and $S / N \sim 800$ for two main-sequence turnoff stars (G 64-12 and G 64-37 with respectively $[\mathrm{Fe} / \mathrm{H}]=-3.2$ and -3.0$)$ were obtained with HIRES at the Keck $10 \mathrm{~m}$ telescope and analysed in a strictly (line-by-line) differential way assuming LTE. Interesting differences of $[\mathrm{X} / \mathrm{Fe}]$ between the two stars have been found (see Fig. 9) suggesting that SNe of different masses have enriched the gas clouds out of which the stars were formed. Given that G 64-12 and G64-37 $(V=11.45$ and $V=11.15)$ are among the brightest stars having $[\mathrm{Fe} / \mathrm{H}]<-3.0$, one probably needs observing time at extremely large telescopes to obtain spectra with similar high quality for hundreds of extremely metal-poor stars,

\subsection{Bulge populations}

It has been much discussed whether the Milky Way has a "classical" bulge consisting of old stars formed rapidly during an initial dissipative collapse phase or if it is a "pseudo-bulge" formed over a longer period due to dynamical instabilities in the Galactic disk. Stellar abundances may help to answer this question by providing information on the timescale for the formation of the bulge.

Beginning with the pioneering model-atmosphere analysis of high resolution spectra of K giants in the Galactic bulge by McWilliam and Rich (1994), there have been many studies of the $[\alpha / \mathrm{Fe}]-[\mathrm{Fe} / \mathrm{H}]$ trends with conflicting results (see review by McWilliam 2016). In some works a high plateau of $[\alpha / \mathrm{Fe}] \sim 0.3$ extends to solar metallicities corresponding to a very high star formation rate as predicted for models of a classical bulge. In other works $[\alpha / \mathrm{Fe}]$ starts to decline at $[\mathrm{Fe} / \mathrm{H}] \sim-0.5$ like in the thick disk supporting the pseudo-bulge scenario. These differences are probably due to statistical and systematical errors of $[\mathrm{Fe} / \mathrm{H}]$ and $[\alpha / \mathrm{Fe}]$; as shown by Schultheis et al (2017) there are differences on the order of 0.10 to 0.15 dex between previously published abundances and new abundances derived from APOGEE spectra.

The median $[\mathrm{Mg} / \mathrm{Fe}]-[\mathrm{Fe} / \mathrm{H}]$ relation based on APOGEE abundances for $\sim 300$ bulge giants in Baade's window (Schultheis et al 2017, Fig. 11) is nearly the same as the relation for local thick-disk stars, but there is a large scatter in $[\mathrm{Mg} / \mathrm{Fe}]$ at a given $[\mathrm{Fe} / \mathrm{H}]$; for stars with $[\mathrm{Fe} / \mathrm{H}]>0.0,[\mathrm{Mg} / \mathrm{Fe}]$ ranges from -0.05 to +0.25 dex. It is unclear if this scatter is cosmic or due to errors in $[\mathrm{Mg} / \mathrm{Fe}]$. A more tight $[\mathrm{Mg} / \mathrm{Fe}]-[\mathrm{Fe} / \mathrm{H}]$ relation was found for $\sim 2000$ red clump giants in 11 bulge fields by Roias-Arriagada et al (2017), who used $R \simeq 16000, S / N>80$ spectra of the 8480-9000 $\AA$ region from the Gaia-ESO survey to derive atmospheric parameters and abundances. Except for a few outliers with low $[\alpha / \mathrm{Fe}]$ values (Recio-Blanco et al 2017), the $[\mathrm{Mg} / \mathrm{Fe}]-[\mathrm{Fe} / \mathrm{H}]$ relation agrees well with that of local thick-disk stars. Interestingly, the metallicity distribution of the bulge stars is bimodal with peaks at $[\mathrm{Fe} / \mathrm{H}] \sim-0.4$ and $[\mathrm{Fe} / \mathrm{H}] \sim+0.3$ and with the metal-rich population claimed to reach $[\mathrm{Fe} / \mathrm{H}]$ values as high as $+0.7 \mathrm{dex}$. Based on the kinematics as determined from radial velocities, Rojas-Arriagada et al (2017) associate the metal-rich population with a boxy/peanut bulge formed in the early 


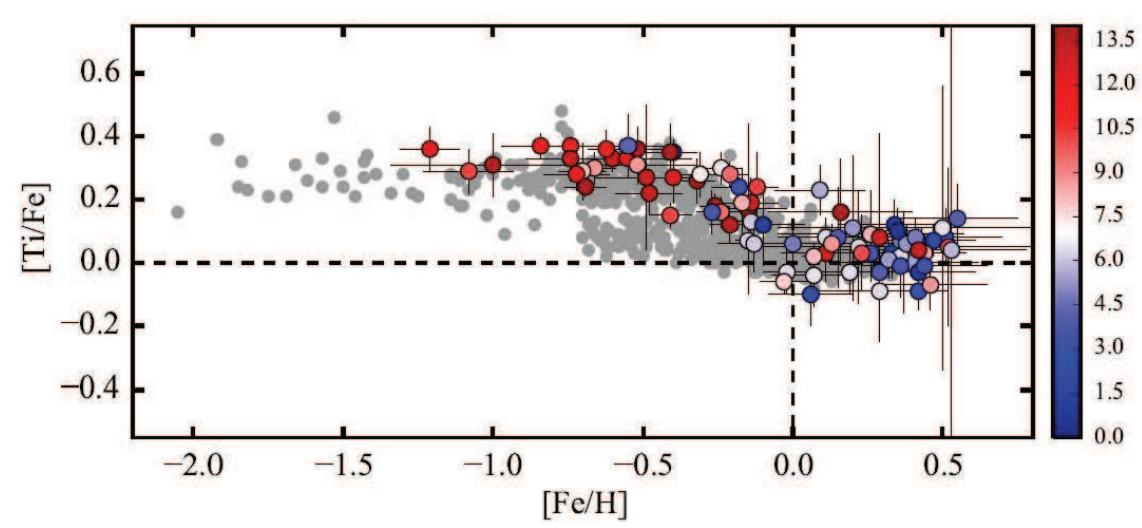

Fig. $10[\mathrm{Ti} / \mathrm{Fe}]$ versus $[\mathrm{Fe} / \mathrm{H}]$ for microlensed dwarf stars in the Galactic bulge. The colours show isochronic ages in Gyr as given in the bar on the right-hand side. Grey circles are the solar neighbourhood dwarf stars from Bensby et al (2014). Reproduced from Bensby et al (2017) with permission from Thomas Bensby.

thin disk and the metal-poor population with a classical bulge, although it could not be ruled out that it is due to a secular evolution of the early thick disk.

During the last decade, Bensby et al (2017) have carried out a unique study of the Galactic bulge by determining abundances and ages of microlensed dwarf and subgiant stars. Normally such stars in the bulge have apparent $V$ magnitudes in the range 18 - 20, but during a microlensing event they brighten to $V=13-16$ for a few hours. This made it possible to obtain high-resolution spectra $(R \sim 40000)$ with S/N up to 200 for 90 stars primarily with the UVES spectrograph at the ESO VLT. The spectra were analysed with the EW method in the same way as spectra of thin- and thick-disk stars in the solar neigbourhood (Bensby et al 2014). Abundances of 11 elements (Na, Mg, Al, Si, Ca, Ti, Cr, Fe, Zn, Y, and $\mathrm{Ba})$ were determined with errors of $[\mathrm{X} / \mathrm{Fe}]$ typically in the range $0.05-0.10 \mathrm{dex}$. Furthermore, ages could be estimated from isochrones in the $\log g-T_{\text {eff }}$ diagram.

The $[\mathrm{X} / \mathrm{Fe}]-[\mathrm{Fe} / \mathrm{H}]$ relations of the microlensed stars follow the trends of local thick-disk stars, but with a $\sim 0.10$ dex higher $[\mathrm{Fe} / \mathrm{H}]$ of the "knee", where $[\alpha / \mathrm{Fe}]$ starts to decline from the plateau value, see Fig. 10. There are indications of several peaks in the $[\mathrm{Fe} / \mathrm{H}]$ and age distributions and about $35 \%$ of the bulge stars with $[\mathrm{Fe} / \mathrm{H}]>0$ have ages younger than $8 \mathrm{Gyr}$. This seems at odds with colour-magnitude diagrams suggesting that all bulge stars are very old. However, as pointed out by Bensby et al., old metal-poor and young metal-rich isochrones are nearly indistinguishable from each other. Altogether, the results of Bensby et al. favor a secular origin of the Galactic bulge, but a minor classical component cannot be excluded.

Further improvements of the precision of abundance determinations for bulge stars to a level of $\sim 0.03$ dex would be important to obtain more information about multiple populations in the Galactic bulge. Possibilities of using infrared spectra of late $\mathrm{K}$ and $\mathrm{M}$ giants for this purpose were discussed in Sect. 2.7. 


\subsection{Chemical tagging and phylogenetic studies}

The idea of "chemical tagging" is to use stellar abundances to identify stars now widely dispersed in space as born in the same molecular cloud. It is based on the assumption that stars in a given cluster have the same chemical composition at birth and that changes in the chemical composition of stellar atmospheres over time due to e.g. atomic diffusion are sufficiently similar for all stars studied. If these assumptions are adequate, chemical tagging has the potential of providing interesting information on the accretion and star-formation history of the Galaxy. Several large on-going abundance surveys such as APOGEE (Majewski et al 2017), GaiaESO (Gilmore et al 2012), and GALAH (Bland-Hawthorn and Sharma 2016) were designed with this purpose in mind.

A thorough discussion of the prospects of chemical tagging has been presented by Ting et al (2015). Important parameters for the method is the dimensionality of chemical space, i.e. the number of abundance ratios varying independently, and the amplitude of variations relative to the precision of the measurements. This determines the number of chemical cells (i.e. the number of distinguishable cloudto-cloud variations in chemical space), which together with the number of stars in the survey is critical for success of the method. Simulations for the GALAH survey show that with a sample of $\sim 10^{6}$ stars and a number of chemical cells, $N_{\text {cells }} \gtrsim 5 \times$ $10^{4}$, it may be possible to detect disrupted clusters with masses $M \gtrsim 10^{5} M \odot$. This assumes that the clusters are evenly distributed over chemical space, which may not be the case. Interestingly, Ting et al. show that an improvement of the precision $(\sigma)$ of the derived abundances is much more efficient than increasing the size of the sample, because $N_{\text {cells }} \propto \sigma^{-N_{\text {dim }}}$, where $N_{\text {dim }}$ is the dimensionality of chemical space. With $N_{\text {dim }}=8$, as estimated for the GALAH survey, a decrease of $\sigma$ by a factor of two would increase $N_{\text {cells }}$ with a factor of 250 !

In an application of chemical tagging on a sample of $\sim 10^{5}$ stars (including known clusters) with APOGEE abundances for 15 elements, Hogg et al (2016) were able to detect two globular clusters (M 5 and M 13), the Sagittarius stream, a halo structure with high velocity dispersion, and a thin-disk structure possibly associated with the Milky Way bar. This shows that chemical tagging can work on high-mass structures especially at low metallicities. However, none of the known open cluster clusters were detected. In a test of chemical tagging of about 200 stars in 31 open clusters with abundances of 14 elements determined from HARPS and UVES spectra, Blanco-Cuaresma et al (2015) also found that clusters could not be chemically recovered due to overlap in chemical space. Furthermore, although Mitschang et al (2014) in a chemical tagging experiment on a sample of $\sim 700$ stars with abundances of 12 elements from Bensby et al (2014) found many groups, they seem to represent coeval associations of stars instead of stars formed from individual molecular clouds implying a homogeneous chemical evolution in the Milky Way. This suggests that it will be difficult to determine the details of the star formation history of the disk from chemical tagging. The abundance precision in the mentioned studies is on the order of $0.03-0.06$ dex. Improving the precisions to 0.01-0.02 dex will enhance the potential of the method greatly as discussed above. However, one may then run into problems with the assumption of chemical homogeneity of stars born in a given molecular cloud related to dust-gas separation effects on the composition of stars (see Sect. 4), and one has to limit the sample of stars to a small region of the $T_{\text {eff }}-\log g$ diagram to ensure that atomic diffusion 
of elements modify the atmospheric composition for all stars born in a given cloud in the same way (Dotter et al 2017).

A new way of using chemical abundances to study the chemical evolution of stellar populations has been proposed by Jofré et al (2017a), i.e. to apply phylogenetic techniques from biology on chemical abundances (as "a stellar DNA") to construct evolutionary trees. As an example, the method was used for a sample of 22 solar twins (including the Sun), for which Nissen (2015, 2016) has derived very precise differential $(\sigma \sim 0.01 \mathrm{dex})[\mathrm{X} / \mathrm{Fe}]$ abundance ratios for 17 elements representing the main nucleosynthesis channels, i.e. $\alpha$-capture $(\mathrm{C}, \mathrm{O}, \mathrm{Mg}, \mathrm{Si}, \mathrm{S}, \mathrm{Ca}$, $\mathrm{Ti}$ ), odd- $Z$ processes ( $\mathrm{Na}, \mathrm{Al}, \mathrm{Sc}$ ), iron-peak processes ( $\mathrm{Cr}, \mathrm{Mn}, \mathrm{Ni}, \mathrm{Cu}, \mathrm{Zn}$ ), and neutron capture $(\mathrm{Y}, \mathrm{Ba})$. Based on "chemical distances" for pairs of stars an evolutionary tree with three branches was defined, but six stars could not be assigned to any branch with statistical significance. From isochronic ages and kinematics of the stars, the three branches can be identified with an old (9-10 Gyr) thick-disk population, a relatively young $(1-5 \mathrm{Gyr})$ thin-disk population and an intermediate population that appears discrete in several abundance ratios and may be related to the intermediate population suggested by Fuhrmann et al (2017). Compared to traditional methods of detecting stellar populations from clustering in various abundance diagrams, the phylogenetic method may add information on the sequence of stars born in a given population. Hence, the rate of chemical evolution for a given population can be determined by comparing chemical distances with stellar ages. A basic assumption inherent in the method is that the sequence of populations in the explored region of the Galaxy has followed a continuous track with essentially no separate and distinctively different contributions, from e.g. infalling sub-systems or wide migration. Yet, the method seems promising and it would be interesting to see how it works for large samples of stars having abundance precisions on the order of 0.03 dex, e.g. the APOGEE K giants.

\section{The Sun compared to solar twins}

The concept of "solar twins" was early discussed (Cavrel de Strobel et al 1981; Cavrel de Strobel 1996) and used in defining a standard solar proxy for colour calibrations. Meléndez et al (2009) defined a sample of 11 twins, based on the Hipparcos catalogue, with maximum departures of $75 \mathrm{~K}$ in effective temperature, of 0.10 dex in logarithmic surface gravity and of 0.07 dex in $[\mathrm{Fe} / \mathrm{H}]$ from the corresponding solar values. Spectra were obtained with a wavelength resolution of $R=65000$ and a $S / N \sim 450$ per pixel. Using a selected set of spectral lines and an analysis as model-independent as possible, abundances were derived with the individual line EW method relative to the Sun (as reflected in asteroid spectra). The resulting abundances for the individual stars were found to have intrinsic errors on the order of $0.01 \mathrm{dex}$, and significant differences between the stellar average logarithmic abundance ratio $[\mathrm{X} / \mathrm{Fe}]$ for each element $\mathrm{X}$ and the corresponding solar abundance were found. The difference have a range of about 0.08 dex and clearly correlate with the condensation temperature $T_{c}$ for the element in a gas with solar composition, as listed by Lodders (2003) (see Fig. 11). Bedell et al (2018) have verified this tendency for a sample of 79 solar twins and also show that the Sun is not an isolated outlier but a star in the tail of a distribution of the ratio $R$ of abundances of "refractories" (high $T_{c}$ ) relative to "volatiles" (low $T_{c}$ ). 


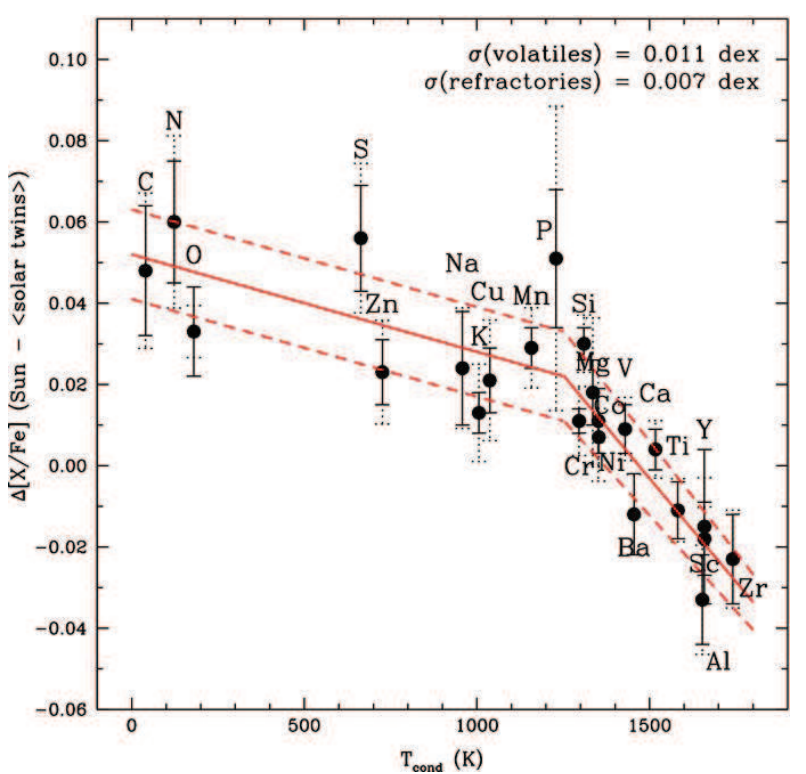

Fig. 11 Differences in $[\mathrm{X} / \mathrm{Fe}]$ between the Sun and the mean values for 11 solar twins as a function of elemental condensation temperature. The solid red lines show a double linear fit to the data broken at $T_{\mathrm{c}}=1200 \mathrm{~K}$, while the dashed lines represent the standard deviation from the fits. The 1-sigma errors in the mean abundances of the solar twins are shown with solid error bars, while the dotted error bars show the 1-sigma errors of the relative abundances (including errors in both the Sun and the twins). Reproduced from Meléndez et al (2009) with permission from Jorge Meléndez.

On the order of $5 \%$ of the twins in the solar neighbourhood are judged to have ratios $R$ close to the solar ratio or less. An interesting example of a star which is a solar twin, also in view of its low $R$ ratio, is M67-1194, in the old rich cluster M67 (Önehag et al 2011; Liu et al 2016a). Also our nearest solar-analogue star, $\alpha$ Cen A, has a $[\mathrm{X} / \mathrm{Fe}]-T_{\mathrm{c}}$ trend similar to that of the Sun (Morel 2018).

A number of possible explanations for this "Meléndez effect" have been considered. The possibility that the stellar spectra are different from the solar one just because we observe the Sun at a special angle, relative to its axis of rotation, could be clearly refuted by solar observations at different disk locations (Kiselman et al 2011). Remaining alternatives included effects of Galactic evolution and nucleosynthesis, the cleansing of the pre-solar cloud from dust by radiation pressure from hot stars or by the Sun itself, the late accretion of the proto-planetary disk cleansed from refractories in dust by planet formation, the results of planetary engulfment to various degrees for different stars, and the thermal processing and outflows from the inner accretion disk.

It has been discussed whether the anomaly of the Sun would disappear if the age dependences of $[\mathrm{X} / \mathrm{Fe}]$ for the solar twins were taken into account (Adibekyan et al 2014), but Bedell et al (2018) have convincingly shown this not to be the case. The idea that the pre-solar cloud was cleansed by the radiation from hot stars was inspired by the possibility that the solar birth cloud was early on polluted by at least one core-collapse supernova, which then implies a probable solar origin in a fairly rich cluster (Adams 2010). This led to a spectroscopic study of the solar-like 
stars in the rich and old open cluster Messier 67 (Önehag et al 2011, 2014), which indeed were found to have abundances more close to the Sun than most solar twins, supporting the hypothesis. The $\mathrm{S} / \mathrm{N}$ of the spectra of these apparently faint stars were, however, not comparable to those of the nearby twins, and further observations are warranted. Also, Gustafsson (2018a), found by model calculations that the mechanism would not be effective enough to cleanse most of the gas forming a full cluster from dust, and that turbulence in the cloud would probably strongly reduce the degree of dust-cleansing as a whole. Another possibility studied by Gustafsson (2018b) is that the radiation from the proto-Sun itself partially cleansed the gas to be accreted in the late stages of the star formation process. For this mechanism to work, one has to assume a relatively slow accretion rate (on the order of $0.01 \mathrm{M}_{\odot} / \mathrm{Myr}$ ), active during at least $1 \mathrm{Myr}$, at a stage so late that the solar convection zone has retracted toward the surface enough to contain only a few percent of the solar mass.

A similar constraint related to the time scale of deep convection (suggested by pre-main- sequence standard models to last for about $30 \mathrm{Myr}$ ) is also necessary if the cleansing of the proto-planetary disk by planet formation is taken as the explanation for the effect. In this case, the life-time of the gas disk, assumed to be finally accreted to the star, has to be prolonged considerably beyond the few million years usually suggested (e.g. Wyatt 2008). Another way of solving this dilemma is to postulate a much more rapid retraction of the convection zone to the surface, due to episodic accretion onto the star, following Baraffe and Chabrier (2010).

Yet another way to explain the Meléndez effect is to assume different frequencies of engulfed planets for different stars (Melendez and Ramirez 2016; Kunitomo et al 2018), in which case the Sun would have had comparatively few accreted planets, or differences in the time when engulfment occurs, in which case the Sun would have had comparatively early such events when the convection zone was still deep, so that the swallowed planet(s) did not affect the solar surface composition very much. An attractive feature with this explanation is that it would explain an interesting observation by Gonzalez et al (2010), namely that the meteorites of chondrite type CI, which are supposed to show the original chemical composition of the original solar cloud, tend to have even lower refractory/volatile ratio than the Sun itself. The "self-cleansing hypothesis" mentioned above would however, also suggest a natural explanation for this: the amount of accreted cleansed gas (then presumably setting the final composition of the disk and its chondrites) may be smaller than the mass of the convective zone of the star.

The different hypotheses concerning the origin of the Meléndez effect demonstrate how accurate studies of abundance ratios may lead to new clues to important but enigmatic cosmogonic processes. However, as noted in Sect. 3.5, the phenomenon also leads to some questioning concerning the possibility of chemical tagging as a means of detailing the history of Galactic evolution. 


\section{Abundance differences in binaries and clusters}

\subsection{Binaries}

Since two decades abundance differences between components in binary stars have been searched for (Gratton et al 2001a), and possible findings have been tentatively related to differences in the planetary systems of the components. These efforts have been concentrated towards wide binaries with almost identical components in terms of effective temperature and surface gravity, indeed excellent targets for differential spectroscopy in order to exploit the high precision achievable in a differential analysis. One good example of such a system is $16 \mathrm{Cyg} \mathrm{A}$ and B, both stars with similar parameters close to solar, the A component being $60-80$ $\mathrm{K}$ hotter than the $\mathrm{B}$ component. $\mathrm{B}$ was found to have a planet more massive than Jupiter by Cochran et al (1997), while no planet has as yet been found around A. Gonzalez (1998) and Laws and Gonzalez (2001) found the A component to be slightly more iron rich than the B component (by 0.05 and 0.025 dex, respectively) and Laws and Gonzalez (2001) ascribed this difference to planet formation and even traced a tendency for the abundance difference with condensation temperature. Ramírez et al (2011) also found the A component to be more metal-rich than the B component, by $0.04 \mathrm{dex} \pm 0.01 \mathrm{dex}$, but did not trace any significant difference in this respect between refractories and volatiles. Tucci Maia et al (2014), however, concluded that the volatiles depart by 0.03 dex at a mean in between the components and found the refractories to show differences that grow with condensation temperature for the element. This was confirmed by Nissen et al (2017). The total amplitude in the variation $\Delta[\mathrm{X} / \mathrm{H}]$ between the components is 0.02 dex. The authors estimate the core of the rocky planet, which is supposed to bind the refractories that would else have ended in the convection zone of the B component, to be of the same order of magnitude as the core of Jupiter.

Another interesting example is the binary XO-2, consisting of two very similar $\mathrm{K}$ dwarfs with estimated effective temperatures around $5300 \mathrm{~K}$ and masses of about 0.97 solar masses (Biazzo et al 2015; Ramírez et al 2015). Both stars have planetary systems. In this case, the amplitude in $\Delta[\mathrm{X} / \mathrm{H}]$ extends over 0.10 dex (see Fig. 12), which is much since the low effective temperatures indicate that the mass of the convective zone is much greater than higher up along the main sequence. Therefore, a difference of about 50 Earth masses of refractory material in between the stars is needed to affect so much mass sufficiently and explain the differences. It should be noted that the late spectral type of these stars makes the abundance analysis more difficult due to the rich and blended spectra (Teske et al 2015). Internal errors in the abundance differences between the stars vary between 0.02 dex for some elements up to 0.05 dex or even more.

Yet another interesting binary is WASP-94 A and B. These dwarf stars are more metal rich and hotter than the Sun, of spectral types F8 and F9, and both stars host a hot Jupiter-like planet. According to Teske et al (2016) abundance differences between the $\mathrm{A}$ and $\mathrm{B}$ components show a unique pattern compared with those of the other binaries known to show different abundances: A is poorer in volatiles than B by 0.02 dex but richer than B in refractories by 0.01 dex. Although these effects are small, they are still significant, at least if the internal precision in the abundance differences claimed by the authors to be about 0.005 dex is adopted as realistic errors. 


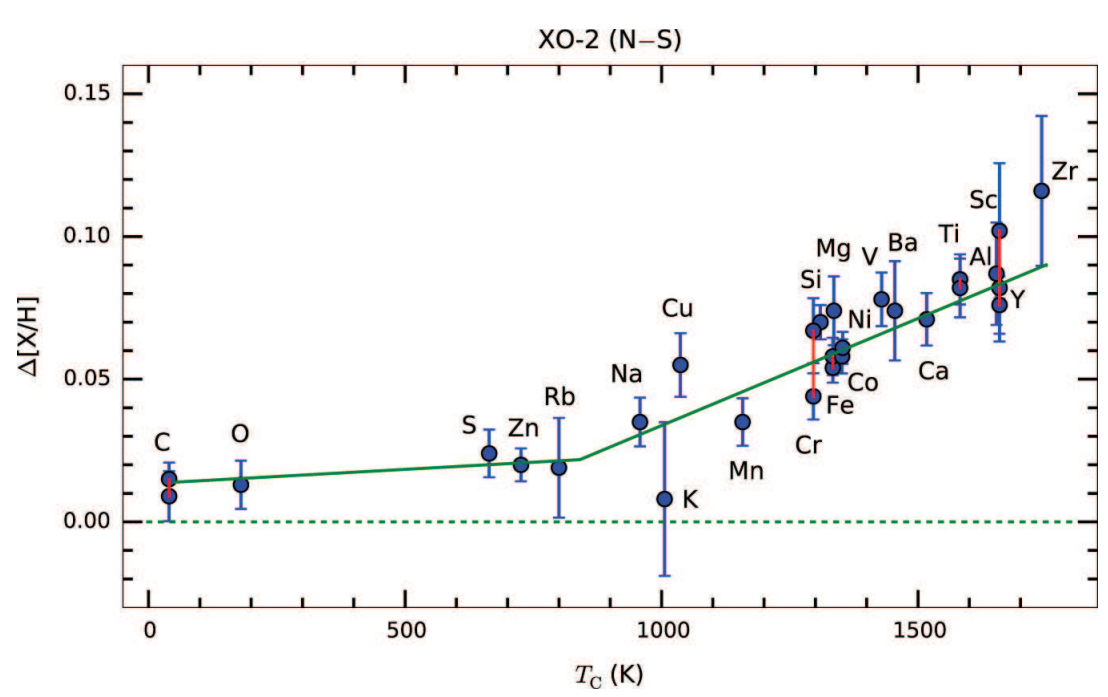

Fig. 12 The abundance difference between the binary stars $\mathrm{XO}-2 \mathrm{~N}$ and $\mathrm{XO}-2 \mathrm{~S}$ as a function of the elements' condensation temperature. The solid green line is a double linear fit to the data broken at $T_{\mathrm{c}}=840 \mathrm{~K}$. Red vertical lines connect $[\mathrm{X} / \mathrm{H}]$-values derived from two species of the same element, e.g. Cr I and Cr II. Reproduced from Ramírez et al (2015) with permission from Ivan Ramírez.

The two components in the binary HAT-P-4 were found by Saffe et al (2017) to be somewhat hotter (by $+250 \mathrm{~K}$ ) and more metal-rich than the Sun (by up to $0.3 \mathrm{dex}$ ). The A component has a hot Jupiter in orbit while no planet has been found around B. In a detailed line-by-line differential analysis of spectra with high $\mathrm{S} / \mathrm{N}$, Saffe et al (2017) found the A component to be significantly richer in refractory elements than $\mathrm{B}$ by as much as about 0.1 dex (one of the greatest internal differences as yet found among binary abundances), while the stars have more similar abundances in volatiles. Another noteworthy effect is that A has a significantly higher Li abundance, contrary to the suggestion by Delgado Mena et al (2014) that close planets cause more efficient Li depletion. A scenario involving formation and later infall of rocky planets and planetesimals onto the A component, while the giant planet spirals inwards, is proposed by the authors.

Another quite interesting wide binary is the two co-moving solar-like stars HD240430 and HD240429, explored by Oh et al (2018). The authors find, again in a study at high resolution and a S/N of about 200, that the HD240430 has roughly solar-like volatile abundances while its refractories are enhanced by about 0.2 dex with respect to the Sun and its companion (a true record holder in this respect). HD 240429 has both volatiles and refractories close to solar. We also note that HD240430 has a significantly higher Li abundance, and that none of the stars is known to have a planet today.

One should not be misled by the selection of examples above to believe that all solar-type binary stars show pronounced abundance differences scaling with condensation temperatures. One example of the contrary is the wide system HD80606/ 80607 where the two components were found in a high-precision analysis by Liu et al (2018) to depart in metallicity by $0.013 \pm 0.002$ dex (!) but with no clear variation with condensation temperature. 
The results for binary compositions clearly demonstrate the possibilities of strictly differential abundance analysis to trace interesting effects of fractionation of chemical elements in the early evolution of stars and planetary systems. They again suggest that one should not overemphasize the possibilities of chemical tagging in the study of Galactic evolution. A useful summary of the results achieved in abundances studies for wide binaries is provided in an appendix by Oh et al (2018).

\subsection{Clusters}

Since long, the chemical homogeneity of star clusters has been discussed. Chemical differences have been found among stars in the same cluster and may be consequences of stellar evolution processes, such as dredge-up of processed matter due to CNO burning (Kraft 1994), or destruction of Li (Boesgaard and Tripicco 1986) and Be (Smilianic et al 2010), or of "diffusion" - or sooner the interaction of radiative pressure, turbulent and other mixing, and sinking in the gravitational field (Korn et al 2007; Nordlander et al 2012; Gruyters et al 2014; Bertelli Motta et al 2018). It seems clear that the effects found are not to be referred to departures from LTE; differences in atmospheric structure should, however, be further explored. The study of these processes in clusters involves comparison of stars in different evolutionary stages, i.e. with different parameters which, although the results of the studies may be truly interesting, makes it difficult to achieve the degree of accuracy discussed here. In the diffusion studies, this technical difficulty may be partly circumvented by comparison of stars with very similar effective temperatures, although on different branches in the colour-magnitude diagram, such as the main-sequence and subgiant-giant branches, where the effects of diffusion are expected to be different. Of a special interest is, however, inter-comparison between very similar stars - abundance differences between these could indicate inhomogeneities in the gas cloud in which the stars were formed. Such differences would be contrary to the expectation that the proto-cluster would be homogenized before the first supernova (Krumholz and Matzner 2009) and have important bearings on the cluster- and star-formation processes. Also, such findings would give fundamental constraints on the possibilities of studying Galactic evolution by "chemical tagging".

For the globular clusters, star-to-star variations with increased $\mathrm{He}, \mathrm{N}$ and $\mathrm{Na}$, and correspondingly decreased $\mathrm{C}$ and $\mathrm{O}$ abundances have been known and studied since long. Abundance variations of $\mathrm{C}$ and $\mathrm{N}$ for stars of similar luminosity on the giant branch were traced by Bell and Dickens (1980) and clear evidence of a Na-O anti-correlation was found by Kraft et al (1993) for giants in M 13 and by Gratton et al (2001b) for turnoff stars in NGC 6752. The effects were interpreted as signatures of hot $\mathrm{H}$ burning and effects of multiple generations of stars. Carretta et al (2009) published homogeneous $\mathrm{O}, \mathrm{Na}, \mathrm{Mg}, \mathrm{Al}$ and $\mathrm{Si}$ abundances based on high-resolution spectra for more than 200 red giants in 17 Galactic globular clusters with widely different over-all metallicities. These authors found starto-star variations in $\mathrm{O}$ and $\mathrm{Na}$ abundances greater than the expected errors (the latter judged to be typically 0.05 to $0.08 \mathrm{dex}$ in $[\mathrm{Na}, \mathrm{Mg}, \mathrm{Al}, \mathrm{Si} / \mathrm{Fe}]$ ), in all clusters. Significant variations in $\mathrm{Al}$ were found for all but a few clusters, while variations in $\mathrm{Mg}$ and $\mathrm{Si}$ were found for just a handful of them. The situation was recently 
reviewed by Bastian and Lardo (2017) who demonstrate its complexity in terms of different abundances patterns as well as other relevant cluster properties such as split main-sequences.

In order to investigate further what determines the existence of multiple stellar generations and other factors of possible importance, high precision spectroscopic abundances are important as shown by Yong et al (2013) in a study of 38 red giant stars in NGC 6752. This is one of the globular clusters for which large starto-star abundance variations of $\mathrm{C}, \mathrm{N}, \mathrm{O}, \mathrm{Na}, \mathrm{Mg}$, and $\mathrm{Al}$ have been detected, but which was believed to have no abundance variations for heavier elements. However, in a line-to-line differential analysis of high quality VLT/UVES spectra providing differential abundances with precisions of 0.01 to $0.05 \mathrm{dex}$, Yong et al. found that many of the heavier elements, e.g. Si, Ca, Ti, Y, and Eu, even seem to have abundance variations that are typically a factor of 2 to 3 larger than the measurement errors and positively correlated with the $\mathrm{Na}$ variations. The authors demonstrated that the differential effects found were not a result of differences in effective temperatures of the stars intercompared, nor the result of their choice of reference stars. The resulting abundance variations could be due to variations in the He abundance, because for a fixed mass fraction of heavy elements an increase of the helium mass fraction $(Y)$ causes a corresponding decrease of the hydrogen mass fraction and hence an increase of the abundance of all heavy elements relative to hydrogen. The helium abundance variations in NGC 6752, $\Delta Y=0.03$ as estimated from colour-magnitude diagrams (Milone et al 2013) explain, however, only half of the heavy element abundance variations, so in addition there may be inhomogeneous chemical evolution effects in the proto-cluster gas. Obviously, such high precision studies of other globular clusters would be interesting.

A clear demonstration of abundance scatter in an open cluster beyond the effects caused by stellar evolution discussed above was presented by Liu et al (2016b) for the Hyades cluster; see also this paper for references concerning previous unsucessful attempts in this direction. The authors carried out differential line-by-line analyses of 19 elements in 16 solar-type stars in the cluster, using high-resolution spectra with $\mathrm{S} / \mathrm{N} \sim 350-400$. The intrinsic scatter for an element in between the Hyades stars ( 0.02 to $0.04 \mathrm{dex}$ ) is significantly greater than the average measurement errors (0.01 to $0.02 \mathrm{dex}$ ) for several of the elements, including $\mathrm{Si}, \mathrm{Ca}$, $\mathrm{Fe}$ and $\mathrm{Ni}$, and the different deviations from the mean for all cluster stars for these elements also correlate. The differences show no significant variation with the condensation temperatures of the elements and are found not to be the result of diffusion. Among the possible explanations is an original inhomogeneous composition of the proto-cluster, the explosion and ejection of an early supernova in the cluster-forming cloud, or the pollution by an in-coming gas cloud with different chemical composition.

Another example of abundance differences among members of an open cluster was recently published by Spina et al (2018a). For five solar-type stars in the relatively young (age $\sim 100 \mathrm{Myr}$ ) Pleiades cluster, UVES spectra with a resolving power of $R=75000$ and $S / N \sim 400$ were obtained and used to determine differential abundances of 19 elements with precisions on the order of $0.02 \mathrm{dex}$. The star-to-star abundance differences are clearly correlated with elemental condensation temperature; there are no significant differences for the volatile elements, but for refractory elements with $T_{\mathrm{c}}$ in the range from $1300 \mathrm{~K}$ to $1700 \mathrm{~K}$, the differences (relative to the mean for the Pleiades cluster) range from -0.06 to +0.04 dex. The 
authors suggest that this may be explained by engulfment of rocky planets, i.e. the star with the most negative $T_{\mathrm{c}}$ trend has a stable planetary system (like the Sun), whereas the other stars have accreted different amounts of rocky planetary material leading to an increase of the refractory-to-volatile ratio.

These first results for two of our most near-by open clusters certainly suggest that chemical analyses at similar precision of stars in additional clusters would be interesting.

\section{Abundance-age relations for solar twins}

\subsection{Constraints on nucleosynthesis}

Models of nucleosynthesis and of Galactic chemical evolution are often tested by comparing predicted and observed $[\mathrm{X} / \mathrm{Fe}]-[\mathrm{Fe} / \mathrm{H}]$ relations under the assumption that $[\mathrm{Fe} / \mathrm{H}]$ increases smoothly with time at a given position in the Galaxy. For stars in the solar neighbourhood, there is however, a spread in $[\mathrm{Fe} / \mathrm{H}]$ of more than 0.3 dex at a given age (Edvardsson et al 1993; Nordström et al 2004), which may be due to spurious infall of metal-poor gas or migration of stars in the disk. Therefore, abundance ratios as a function of stellar age provide a more direct test of chemical evolution models. Ages of sufficiently high precision for this purpose may be obtained for solar twins, because the deviation of a star from the Sun in a $\log g-T_{\text {eff }}$ or $L-T_{\text {eff }}$ diagram may be converted to a difference in age via isochrones.

In the following, we will discuss relations between abundance ratios and age for solar twins in the solar neighbourhood obtained from 1D, LTE model atmosphere analyses of HARPS spectra with $R=115000$ and $S / N>500$ by Nissen (2015, 2016) and Nissen et al (2017) for 23 stars and by Spina et al (2018b) and Bedell et al $(2018)$ for 79 stars. The total range in $[\mathrm{Fe} / \mathrm{H}]$ for these samples goes from -0.15 to +0.13 dex, but the large majority of stars have $[\mathrm{Fe} / \mathrm{H}]$ within the \pm 0.10 range used in the definition of a solar twin. Nissen (2015) included nonLTE corrections for some elements, but for solar twins the differential corrections with respect to the Sun are almost negligible. The high precision obtained in these studies is testified by an excellent agreement for 14 stars in common between the two sets of works. The mean differences and standard deviations (Spina \& Bedell Nissen) are: $\Delta T_{\text {eff }}=0 \pm 6 \mathrm{~K}, \Delta \log g=-0.003 \pm 0.014, \Delta[\mathrm{Fe} / \mathrm{H}]=-0.006 \pm 0.005$, and $\Delta$ age $=0.4 \pm 0.5 \mathrm{Gyr}$. For the abundances, the standard deviations of the comparison range from $0.006 \mathrm{dex}$ for the best determined elements ( $\mathrm{Na}, \mathrm{Si}, \mathrm{Ca}$, $\mathrm{Cr}, \mathrm{Ni}$ ) to about 0.010 dex for $\mathrm{C}, \mathrm{Mg}, \mathrm{Al}, \mathrm{S}, \mathrm{Sc}, \mathrm{Ti}, \mathrm{Mn}, \mathrm{Cu}, \mathrm{Zn}, \mathrm{Y}$, and Ba. For oxygen the scatter is higher, i.e. $0.025 \mathrm{dex}$, because of relative large errors of the EW of the weak forbidden O I line at $6300 \AA$ used by Nissen (2015) to derive O abundances. Bedell et al (2018) used instead the O I triplet at $7774 \AA$ in Magellan Telescope MIKE spectra of lower quality than the HARPS spectra applied for the other elements.

9 The age comparison refers to ages derived from Yonsei-Yale isochrones (Demarque et al 2004); as shown by Nissen (2016), ASTEC isochrones (Christensen-Dalsgaard 2008) lead to a somewhat different age scale with $10 \%$ higher ages for the oldest ( 10 Gyr $)$ stars, probably because the ASTEC models include heavy element diffusion in contrast to the Yonsei-Yale models. 

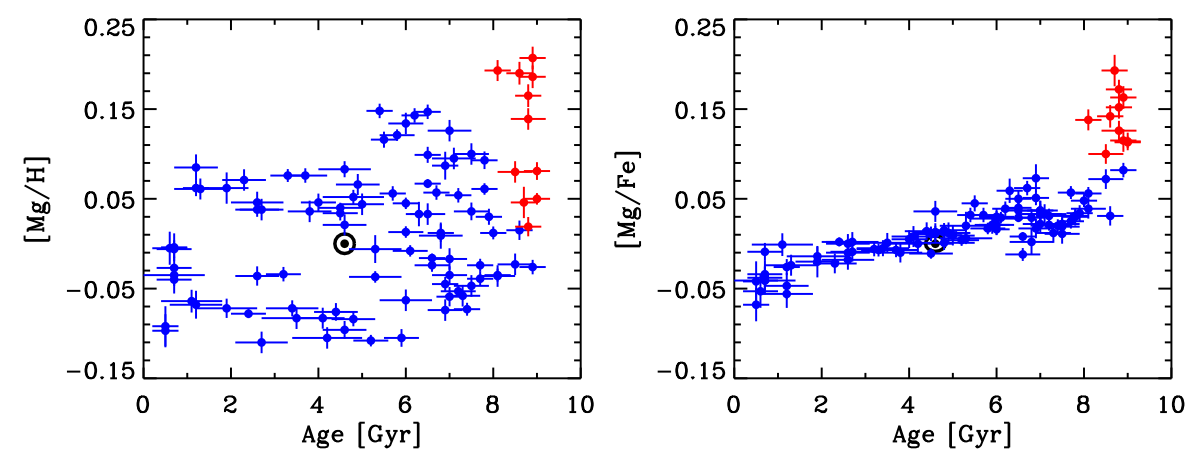

Fig. $13[\mathrm{Mg} / \mathrm{H}]$ and $[\mathrm{Mg} / \mathrm{Fe}]$ of solar twins as a function of stellar age. High-alpha stars $([\mathrm{Mg} / \mathrm{Fe}] \geq 0.1)$ are shown in red color; the others in blue. Based on data from Nissen (2015), Nissen et al (2017), and Bedell et al. (2018).

In the works cited above, age trends of abundance ratios with respect to iron, i.e. $[\mathrm{X} / \mathrm{Fe}]$, have been studied. There are, however, two sources of iron: Type II SNe contribute on a time scale of $\sim 10 \mathrm{Myr}$ while Type Ia SNe on a scale of 1 1.5 Gyr (Matteucci et al 2009). This complicates the interpretation of [X/Fe]-age relations. We will instead consider trends of $[\mathrm{X} / \mathrm{Mg}]$ with age. The main isotope of $\mathrm{Mg},{ }^{24} \mathrm{Mg}$, is made by carbon and neon burning in massive stars and dispersed "instantaneously" to the interstellar medium by Type II SNe. Hence, the $[\mathrm{X} / \mathrm{Mg}]$-age relations may be used to investigate if other elements are also made in high-mass stars or if lower mass stars (including Type Ia $\mathrm{SNe}$ ) make a delayed contribution.

As seen from Fig. 13. $[\mathrm{Mg} / \mathrm{H}]$ of the solar twins has a total spread of about 0.2 dex at all ages. In contrast, there is a remarkable tight relation between $[\mathrm{Mg} / \mathrm{Fe}]$ and age, except for the old 8-9 Gyr stars, among which we have marked high-Mg stars $([\mathrm{Mg} / \mathrm{Fe}]>0.1)$ in red. There is also a hint of a cosmic scatter in $[\mathrm{Mg} / \mathrm{Fe}]$ for the youngest stars and in the age bin 6-7 Gyr, but from 2 to $6 \mathrm{Gyr}$ the rms scatter of $[\mathrm{Mg} / \mathrm{Fe}]$ at a given age is only 0.01 dex, i.e. of the same order of size as the estimated precision of the derived abundances.

The dispersion in the metallicity-age relation for stars in the solar neighbourhood is often explained as due to orbital mixing (migration) of stars born at different galactocentric distances, $R_{G}$, in a Galactic disk with a radial abundance gradient (e.g. Minchev et al 2014). For a gradient of $\delta[\mathrm{Mg} / \mathrm{H}] / \delta R_{G}=-0.05$ dex per kpc, migration should be effective over a Galactic region corresponding to $R_{G}$ from 6 to $10 \mathrm{kpc}$ in order to explain the range in $[\mathrm{Mg} / \mathrm{H}]$ for solar twins in the solar neighbourhood. On the other hand, the Galactic gradient in $[\mathrm{Mg} / \mathrm{Fe}]$ should be vanishingly small in the age range 2 to 6 Gyr to explain the low dispersion in $[\mathrm{Mg} / \mathrm{Fe}]$. This requires that the ratio of Type II and Type Ia SNe has evolved in the same way for $R_{G}$ between 6 and $10 \mathrm{kpc}$. Alternatively, as suggested by Edvardsson et al (1993), the dispersion in $[\mathrm{Mg} / \mathrm{H}]$ could be due to infall of metal-poor gas triggering star formation.

The $[\mathrm{X} / \mathrm{Mg}]$-age relations for some key elements are shown in Figs. 14 and 15. As seen, the scatter at a given age tends to be higher than the estimated errors bars for elements having a condensation temperature significantly lower than that of $\mathrm{Mg}\left(T_{\mathrm{c}}=1336 \mathrm{~K}\right)$, i.e. $\mathrm{C}, \mathrm{O}, \mathrm{Na}, \mathrm{Mn}, \mathrm{Cu}$, and $\mathrm{Zn}$. This may be due to variations in the $[\mathrm{X} / \mathrm{H}]-T_{\mathrm{c}}$ trends related to dust-gas separation in star-forming gas 

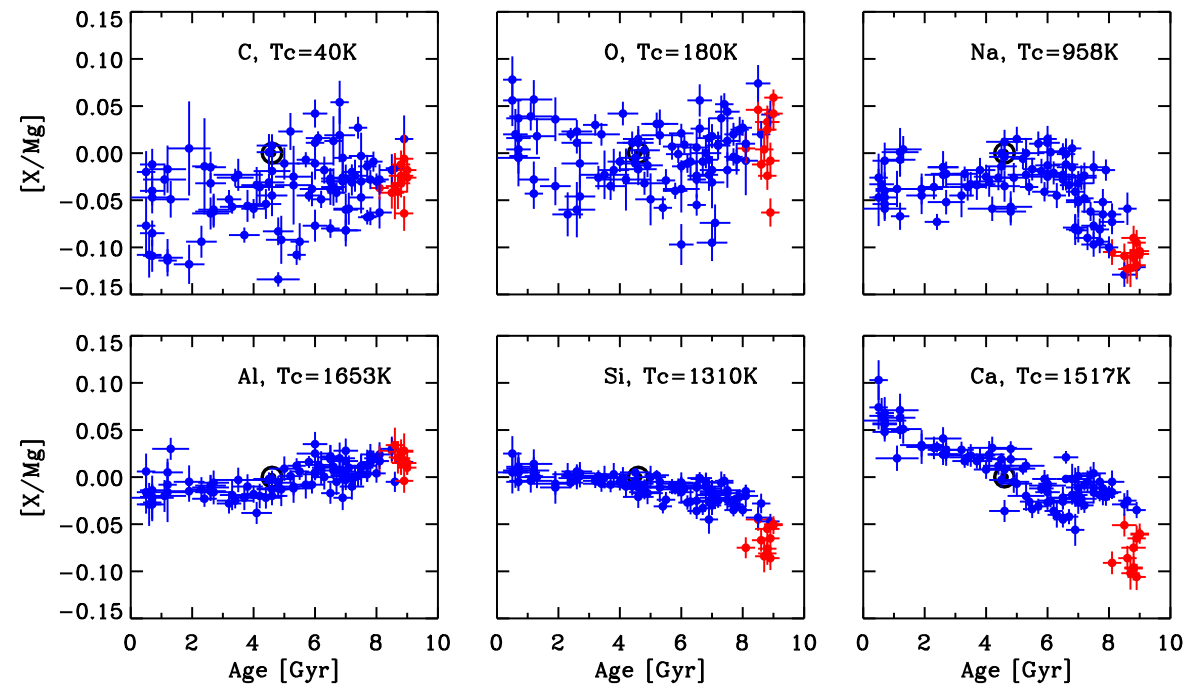

Fig. 14 Abundances of $\mathrm{C}, \mathrm{O}, \mathrm{Na}, \mathrm{Al}, \mathrm{Si}$, and $\mathrm{Ca}$ relative to $\mathrm{Mg}$ as a function of stellar age for solar twins. High-Mg stars are shown in red like in Fig. $13 T_{\mathrm{c}}$ is the elemental condensation temperature in a solar-composition gas (Lodders 2003). Based on data from Nissen (2015), Nissen et al (2017), and Bedell et al. (2018).

clouds. Interestingly, the Sun lies close to the upper envelope of the $[\mathrm{X} / \mathrm{Mg}]$-age distribution for the low- $T_{\mathrm{c}}$ elements, showing (as also discussed in Sect. 4) that the Sun has a high ratio between volatile and refractory elements compared to solar twins with an age around $4.6 \mathrm{Gyr}$.

The high dispersion in $[\mathrm{C} / \mathrm{Mg}]$ and $[\mathrm{O} / \mathrm{Mg}]$ makes it difficult to see if there is an overall trend with age but it does not seem to be the case suggesting that $\mathrm{C}$ and $\mathrm{O}$ are primarily made in massive stars like $\mathrm{Mg}$. In the case of oxygen, this is also predicted from nucleosynthesis calculations, but from chemical evolution modelling of observed $[\mathrm{C} / \mathrm{O}]-[\mathrm{O} / \mathrm{H}]$ and $[\mathrm{C} / \mathrm{Fe}]-[\mathrm{Fe} / \mathrm{H}]$ relations, it has been suggested that low-mass stars have provided up to half of the carbon in the Galactic disk (Chiappini et al 2003; Carigi et al 2005). If so, there should have been a rise of $[\mathrm{C} / \mathrm{Mg}]$ towards younger ages, but if anything, $[\mathrm{C} / \mathrm{Mg}]$ is declining slightly. We conclude that low-mass stars are not contributing significantly to the carbon abundance of solar metallicity disk stars in agreement with the conclusions of Gustafsson et al (1999) based on a study of the [C/O]-[Fe/H] relation for dwarf stars in the solar neighbourhood.

As seen from Fig. 14 $[\mathrm{Na} / \mathrm{Mg}]$ rises steeply with time (i.e. decreasing age) for stars older than about 6 Gyr. Sodium is made by hydrostatic carbon burning in massive stars with a yield that increases with the neutron-excess, which depends on the $\mathrm{C}$ and $\mathrm{O}$ abundances of the Na-producing stars (e.g. Kobayashi et al 2006). Therefore, the rise of $[\mathrm{Na} / \mathrm{Mg}]$ suggests that the chemical evolution in the early Galactic disk was dominated by massive stars with sub-solar abundances of $\mathrm{C}$ and O. For stars younger than about $6 \mathrm{Gyr},[\mathrm{Na} / \mathrm{Mg}]$ is nearly constant indicating that the nucleosynthesis of $\mathrm{Na}$ has mainly occurred in solar-metallicity massive stars. Sodium production in the hydrogen burning shell of low and intermediate mass AGB stars via the Ne-Na cycle (Karakas 2010) does not seem important. 

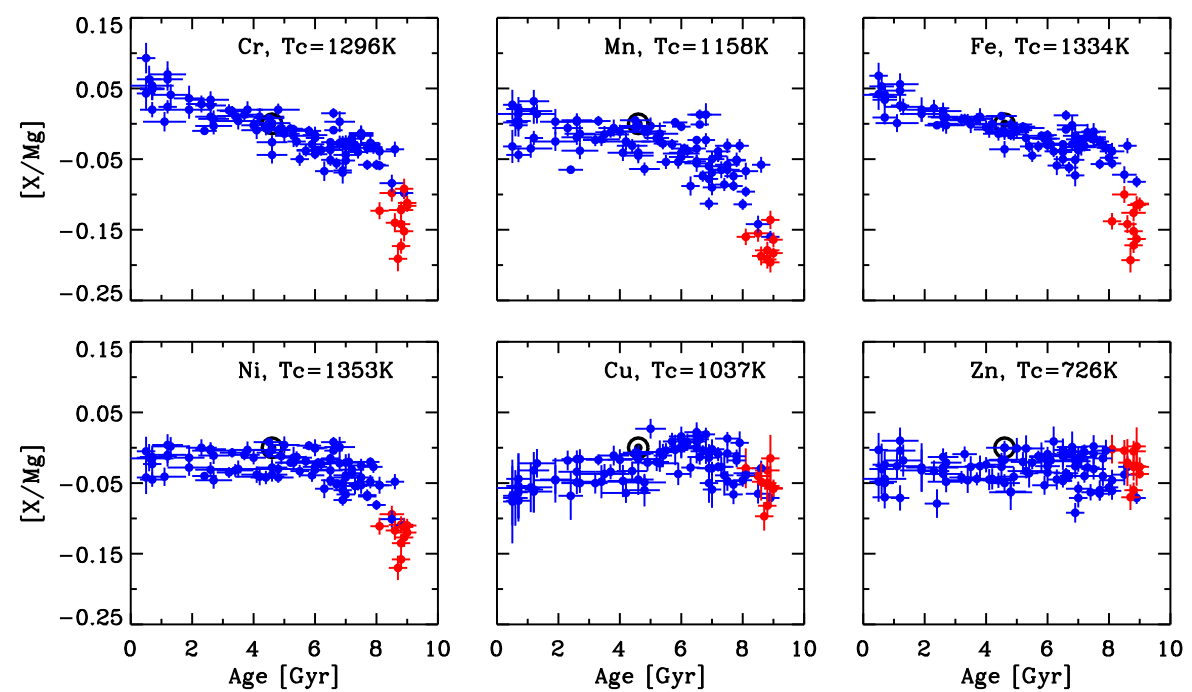

Fig. 15 Abundances of $\mathrm{Cr}, \mathrm{Mn}, \mathrm{Fe}, \mathrm{Ni}, \mathrm{Cu}$, and $\mathrm{Zn}$ relative to $\mathrm{Mg}$ as a function of stellar age for solar twins. Based on data from Nissen (2015, 2016), Nissen et al (2017), and Bedell et al. (2018).

The near-constant trend of $[\mathrm{Al} / \mathrm{Mg}]$ with stellar age supports that aluminium is made in massive stars by carbon and neon burning along with magnesium. $[\mathrm{Si} / \mathrm{Mg}]$ shows a slight rise with time, which is probably due to a non-negligible contribution from Type Ia SNe relative to the contribution from Type II SNe (e.g. Kobavashi et al 2006). Among the other $\alpha$-capture elements, Ti (not shown) behaves like $\mathrm{Si}$, but $\mathrm{Ca}$ is a surprise; as seen from Fig. 14, [Ca/Mg] increases by about 0.15 dex from the oldest to the youngest stars, i.e. similar to the increase of $[\mathrm{Fe} / \mathrm{Mg}]$. Excluding the high-Mg stars (red points), $[\mathrm{Ca} / \mathrm{Fe}]$ is constant as a function of age with a scatter less than 0.01 dex. Thus, the contribution of Type Ia SNe to calcium seems as important as their contribution to iron, which is not predicted by nucleosynthesis calculations (e.g. Kobavashi et al 2006). Alternatively, there could be an additional source of Ca. An intriguing possibility is low-luminosity SNe with presumable low initial masses like SN 2005E for which the yield of $\mathrm{Ca}$ is 5 to 10 times higher than that of classical Type Ia SNe (Perets et al 2010). Frohmaier et al (2018) estimated the volumetric rate of these so-called Carich transients in the local Universe to be about $50 \%$ of the Type Ia SNe rate, which can explain the enhanced $\mathrm{Ca} / \mathrm{Fe}$ ratio in the intracluster medium of galaxy clusters.

The age trends for some of the iron-peak elements are shown in Fig. 15. [Cr/Mg] has nearly the same dependence on age as $[\mathrm{Fe} / \mathrm{Mg}]$ indicating that the ratio of contributions from Type Ia and Type II SNe is about the same for $\mathrm{Cr}$ and Fe. $[\mathrm{Mn} / \mathrm{Mg}]$ and $[\mathrm{Ni} / \mathrm{Mg}]$, on the other hand, show a more flat distribution for ages below 6 Gyr, which is a surprise, because nucleosynthesis calculations predict that the relative contribution of $\mathrm{Mn}$ and $\mathrm{Ni}$ from Type Ia SNe is more important than in the case of $\mathrm{Fe}$ (e.g. Kobavashi et al 2006). [ $\mathrm{Zn} / \mathrm{Mg}]$ is nearly constant over the whole age range, suggesting that $\mathrm{Zn}$ is made in massive stars along with $\mathrm{Mg}$, whereas $[\mathrm{Cu} / \mathrm{Mg}]$ shows a more complicated behaviour, which may reflect 

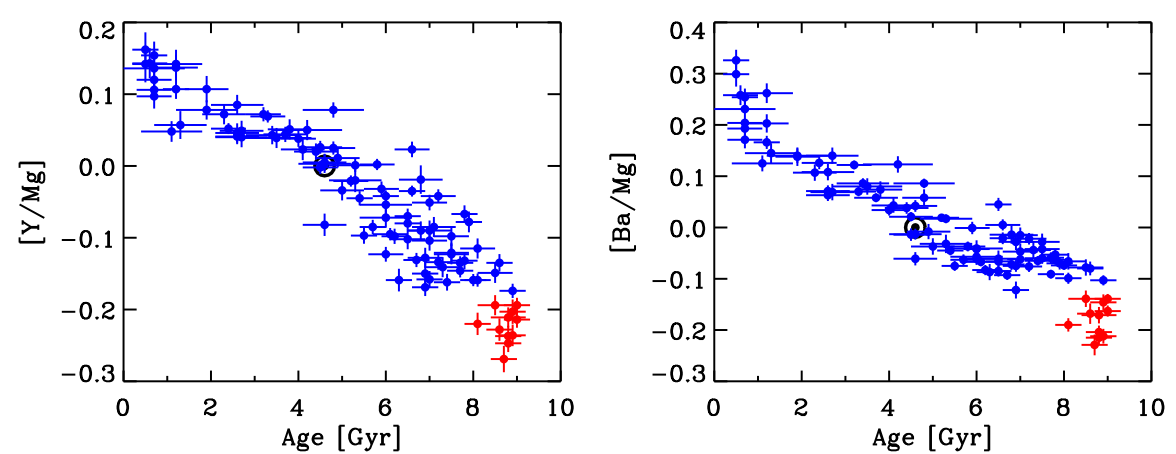

Fig. 16 Abundances of $\mathrm{Y}$ and $\mathrm{Ba}$ relative to $\mathrm{Mg}$ as a function of stellar age for solar twins. Based on data from Nissen (2015, 2016), Nissen et al (2017), and Spina et al (2018b).

that there are several possible ways of $\mathrm{Cu}$ production including the metallicitydependent weak $s$-process in massive stars (Pignatari et al 2010) and explosive nucleosynthesis in Type II SNe (see discussion in Romano and Matteucci 2007).

The age trends of the neutron capture elements $\mathrm{Y}$ and $\mathrm{Ba}$ are shown in Fig. 16. Yttrium represents abundances of elements belonging to the first peak of the $s$-process (the light s-process elements, Sr, Y, and $\mathrm{Zr}$ ) and barium the second peak (the heavy s-process elements, Ba, La, and Ce). As seen both $[\mathrm{Y} / \mathrm{Mg}]$ and $[\mathrm{Ba} / \mathrm{Mg}]$ show a large increase with decreasing age. This rise with time can be ascribed to the importance of the $s$-process in low-mass AGB stars (Busso et al 2001; Maiorca et al 2012; Karakas and Lugaro 2016). As time goes, stars with lower and lower mass contribute to the production of $\mathrm{Y}$ and $\mathrm{Ba}$. There are, however, differences in the evolution of $\mathrm{Y}$ and $\mathrm{Ba}$. $[\mathrm{Y} / \mathrm{Mg}]$ shows the steepest rise with time for the oldest stars, whereas $[\mathrm{Ba} / \mathrm{Mg}]$ is rising more steeply for the youngest stars. As shown by Spina et al (2018b, Fig.8), [Ba/Y] decreases from 9 to 6 Gyr, reaches a minimum around the solar age, and increases with time for the youngest stars. Qualitatively, this behaviour can be explained if the $s$-process elements in the oldest stars come from metal-poor AGB stars with a high ratio between heavy and light s-process elements (Busso et al 2001), whereas the evolution at younger ages is dominated by solar-metallicity AGB stars. According to Karakas and Lugaro (2016, Fig. 15), the heavy-to-light $s$-process ratio increases by $\sim 0.3 \mathrm{dex}$, when the mass of the AGB models decreases from 3 to $1.5 M_{\odot}$.

As seen from Fig. 16. $[\mathrm{Ba} / \mathrm{Mg}]$ has a significant scatter $(\sim 0.2$ dex $)$ among the youngest solar twins (age $<1.5 \mathrm{Gyr}$ ). Furthermore, very large barium abundances, $[\mathrm{Ba} / \mathrm{Fe}] \simeq 0.6$, have been found for $\mathrm{F}$ and $\mathrm{G}$ dwarfs in young open clusters (D'Orazi et al 2009), whereas stars in young associations have lower values, $[\mathrm{Ba} / \mathrm{Fe}] \sim 0.25$, (D'Orazi et al 2012; Reddy and Lambert 2015). According to Reddy and Lambert (2017), this large spread in the derived [Ba/Fe] values for young stars may be spurious due to problems in determining Ba abundances from the rather strong BaII lines available. In a precise study of 24 solar twins with $\mathrm{Ba}$ abundances derived from the Ba II $5853 \AA$ line, they find a correlation between $[\mathrm{Ba} / \mathrm{Fe}]$ and chromospheric activity. They suggest that the derived Ba abundances of the young active stars are too high, because the microturbulence in the upper atmosphere is underestimated in a classical 1D model-atmosphere analysis. Their proposal is supported by the fact that the abundances of other second-peak $s$ - 
process elements, La and Ce, derived from weak lines do not show the same high overabundances as Ba. In general, abundances derived from strong lines formed in the upper part of stellar atmospheres may be systematically in error if analysed with $1 \mathrm{D}$ atmospheres adopting a depth-independent microturbulence parameter. 3D model atmospheres may alleviate this problem, but abundances based on weak lines are to be preferred. Unfortunately, no such lines are available for Ba.

Based on a very tight, linear correlation between $[\mathrm{Y} / \mathrm{Mg}]$ and stellar age for 21 solar twins, Nissen (2015) proposed that the Y/Mg ratio can be used to determine precise ages. According to Fig. 16] based on many more twins, there seems however to be a significant cosmic scatter in the $[\mathrm{Y} / \mathrm{Mg}$ ]-age relation corresponding to $\sim 1$ Gyr in age. Furthermore, Feltzing et al (2017) have shown that [Y/Mg] decreases with $[\mathrm{Fe} / \mathrm{H}]$ at a given age, which makes sense, because at low metallicity there are fewer Fe atoms per neutron to act as seed for the $s$-process. Thus, the use of $[\mathrm{Y} / \mathrm{Mg}]$ or other abundance ratios as chemical clocks would require calibrations including a metallicity term. It should also be emphasized that the relations shown in Fig. 16] refer to stars in the solar neighbourhood and may be different at other Galactocentric distances or in other galaxies with a different chemical evolution history. Thus, Van der Swaelmen et al (2013) find values of [Y/Mg] and $[\mathrm{Ba} / \mathrm{Mg}]$ for solar metallicity stars in the Bar of the Large Magellanic Cloud that are significantly higher that the values for solar twins at any age.

The $[\mathrm{X} / \mathrm{Mg}]$-age relations shown in Figs. 14, 15] and 16 are only valid for solar twin stars, i.e. for a narrow range in metallicity, $-0.1 \lesssim[\mathrm{Fe} / \mathrm{H}] \lesssim 0.1$. At other metallicities, the relations may be different especially for elements with metallicitydependent yields such as $\mathrm{Na}$ and $\mathrm{Y}$. This means that more information on nucleosynthesis and chemical evolution could be obtained if relations between differential abundance ratios and age of the same quality as those for the solar twins were available at other metallicities.

Atomic diffusion of elements is another problem to be considered if abundanceage relations are used as a constraint on chemical evolution models. According to Turcotte et al (1998), the combined effect of gravitational settling and radiative acceleration has decreased the initial abundance of heavy elements in the atmosphere of an $8 \mathrm{Gyr}$ solar twin star by $\sim 12 \%$. The amount differs by up to $2 \%$ between the elements; hence, the effect on the amplitude of $[\mathrm{X} / \mathrm{Mg}]$-age relations shown in Figs. 14 and 15 is less than 0.01 dex. If the stars studied cover a larger range in $T_{\text {eff }}$, differential diffusion effects may be more important. In this connection, we note that diffusion is not affecting the atmospheric composition of $\mathrm{K}$ giants due to their deep convection zones. Therefore, it would be interesting to obtain precise differential abundances of $\mathrm{K}$ giants in clusters with a range of ages in order to test the $[\mathrm{X} / \mathrm{Fe}]-$ age relations obtained for solar twins.

Finally, a few words about lithium. Its abundance, $A(\mathrm{Li})=\log \left(N_{\mathrm{Li}} / N_{\mathrm{H}}\right)+12$, in the atmospheres of FGK dwarfs and subgiants depends on stellar mass and age due to proton-capture destruction of $\mathrm{Li}$ at the bottom of the convection zone and on stellar age because of Galactic evolution of Li (see e.g. Prantzos et al 2017). For solar twins with masses close to $1 M_{\odot}$, precise lithium abundances have been derived from the the Li I $6708 \AA$ resonance line in high S/N HARPS spectra, but conclusions from different works do not agree. Delgado Mena et al (2014) find no significant correlation of $A(\mathrm{Li})$ with age for stars older than $\sim 2$ Gyr but a lower average Li abundance of planet hosting stars than the Li abundance of stars without detected planets, which they ascribe to additional rotationally induced 
mixing at the bottom of the convection zone in stars with planets. Carlos et al $(\underline{2016})$, on the other hand, find a declining trend of Li abundance with age for the majority of solar twins (including the Sun and a few stars with detected planets), i.e. from $A(\mathrm{Li}) \simeq 2.2$ at $1 \mathrm{Gyr}$ to $A(\mathrm{Li}) \simeq 0.6$ dex at $8 \mathrm{Gyr}$. The trend corresponds reasonable well to predictions of $\mathrm{Li}$ depletion in $1 M_{\odot}$ stellar models with nonstandard mixing calibrated on the Sun.

\subsection{Constraints on exoplanet compositions}

The relations between abundance ratios and age for solar twins may also be used as constraints on the composition of planets around stars. In addition to mass and radius, the proportions of $\mathrm{C}, \mathrm{O}$, and rock-forming elements like $\mathrm{Mg}, \mathrm{Si}$, and $\mathrm{Fe}$ determines the structure, mineralogy, and geodynamics of terrestrial planets and therefore should be taken into account when discussing the potential for habitability (e.g. Bond et al 2010; Carter-Bond et al 2012; Unterborn et al 2014; Dorn et al 2015; Thiabaud et al 2015). Of particular importance are the $\mathrm{C} / \mathrm{O}$ number ratio. The Sun has $\mathrm{C} / \mathrm{O}=0.55 \pm 0.08$ (Asplund et al 2009), but if a host star and hence the proto-planetary disk has $\mathrm{C} / \mathrm{O} \gtrsim 0.8$ there is a possibility for the existence of "Carbon" planets containing large amounts of graphite and carbides instead of Earth-like silicates (Kuchner and Seager 2005; Bond et al 2010). The Mg/Si ratio is also important; the Sun has $\mathrm{Mg} / \mathrm{Si}=1.20 \pm 0.14$ (Scott et al 2015), but if the protoplanetary disk has $\mathrm{Mg} / \mathrm{Si}$ less than 1.0 , terrestrial planets will have a Mg-depleted mineralogy different from that of the Earth (Carter-Bond et al 2012).

Determinations of C/O ratios by Delgado Mena et al (2010) and Petigura and Marcy (2011) suggested that a substantial fraction of solar-type stars have $\mathrm{C} / \mathrm{O}>0.8$, but later more precise work did not confirm this (Nissen 2013; Nissen et al 2014; Brewer and Fischer 2016; Suárez-Andrés et al 2018); C/O rises with $[\mathrm{Fe} / \mathrm{H}]$ but stays below 0.8 even at the highest metallicities, $[\mathrm{Fe} / \mathrm{H}] \sim+0.4$. For solar twins, $\mathrm{C} / \mathrm{O}$ is also well below 0.8 as seen from Fig. 17 There is some indication of a cosmic scatter in $\mathrm{C} / \mathrm{O}$ at a given age and a decline of $\mathrm{C} / \mathrm{O}$ towards young ages. This trend agrees with the low $\mathrm{C} / \mathrm{O}$ ratio $(\mathrm{C} / \mathrm{O}=0.37 \pm 0.05)$ for young B-type stars in the solar neighbourhood determined by Nieva and Przybilla (2012) from a careful non-LTE study of high-S/N spectra. They interpreted the low $\mathrm{C} / \mathrm{O}$ of $\mathrm{B}$ stars relative to the solar ratio in terms of a strong radial gradient of $\mathrm{C} / \mathrm{O}$ in the Galactic disk combined with migration of the Sun from an assumed birthplace at a distance of $5-6 \mathrm{kpc}$ from the Galactic centre. The data for solar twins suggest, however, that the difference in $\mathrm{C} / \mathrm{O}$ may instead be due to the difference in age between the Sun and young B stars.

As the $\mathrm{Mg}$ and $\mathrm{Si}$ abundances in solar-type stars are close to each other, it requires high-precision data to determine if $\mathrm{Mg} / \mathrm{Si}$ in a given star is above or below 1.0. In a 1D, LTE abundance study of $849 \mathrm{~F}, \mathrm{G}$, and $\mathrm{K}$ dwarfs in the solar neighbourhood based on high-S/N KECK/HIRES spectra, Brewer and Fischer (2016) found a $\mathrm{Mg} / \mathrm{Si}$ distribution significantly broader than the estimated error distribution and concluded that about $40 \%$ of the stars have $\mathrm{Mg} / \mathrm{Si}<1.0$. Suárez-Andrés et al (2018) also found $\mathrm{Mg} / \mathrm{Si}<1.0$ values in an analysis of 499 solar-type stars with high-S/N HARPS spectra; $15 \%$ of the stars harbouring highmass planets have $\mathrm{Mg} / \mathrm{Si}<1.0$ and $\mathrm{Mg} / \mathrm{Si}$ decreases with increasing $[\mathrm{Fe} / \mathrm{H}]$ reaching $\sim 1.0$ at $[\mathrm{Fe} / \mathrm{H}]=+0.3$. On the other hand, it is seen from Fig. 17 that the 

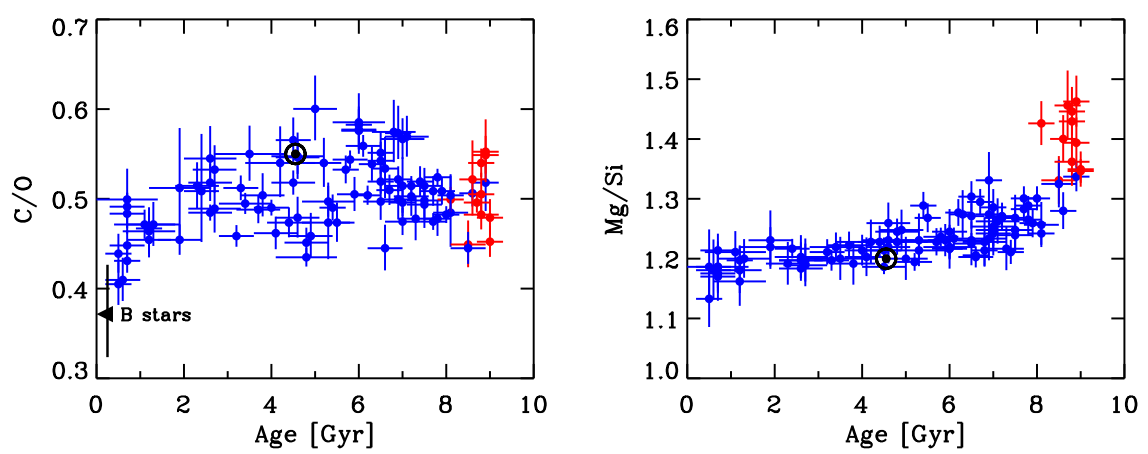

Fig. 17 The $\mathrm{C} / \mathrm{O}$ and $\mathrm{Mg} / \mathrm{Si}$ number ratios in solar twins as a function of stellar age. Error bars refer to values relative to the solar ratios $\mathrm{C} / \mathrm{O}=0.55 \pm 0.08$ (Asplund et al 2009) and $\mathrm{Mg} / \mathrm{Si}=1.20 \pm 0.14$ (Scott et al 2015). In the left panel, the $\mathrm{C} / \mathrm{O}$ ratio in young B-type stars in the solar neighbourhood (Nieva and Przybilla 2012) is marked. Based on data from Nissen (2015), Nissen et al (2017), and Bedell et al (2018).

$\mathrm{Mg} / \mathrm{Si}$ ratio in solar twins is well above 1.0 when adopting a reference solar ratio of $\mathrm{Mg} / \mathrm{Si}=1.20 \pm 0.14$ as derived by Scott et al (2015) from an analysis of photospheric lines based on 1D non-LTE corrections to 3D LTE synthetic spectra. If instead the meteoritic ratio of $\mathrm{CI}$ carbonaceous chondrites, $\mathrm{Mg} / \mathrm{Si}=1.05 \pm 0.05$ (Lodders 2003), is adopted for the solar reference value as in Brewer and Fischer (2016) and Suárez-Andrés et al (2018), the Mg/Si ratio of solar twins approaches 1.0 for the youngest stars but only one of these stars has $\mathrm{Mg} / \mathrm{Si}<1.0$.

We conclude that the solar twin data provide no evidence for the existence of stars with $\mathrm{C} / \mathrm{O}>0.8$ or $\mathrm{Mg} / \mathrm{Si}<1.0$, which suggests that terrestrial planets around solar metallicity stars have compositions similar to that of the Earth. The more metal-rich stars, those with $[\mathrm{Fe} / \mathrm{H}] \sim+0.3$, are more likely to have terrestrial planets with a composition and structure different from that of the Earth, but detailed 3D, non-LTE analyses are needed to improve the accuracy of the derived abundances for the corresponding stars. Further 3D, non-LTE studies of the $\mathrm{Mg} / \mathrm{Si}$ in the Sun would also be interesting given that the photospheric ratio is higher than the ratio in meteorites.

Considering that several terrestrial planets have been discovered in orbits around $\mathrm{M}$ dwarfs, e.g. one planet around Proxima Centauri (Anglada-Escudé et al 2016) and seven planets around TRAPPIST-1 (Gillon et al 2017), and that many more are expected to be discovered during the TESS and Plato missions, there is a high interest in determining precise parameters of $M$ dwarfs. In particular, it would be interesting to determine ages of $M$ dwarfs from abundance ratios that are closely correlated with age due to Galactic chemical evolution (see Sect. 6.1). As discussed in Sect. 2.7 $[\alpha / \mathrm{Fe}]$ may be determined with internal precisions of $\sim 0.05$ dex from near-infrared spectra of $\mathrm{M}$ dwarfs. It remains, however, to be seen if this precision can also be obtained for elemental ratios that are sensitive to age, such as $s$-process elements relative to $\alpha$-capture elements, e.g. Y/Mg. 


\section{Conclusions and recommendations}

The quantitative chemical analysis of stars has developed in a very impressive way. With pioneering work in the 1920'ies and 1930'es by Pavne (1928), Russell (1929), Unsöld (1938), Strömgren (1940), and others, it provided some important basic arguments for the famous $\mathrm{B}^{2} \mathrm{FH}$ article (Burbidge et al 1957) on the synthesis of elements in stars. Yet, in the 1960'ies the results of the analyses were often found to be in error by more than a factor of two, due to systematic errors which were difficult to judge. This made it even possible, as late as in the early 1970'ies (Worrall 1972), to question whether the abundances should at all be taken seriously. The rather low quality of abundance data was realized by many of the spectroscopists active then; even if they in some cases found rather high internal precision in their studies, the possible systematic errors often raised question marks, even among optimists.

Since then, however, the situation has improved, and, as in other areas of science, this is to a great extent due to improvements in technology. Larger telescopes, more efficient spectrometers, detectors with linear response, new algorithms and computers for ever better and more detailed modeling and data handling, and great improvements in atomic and molecular data, in all these respects the situation is now totally changed. It is also important to realize the fundamental contribution by a number of individuals, often specialists with limited recognition outside their own speciality areas, who made the progress possible by devoted work on spectral line identifications, transition rates, non-LTE radiative transfer, turbulent convection, spectrometer stability, detector development, data-base handling, etc.

Due to this development, and collaborative work, the differential relative errors in certain chemical abundance measures are now sometimes only a few percent, or even less, as has been illustrated in this review. It is important to note then that these numbers refer to differential errors, i.e. abundance differences relative to a similar star, like the Sun for solar twins or analogues, or a component in a binary which is almost identical to its companion. The reason for this limitation of the present paper is that hardly any absolute astrophysical abundance is known to the prescribed accuracy of better than 0.04 dex. Even for the Sun, the absolute errors, in spite of excellent observed spectra, 3D models and detailed non-LTE calculations that can be checked versus detailed observations of the solar disk, we have still to expect somewhat larger absolute errors for almost all elements. This is also underlined by an external check that is available, the comparison with the estimate of the sound speed in the solar interior Basu and Antia 2013; Vinyoles et al 2017), which still deviates significantly from what one would expect from the photospheric abundances of C and O Asplund et al 2009; Steffen et al 2015; Amarsi et al 2018a).

It is also important to realize that accurate absolute abundances are needed in many astrophysical applications. In studies of the action of the s-process in the interior of AGB stars and the transportation of these elements to the surface of the stars, the true amounts of these elements matter for, e.g. a proper understanding of their production in the Galaxy. Similarly, the yields of carbon from AGB stars of various types, from Wolf-Rayet stars and from supernovae, and the accurate carbon abundances in stars of different ages and populations in the Galaxy, are needed for a proper understanding of the carbon production as a whole. The same holds true for many chemical elements, and not the least regarding their produc- 
tion in the early Galaxy, studies where absolute abundances have to be measured for stars very different from the Sun. Although relative abundances of for instance Pop II stars still may reveal interesting structures and give insight into the degree of mixing and frequency of supernovae of different types, a quantitative understanding of such processes will require absolute abundances. Also, the study of other processes beyond nucleosynthesis will benefit from absolute abundances of high quality, and at least free from severe systematic errors. Among those processes is "diffusion" - or sooner the interaction of radiative pressure, turbulent and other mixing, and sinking in the gravitational field - in the surface layers of stars. Also, the consequences of planetary interaction, a subject that has been developed efficiently by differential studies as seen above, will need absolute abundances when applied to a wider range of spectral types and to single stars. Of particular interest here are the $\mathrm{M}$ dwarfs which have so different spectra and atmospheric structures that truly differential analyses cannot be extended very far in temperature.

It is certainly easy to ask for more measurements in higher accuracy, but is it really worth the effort? A sweeping argument here would be to refer to William Thomson, 1st Baron Kelvin, who in a famous quote on the need to measure argued that what you cannot express in numbers "may be the beginning of knowledge, but you have scarcely ... advanced to the stage of science" (Thomson 1889). A more precise argument is that the evolution of the abundance determination for stars have proven to continuously generate new and unexpected knowledge when the accuracy has been raised. This is illustrated above in numerous cases. So, we have no reason to believe that this development would stop when we take further steps in the development towards high-precision abundances.

Thus, we conclude that the area of absolute abundance determination should be further developed. This will require full 3D modelling of stellar atmospheres, and ultimately also with statistical-equilibrium in the models themselves and not only the spectra, and further on with magneto-hydrodynamic effects accounted for properly. One important step towards such models will also be a detailed and critical comparison with high-resolution high $\mathrm{S} / \mathrm{N}$ spectra extending from UV to far IR for a number of different standard stars.

In the continuing efforts to improve the accuracy and advance the abundance analysis to new types of stars, the work by atomic and molecular physicists in improving the necessary basic data will be of fundamental significance. Such efforts must be enthusiastically encouraged and adequately supported by the astrophysics community.

A very impressive development has taken place during recent decades not only in the accuracy of the analyses but also in the number of stars analysed. In the 1960 's, a PhD thesis could contain the analysis of one single star. Present samples may contain several hundreds, and in some cases even thousands of stars, and several tens of elements to be analysed for each of them. Some of these large projects unfortunately suffer from inhomogeneities, in instrumentation, reduction methods of the observations, or analysis; for a discussion, see for example Jofré et al $(2017 \mathrm{~b})$. According to our experience, it is indeed very important for the usefulness of a study to keep the methodology constant. Another important directive when large surveys are to be planned is certainly to use strict and well defined selection criteria. In order to avoid inhomogeneities in methods and samples, merging of different data sets should be avoided (which certainly does not mean that one should not inter-compare the sets critically). 
A promising but as yet rather primitive approach towards the very extensive and rapidly expanding data sets of stellar spectra, is to develop automatic analysis systems. It is our impression that considerable efforts should be made to advance such algorithms before they can be left alone, freed from the critical watching eyes of experienced astronomers. In particular it must be important to design them to report on stars and stellar groups that depart from the typical stars in their training samples. The great homogeneous surveys of present-day astronomy open up the possibilities for making discoveries of new totally unexpected phenomena in nature. We should not miss that opportunity.

In planning extensive surveys, it is important to carefully balance the need for a large sample of stars against the spectral resolution and the S/N necessary for reaching the accuracy aimed for. It is not enough to trade a decrease in accuracy in the results for individual stars against the formal accuracy in the mean for these stars. Such means may be plagued by considerable systematic errors, e.g. in the continuum definition or due to blends if the $\mathrm{S} / \mathrm{N}$ or the resolution are not high enough, see Lindegren and Feltzing (2013) for an illustrative discussion of this. In the choice between great samples of stars and relatively resolved spectra at high $\mathrm{S} / \mathrm{N}$ of a smaller number of stars, the latter is often to be preferred and can in many cases be realized with small losses of representativity if care is exercised in the definition of the sample.

Acknowledgements The anonymous referee as well as Anish Amarsi, Paula Jofré, Karin Lind, and Jorge Meléndez are thanked for critical reading of a first version of the manuscript and for many helpful suggestions for improvements. Funding for the Stellar Astrophysics Centre is provided by the Danish National Research Foundation (Grant DNRF106).

\section{References}

Abbott BP, Abbott R, Abbott TD, Acernese F, Ackley K, Adams C, Adams T, Addesso P, Adhikari RX, Adya VB, et al (2017) GW170817: Observation of Gravitational Waves from a Binary Neutron Star Inspiral. Physical Review Letters 119(16):161101, DOI 10. 1103/PhysRevLett.119.161101, arXiv:1710.05832

Adams FC (2010) The Birth Environment of the Solar System. ARA\&A 48:47-85, DOI 10. 1146/annurev-astro-081309-130830, arXiv:1001.5444

Adibekyan VZ, Sousa SG, Santos NC, Delgado Mena E, González Hernández JI, Israelian G, Mayor M, Khachatryan G (2012) Chemical abundances of 1111 FGK stars from the HARPS GTO planet search program. Galactic stellar populations and planets. A\&A 545:A32, DOI 10.1051/0004-6361/201219401, arXiv:1207.2388

Adibekyan VZ, González Hernández JI, Delgado Mena E, Sousa SG, Santos NC, Israelian G, Figueira P, Bertran de Lis S (2014) On the origin of stars with and without planets. $\mathrm{T}_{c}$ trends and clues to Galactic evolution. A\&A 564:L15, DOI 10.1051/0004-6361/201423435, arXiv:1404.4514

Allard F, Homeier D, Freytag B (2012) Models of very-low-mass stars, brown dwarfs and exoplanets. Philosophical Transactions of the Royal Society of London Series A 370:27652777, DOI 10.1098/rsta.2011.0269, arXiv:1112.3591

Amarsi AM, Asplund M (2017) The solar silicon abundance based on 3D non-LTE calculations. MNRAS 464:264-273, DOI 10.1093/mnras/stw2445, arXiv:1609.07283

Amarsi AM, Asplund M, Collet R, Leenaarts J (2016a) Non-LTE oxygen line formation in 3D hydrodynamic model stellar atmospheres. MNRAS 455:3735-3751, DOI 10.1093/mnras/ stv2608, arXiv:1511.01155

Amarsi AM, Lind K, Asplund M, Barklem PS, Collet R (2016b) Non-LTE line formation of Fe in late-type stars - III. 3D non-LTE analysis of metal-poor stars. MNRAS 463:1518-1533, DOI 10.1093/mnras/stw2077, arXiv:1608.06390 
Amarsi AM, Barklem PS, Asplund M, Collet R, Zatsarinny O (2018a) Inelastic O+H collisions and the O I $777 \mathrm{~nm}$ solar centre-to-limb variation. A\&A 616:A89, DOI 10.1051/0004-6361/ 201832770, arXiv:1803.10531

Amarsi AM, Nordlander T, Barklem PS, Asplund M, Collet R, Lind K (2018b) Effective temperature determinations of late-type stars based on 3D non-LTE Balmer line formation. A\&A 615:A139, DOI 10.1051/0004-6361/201732546, arXiv:1804.02305

Anglada-Escudé G, Amado PJ, Barnes J, Berdiñas ZM, Butler RP, Coleman GAL, de La Cueva I, Dreizler S, Endl M, Giesers Bea (2016) A terrestrial planet candidate in a temperate orbit around Proxima Centauri. Nature 536:437-440, DOI 10.1038/nature19106, arXiv: 1609.03449

Anstee SD, O'Mara BJ (1991) An investigation of Brueckner's theory of line broadening with application to the sodium D lines. MNRAS 253:549-560, DOI 10.1093/mnras/253.3.549

Anstee SD, O'Mara BJ (1995) Width cross-sections for collisional broadening of s-p and p-s transitions by atomic hydrogen. MNRAS 276:859-866, DOI 10.1093/mnras/276.3.859

Argast D, Samland M, Gerhard OE, Thielemann FK (2000) Metal-poor halo stars as tracers of ISM mixing processes during halo formation. A\&A 356:873-887, astro-ph/9911178

Arnone E, Ryan SG, Argast D, Norris JE, Beers TC (2005) Mg abundances in metal-poor halo stars as a tracer of early Galactic mixing. A\&A 430:507-522, DOI 10.1051/0004-6361: 20041034, astro-ph/0410180

Asplund M (2005) New Light on Stellar Abundance Analyses: Departures from LTE and Homogeneity. ARA\&A 43:481-530, DOI 10.1146/annurev.astro.42.053102.134001

Asplund M, Ludwig HG, Nordlund A, Stein RF (2000a) The effects of numerical resolution on hydrodynamical surface convection simulations and spectral line formation. A\&A 359:669681 , astro-ph/0005319

Asplund M, Nordlund A, Trampedach R, Allende Prieto C, Stein RF (2000b) Line formation in solar granulation. I. Fe line shapes, shifts and asymmetries. A\&A 359:729-742, astro-ph/0005320

Asplund M, Grevesse N, Sauval AJ, Scott P (2009) The Chemical Composition of the Sun. ARA\&A 47:481-522, DOI 10.1146/annurev.astro.46.060407.145222, arXiv:0909.0948

Baraffe I, Chabrier G (2010) Effect of episodic accretion on the structure and the lithium depletion of low-mass stars and planet-hosting stars. A\&A 521:A44, DOI 10.1051/0004-6361/ 201014979, arXiv: 1008.4288

Barklem PS (2016a) Accurate abundance analysis of late-type stars: advances in atomic physics. A\&A Rev 24:9, DOI 10.1007/s00159-016-0095-9, arXiv: 1604.07659

Barklem PS (2016b) Excitation and charge transfer in low-energy hydrogen-atom collisions with neutral atoms: Theory, comparisons, and application to Ca. Phys. Rev. A 93(4):042705, DOI 10.1103/PhysRevA.93.042705, arXiv:1603.07097

Barklem PS (2018) Excitation and charge transfer in low-energy hydrogen atom collisions with neutral iron. A\&A 612:A90, DOI 10.1051/0004-6361/201732365, arXiv:1801.07050

Barklem PS, Piskunov N, O'Mara BJ (2000) Self-broadening in Balmer line wing formation in stellar atmospheres. A\&A 363:1091-1105, astro-ph/0010022

Baschek B (1959) Aufbau und chemische Zusammensetzung der Atmosphäre des Subdwarfs HD 140283. Mit 10 Textabbildungen. Zeitschrift für Astrophysik 48:95

Bastian N, Lardo C (2017) Multiple Stellar Populations in Globular Clusters. ArXiv e-prints arXiv: 1712.01286

Basu S, Antia HM (2004) Constraining Solar Abundances Using Helioseismology. ApJL 606:L85-L88, DOI 10.1086/421110, astro-ph/0403485

Basu S, Antia HM (2013) Revisiting the Issue of Solar Abundances. In: Journal of Physics Conference Series, Journal of Physics Conference Series, vol 440, p 012017, DOI 10.1088/ 1742-6596/440/1/012017

Beck PG, Allende Prieto C, Van Reeth T, Tkachenko A, Raskin G, van Winckel H, do Nascimento JD Jr, Salabert D, Corsaro E, García RA (2016) The HERMES solar atlas and the spectroscopic analysis of the seismic solar analogue KIC 3241581. A\&A 589:A27, DOI 10.1051/0004-6361/201425423, arXiv:1511.06583

Bedell M, Meléndez J, Bean JL, Ramírez I, Leite P, Asplund M (2014) Stellar Chemical Abundances: In Pursuit of the Highest Achievable Precision. ApJ 795:23, DOI 10.1088/ 0004-637X/795/1/23, arXiv:1409.1230

Bedell M, Bean JL, Meléndez J, Spina L, Ramírez I, Asplund M, Alves-Brito A, dos Santos L, Dreizler S, Yong D, Monroe T, Casagrande L (2018) The Chemical Homogeneity of Sun-like Stars in the Solar Neighborhood. ApJ 865:68, DOI 10.3847/1538-4357/aad908, 
arXiv: 1802.02576

Bell RA (1970) The calibration of narrow band photometry - I. Cambridge observations of G and K giants. MNRAS 148:25-52, DOI 10.1093/mnras/148.1.25

Bell RA, Dickens RJ (1980) Chemical abundances in the globular clusters M3, M13, and NGC 6752. ApJ 242:657-672, DOI 10.1086/158502

Bell RA, Edvardsson B, Gustafsson B (1985) The surface gravity of Arcturus from MgH lines, strong metal lines and the ionization equilibrium of iron. MNRAS 212:497-515, DOI $10.1093 / \mathrm{mnras} / 212.3 .497$

Bensby T, Feltzing S, Lundström I, Ilyin I (2005) $\alpha_{-}^{-}, \mathrm{r}-$, and s-process element trends in the Galactic thin and thick disks. A\&A 433:185-203, DOI 10.1051/0004-6361:20040332, astro-ph/0412132

Bensby T, Feltzing S, Oey MS (2014) Exploring the Milky Way stellar disk. A detailed elemental abundance study of $714 \mathrm{~F}$ and $\mathrm{G}$ dwarf stars in the solar neighbourhood. A\&A 562:A71, DOI 10.1051/0004-6361/201322631, arXiv:1309.2631

Bensby T, Feltzing S, Gould A, Yee JC, Johnson JA, Asplund M, Meléndez J, Lucatello S, Howes LM, McWilliam A, et al (2017) Chemical evolution of the Galactic bulge as traced by microlensed dwarf and subgiant stars. VI. Age and abundance structure of the stellar populations in the central sub-kpc of the Milky Way. A\&A 605:A89, DOI 10.1051/0004-6361/201730560, arXiv:1702.02971

Bergemann M (2011) Ionization balance of $\mathrm{Ti}$ in the photospheres of the Sun and four late-type stars. MNRAS 413:2184-2198, DOI 10.1111/j.1365-2966.2011.18295.x, arXiv:1101.0828

Bergemann M, Lind K, Collet R, Magic Z, Asplund M (2012) Non-LTE line formation of Fe in late-type stars - I. Standard stars with $1 \mathrm{D}$ and $\langle 3 \mathrm{D}\rangle$ model atmospheres. MNRAS 427:2749, DOI 10.1111/j.1365-2966.2012.21687.x, arXiv:1207.2455

Bergemann M, Kudritzki RP, Würl M, Plez B, Davies B, Gazak Z (2013) Red Supergiant Stars as Cosmic Abundance Probes. II. NLTE Effects in J-band Silicon Lines. ApJ 764:115, DOI 10.1088/0004-637X/764/2/115, arXiv:1212.2649

Bergemann M, Collet R, Amarsi AM, Kovalev M, Ruchti G, Magic Z (2017) Non-local Thermodynamic Equilibrium Stellar Spectroscopy with 1D and $\langle 3 \mathrm{D}\rangle$ Models. I. Methods and Application to Magnesium Abundances in Standard Stars. ApJ 847:15, DOI 10.3847/1538-4357/aa88cb, arXiv:1612.07355

Bertelli Motta C, Pasquali A, Richer J, Michaud G, Salaris M, Bragaglia A, Magrini L, Randich S, Grebel EK, Adibekyan V, et al (2018) The Gaia-ESO Survey: evidence of atomic diffusion in M67? MNRAS 478:425-438, DOI 10.1093/mnras/sty1011, arXiv:1804.06293

Bertran de Lis S, Allende Prieto C, Majewski SR, Schiavon RP, Holtzman JA, Shetrone M, Carrera R, García Pérez AE, Mészáros S, Frinchaboy PM, et al (2016) Cosmic variance in $[\mathrm{O} / \mathrm{Fe}]$ in the Galactic disk. A\&A 590:A74, DOI 10.1051/0004-6361/201527827, arXiv:1603.05491

Biazzo K, Gratton R, Desidera S, Lucatello S, Sozzetti A, Bonomo AS, Damasso M, Gandolfi D, Affer L, Boccato C, et al (2015) The GAPS programme with HARPS-N at TNG. X. Differential abundances in the XO-2 planet-hosting binary. A\&A 583:A135, DOI 10.1051/ 0004-6361/201526375, arXiv: 1506.01614

Biermann L (1932) Untersuchungen über den inneren Aufbau der Sterne. IV. Konvektionszonen im Innern der Sterne. (Veröffentlichungen der Universitäts-Sternwarte Göttingen, Nr. 27. ) Mit 5 Abbildungen. Zeitschrift für Astrophysik 5:117

Blackwell DE, Willis RB (1977) Stellar gravities from metallic line profiles, with application to Arcturus - The effective temperature of Arcturus. MNRAS 180:169-176, DOI 10.1093/ mnras/180.2.169

Blanco-Cuaresma S, Soubiran C, Heiter U, Jofré P (2014) Determining stellar atmospheric parameters and chemical abundances of FGK stars with iSpec. A\&A 569:A111, DOI 10.1051/0004-6361/201423945, arXiv:1407.2608

Blanco-Cuaresma S, Soubiran C, Heiter U, Asplund M, Carraro G, Costado MT, Feltzing S, González-Hernández JI, Jiménez-Esteban F, Korn AJ, et al (2015) Testing the chemical tagging technique with open clusters. A\&A 577:A47, DOI 10.1051/0004-6361/201425232, arXiv: 1503.02082

Bland-Hawthorn J, Sharma S (2016) GALAH Survey: Chemical tagging and disk reconstruction. Astronomische Nachrichten 337:894, DOI 10.1002/asna.201612393

Bland-Hawthorn J, Karlsson T, Sharma S, Krumholz M, Silk J (2010) The Chemical Signatures of the First Star Clusters in the Universe. ApJ 721:582-596, DOI 10.1088/0004-637X/721/ 1/582, arXiv: 1007.5374 
Boesgaard AM, Tripicco MJ (1986) Lithium in the Hyades Cluster. ApJL 302:L49-L53, DOI $10.1086 / 184635$

Bond JC, O'Brien DP, Lauretta DS (2010) The Compositional Diversity of Extrasolar Terrestrial Planets. I. In Situ Simulations. ApJ 715:1050-1070, DOI 10.1088/0004-637X/715/2/ 1050, arXiv: 1004.0971

Brewer JM, Fischer DA (2016) C/O and Mg/Si Ratios of Stars in the Solar Neighborhood. ApJ 831:20, DOI 10.3847/0004-637X/831/1/20

Brewer JM, Fischer DA, Basu S, Valenti JA, Piskunov N (2015) Accurate Gravities of F, G, and K stars from High Resolution Spectra Without External Constraints. ApJ 805:126, DOI 10.1088/0004-637X/805/2/126, arXiv:1503.07180

Brewer JM, Fischer DA, Valenti JA, Piskunov N (2016) Spectral Properties of Cool Stars: Extended Abundance Analysis of 1,617 Planet-search Stars. ApJS 225:32, DOI 10.3847/ 0067-0049/225/2/32, arXiv:1606.07929

Buder S, Asplund M, Duong L, Kos J, Lind K, Ness MK, Sharma S, Bland-Hawthorn J, Casey AR, De Silva GM, et al (2018) The GALAH Survey: second data release. MNRAS 478:4513-4552, DOI 10.1093/mnras/sty1281, arXiv:1804.06041

Burbidge EM, Burbidge GR, Fowler WA, Hoyle F (1957) Synthesis of the Elements in Stars. Reviews of Modern Physics 29:547-650, DOI 10.1103/RevModPhys.29.547

Busso M, Gallino R, Lambert DL, Travaglio C, Smith VV (2001) Nucleosynthesis and Mixing on the Asymptotic Giant Branch. III. Predicted and Observed s-Process Abundances. ApJ 557:802-821, DOI 10.1086/322258, astro-ph/0104424

Carigi L, Peimbert M, Esteban C, García-Rojas J (2005) Carbon, Nitrogen, and Oxygen Galactic Gradients: A Solution to the Carbon Enrichment Problem. ApJ 623:213-224, DOI 10.1086/428491, astro-ph/0408398

Carlos M, Nissen PE, Meléndez J (2016) Correlation between lithium abundances and ages of solar twin stars. A\&A 587:A100, DOI 10.1051/0004-6361/201527478, arXiv:1601.05054

Carretta E, Bragaglia A, Gratton R, Lucatello S (2009) Na-O anticorrelation and HB. VIII. Proton-capture elements and metallicities in 17 globular clusters from UVES spectra. A\&A 505:139-155, DOI 10.1051/0004-6361/200912097, arXiv:0909.2941

Carter-Bond JC, O'Brien DP, Delgado Mena E, Israelian G, Santos NC, González Hernández JI (2012) Low Mg/Si Planetary Host Stars and Their Mg-depleted Terrestrial Planets. ApJL 747:L2, DOI 10.1088/2041-8205/747/1/L2, arXiv:1201.1939

Casagrande L, Ramírez I, Meléndez J, Bessell M, Asplund M (2010) An absolutely calibrated $T_{\text {eff }}$ scale from the infrared flux method. Dwarfs and subgiants. A\&A 512:A54, DOI 10.1051/0004-6361/200913204, arXiv:1001.3142

Castelli F, Kurucz RL (2003) New Grids of ATLAS9 Model Atmospheres. In: Piskunov N, Weiss WW, Gray DF (eds) Modelling of Stellar Atmospheres, IAU Symposium, vol 210, p A20

Cayrel R (1969) Comparison of Synthetic Spectra with Real Spectra. In: Gingerich O (ed) Theory and Observation of Normal Stellar Atmospheres, p 237

Cayrel R, Depagne E, Spite M, Hill V, Spite F, François P, Plez B, Beers T, Primas F, Andersen $\mathrm{J}$, et al (2004) First stars V - Abundance patterns from C to Zn and supernova yields in the early Galaxy. A\&A 416:1117-1138, DOI 10.1051/0004-6361:20034074, astro-ph/0311082

Cayrel R, van't Veer-Menneret C, Allard NF, Stehlé C (2011) The H $\alpha$ Balmer line as an effective temperature criterion. I. Calibration using $1 \mathrm{D}$ model stellar atmospheres. A\&A 531:A83, DOI 10.1051/0004-6361/201116911

Cayrel de Strobel G (1996) Stars resembling the Sun. A\&A Rev 7:243-288, DOI 10.1007/ s001590050006

Cayrel de Strobel G, Knowles N, Hernandez G, Bentolila C (1981) In search of real solar twins. A\&A 94:1-11

Cescutti G, Romano D, Matteucci F, Chiappini C, Hirschi R (2015) The role of neutron star mergers in the chemical evolution of the Galactic halo. A\&A 577:A139, DOI 10.1051/ 0004-6361/201525698, arXiv:1503.02954

Chamberlain JW, Aller LH (1951) The Atmospheres of A-Type Subdwarfs and 95 Leonis. ApJ 114:52, DOI 10.1086/145451

Chiappini C, Romano D, Matteucci F (2003) Oxygen, carbon and nitrogen evolution in galaxies. MNRAS 339:63-81, DOI 10.1046/j.1365-8711.2003.06154.x, astro-ph/0209627

Chiavassa A, Casagrande L, Collet R, Magic Z, Bigot L, Thévenin F, Asplund M (2018) The STAGGER-grid: A grid of 3D stellar atmosphere models. V. Synthetic stellar spectra and broad-band photometry. A\&A 611:A11, DOI 10.1051/0004-6361/201732147, 
arXiv: 1801.01895

Christensen-Dalsgaard J (2008) ASTEC, the Aarhus STellar Evolution Code. Ap\&SS 316:1324, DOI 10.1007/s10509-007-9675-5, arXiv:0710.3114

Christensen-Dalsgaard J, Proffitt CR, Thompson MJ (1993) Effects of diffusion on solar models and their oscillation frequencies. ApJL 403:L75-L78, DOI 10.1086/186725

Cochran WD, Hatzes AP, Butler RP, Marcy GW (1997) The Discovery of a Planetary Companion to 16 Cygni B. ApJ 483:457-463, astro-ph/9611230

Cohen JG (1978) Abundances in globular cluster red giants. I - M3 and M13. ApJ 223:487-508, DOI $10.1086 / 156284$

Collet R, Magic Z, Asplund M (2011) The StaggerGrid project: a grid of 3-D model atmospheres for high-precision spectroscopy. In: Journal of Physics Conference Series, Journal of Physics Conference Series, vol 328, p 012003, DOI 10.1088/1742-6596/328/1/012003, arXiv: 1110.5475

Côté B, Fryer CL, Belczynski K, Korobkin O, Chruślińska M, Vassh N, Mumpower MR, Lippuner J, Sprouse TM, Surman R, Wollaeger R (2018) The Origin of r-process Elements in the Milky Way. ApJ 855:99, DOI 10.3847/1538-4357/aaad67, arXiv:1710.05875

Delgado Mena E, Israelian G, González Hernández JI, Bond JC, Santos NC, Udry S, Mayor M (2010) Chemical Clues on the Formation of Planetary Systems: C/O Versus $\mathrm{Mg} / \mathrm{Si}$ for HARPS GTO Sample. ApJ 725:2349-2358, DOI 10.1088/0004-637X/725/2/2349, arXiv: 1009.5224

Delgado Mena E, Israelian G, González Hernández JI, Sousa SG, Mortier A, Santos NC, Adibekyan VZ, Fernandes J, Rebolo R, Udry S, Mayor M (2014) Li depletion in solar analogues with exoplanets. Extending the sample. A\&A 562:A92, DOI 10.1051/0004-6361/ 201321493, arXiv:1311.6414

Demarque P, Woo JH, Kim YC, Yi SK (2004) Y² Isochrones with an Improved Core Overshoot Treatment. ApJS 155:667-674, DOI 10.1086/424966

Do T, Kerzendorf W, Konopacky Q, Marcinik JM, Ghez A, Lu JR, Morris MR (2018) Supersolar Metallicity Stars in the Galactic Center Nuclear Star Cluster: Unusual Sc, V, and Y Abundances. ApJL 855:L5, DOI 10.3847/2041-8213/aaaec3, arXiv:1802.08270

D’Orazi V, Magrini L, Randich S, Galli D, Busso M, Sestito P (2009) Enhanced Production of Barium in Low-Mass Stars: Evidence from Open Clusters. ApJL 693:L31-L34, DOI 10.1088/0004-637X/693/1/L31, arXiv:0901.2743

D’Orazi V, Biazzo K, Desidera S, Covino E, Andrievsky SM, Gratton RG (2012) The chemical composition of nearby young associations: s-process element abundances in AB Doradus, Carina-Near and Ursa Major. MNRAS 423:2789-2799, DOI 10.1111/j.1365-2966. 2012.21088.x, arXiv:1204.2635

Dorn C, Khan A, Heng K, Connolly JAD, Alibert Y, Benz W, Tackley P (2015) Can we constrain the interior structure of rocky exoplanets from mass and radius measurements? A\&A 577:A83, DOI 10.1051/0004-6361/201424915, arXiv:1502.03605

Dotter A, Conroy C, Cargile P, Asplund M (2017) The Influence of Atomic Diffusion on Stellar Ages and Chemical Tagging. ApJ 840:99, DOI 10.3847/1538-4357/aa6d10, arXiv: 1704.03465

Dravins D, Nordlund A (1990) Stellar Granulation - Part Five - Synthetic Spectral Lines in Disk Integrated Starlight. A\&A 228:203

Edvardsson B, Andersen J, Gustafsson B, Lambert DL, Nissen PE, Tomkin J (1993) The Chemical Evolution of the Galactic Disk - Part One - Analysis and Results. A\&A 275:101

Eggen OJ, Lynden-Bell D, Sandage AR (1962) Evidence from the motions of old stars that the Galaxy collapsed. ApJ 136:748, DOI 10.1086/147433

Epstein CR, Johnson JA, Dong S, Udalski A, Gould A, Becker G (2010) Chemical Composition of Faint (I $21 \mathrm{mag}$ ) Microlensed Bulge Dwarf OGLE-2007-BLG-514S. ApJ 709:447-457, DOI 10.1088/0004-637X/709/1/447, arXiv:0910.1358

Fabbian D, Moreno-Insertis F (2015) Continuum Intensity and [O I] Spectral Line Profiles in Solar 3D Photospheric Models: The Effect of Magnetic Fields. ApJ 802:96, DOI 10.1088/ 0004-637X/802/2/96, arXiv:1501.06916

Fabbian D, Moreno-Insertis F, Khomenko E, Nordlund A (2012) Solar Fe abundance and magnetic fields. Towards a consistent reference metallicity. A\&A 548:A35, DOI 10.1051/ 0004-6361/201219335, arXiv:1209.2771

Feltzing S, Howes LM, McMillan PJ, Stonkute E (2017) On the metallicity dependence of the [Y/Mg]-age relation for solar-type stars. MNRAS 465:L109-L113, DOI 10.1093/mnrasl/ slw209, arXiv: 1610.03852 
Fernández-Alvar E, Carigi L, Schuster WJ, Hayes CR, Ávila-Vergara N, Majewski SR, Allende Prieto C, Beers TC, Sánchez SF, Zamora O, et al (2018) Disentangling the Galactic Halo with APOGEE. II. Chemical and Star Formation Histories for the Two Distinct Populations. ApJ 852:50, DOI 10.3847/1538-4357/aa9ced, arXiv:1711.06225

Fischel D (1964) On the Influence of the Chemical Composition on the Structure and Metal Index of Stellar Atmospheres. ApJ 140:221, DOI 10.1086/147909

Flores M, González JF, Jaque Arancibia M, Buccino A, Saffe C (2016) Discovery of an activity cycle in the solar analog HD 45184. Exploring Balmer and metallic lines as activity proxy candidates. A\&A 589:A135, DOI 10.1051/0004-6361/201628145, arXiv:1604.01307

François P, Depagne E, Hill V, Spite M, Spite F, Plez B, Beers TC, Andersen J, James G, Barbuy B, et al (2007) First stars. VIII. Enrichment of the neutron-capture elements in the early Galaxy. A\&A 476:935-950, DOI 10.1051/0004-6361:20077706, arXiv:0709.3454

Frebel A, Norris JE (2015) Near-Field Cosmology with Extremely Metal-Poor Stars. ARA\&A 53:631-688, DOI 10.1146/annurev-astro-082214-122423, arXiv:1501.06921

Freeman K, Bland-Hawthorn J (2002) The New Galaxy: Signatures of Its Formation. ARA\&A 40:487-537, DOI 10.1146/annurev.astro.40.060401.093840, astro-ph/0208106

Freytag B, Steffen M, Ludwig HG, Wedemeyer-Böhm S, Schaffenberger W, Steiner O (2012) Simulations of stellar convection with CO5BOLD. Journal of Computational Physics 231:919-959, DOI 10.1016/j.jcp.2011.09.026, arXiv:1110.6844

Frohmaier C, Sullivan M, Maguire K, Nugent P (2018) The Volumetric Rate of Calciumrich Transients in the Local Universe. ApJ 858:50, DOI 10.3847/1538-4357/aabc0b, arXiv: 1804.03103

Fuhrmann K (1998) Nearby stars of the Galactic disk and halo. A\&A 338:161-183

Fuhrmann K (2011) Nearby stars of the Galactic disc and halo - V. MNRAS 414:2893-2922, DOI 10.1111/j.1365-2966.2011.18476.x

Fuhrmann K, Chini R, Kaderhandt L, Chen Z (2017) On the local stellar populations. MNRAS 464:2610-2621, DOI 10.1093/mnras/stw2526

Fulbright JP (2002) Abundances and Kinematics of Field Stars. II. Kinematics and Abundance Relationships. AJ 123:404-412, DOI 10.1086/324630, astro-ph/0110164

Gaia Collaboration, Brown AGA, Vallenari A, Prusti T, de Bruijne JHJ, Babusiaux C, BailerJones CAL, Biermann M, Evans DW, Eyer L, et al (2018) Gaia Data Release 2. Summary of the contents and survey properties. A\&A 616:A1, DOI 10.1051/0004-6361/201833051, arXiv: 1804.09365

García Pérez AE, Allende Prieto C, Holtzman JA, Shetrone M, Mészáros S, Bizyaev D, Carrera R, Cunha K, García-Hernández DA, Johnson JA, et al (2016) ASPCAP: The APOGEE Stellar Parameter and Chemical Abundances Pipeline. AJ 151:144, DOI 10.3847/0004-6256/151/6/144, arXiv:1510.07635

Genovali K, Lemasle B, da Silva R, Bono G, Fabrizio M, Bergemann M, Buonanno R, Ferraro I, François P, Iannicola G, et al (2015) On the $\alpha$-element gradients of the Galactic thin disk using Cepheids. A\&A 580:A17, DOI 10.1051/0004-6361/201525894, arXiv:1503.03758

Gillon M, Triaud AHMJ, Demory BO, Jehin E, Agol E, Deck KM, Lederer SM, de Wit J, Burdanov A, Ingalls JGea (2017) Seven temperate terrestrial planets around the nearby ultracool dwarf star TRAPPIST-1. Nature 542:456-460, DOI 10.1038/nature21360, arXiv: 1703.01424

Gilmore G, Reid N (1983) New light on faint stars. III - Galactic structure towards the South Pole and the Galactic thick disc. MNRAS 202:1025-1047, DOI 10.1093/mnras/202.4.1025

Gilmore G, Wyse RFG (1998) Element Ratios and the Formation of the Stellar Halo. AJ 116:748-753, DOI 10.1086/300459, astro-ph/9805144

Gilmore G, Randich S, Asplund M, Binney J, Bonifacio P, Drew J, Feltzing S, Ferguson A, Jeffries R, Micela G, et al (2012) The Gaia-ESO Public Spectroscopic Survey. The Messenger 147:25-31

Gonzalez G (1998) Spectroscopic analyses of the parent stars of extrasolar planetary system candidates. A\&A 334:221-238

Gonzalez G, Laws C, Tyagi S, Reddy BE (2001) Parent Stars of Extrasolar Planets. VI. Abundance Analyses of 20 New Systems. AJ 121:432-452, DOI 10.1086/318048, astro-ph/0010197

Gonzalez G, Carlson MK, Tobin RW (2010) Parent stars of extrasolar planets - XI. Trends with condensation temperature revisited. MNRAS 407:314-320, DOI 10.1111/j.1365-2966. 2010.16900.x, arXiv:1004.3313

Gratton RG, Carretta E, Matteucci F, Sneden C (2000) Abundances of light elements in metal- 
poor stars. IV. $[\mathrm{Fe} / \mathrm{O}]$ and $[\mathrm{Fe} / \mathrm{Mg}]$ ratios and the history of star formation in the solar neighborhood. A\&A 358:671-681, astro-ph/0004157

Gratton RG, Bonanno G, Claudi RU, Cosentino R, Desidera S, Lucatello S, Scuderi S (2001a) Non-interacting main-sequence binaries with different chemical compositions: Evidences of infall of rocky material? A\&A 377:123-131, DOI 10.1051/0004-6361:20011066

Gratton RG, Bonifacio P, Bragaglia A, Carretta E, Castellani V, Centurion M, Chieffi A, Claudi R, Clementini G, D'Antona F, et al (2001b) The O-Na and Mg-Al anticorrelations in turn-off and early subgiants in globular clusters. A\&A 369:87-98, DOI 10.1051/0004-6361: 20010144, astro-ph/0012457

Grevesse N, Asplund M, Sauval AJ (2007) The Solar Chemical Composition. SSRv 130:105114, DOI $10.1007 / \mathrm{s} 11214-007-9173-7$

Gruyters P, Nordlander T, Korn AJ (2014) Atomic diffusion and mixing in old stars. V. A deeper look into the globular cluster NGC 6752. A\&A 567:A72, DOI 10.1051/0004-6361/ 201423590, arXiv: 1405.6543

Gustafsson B (1989) Chemical analyses of cool stars. ARA\&A 27:701-756, DOI 10.1146/ annurev.aa.27.090189.003413

Gustafsson B (2018a) Dust cleansing of star-forming gas. I. Has radiation from bright stars affected the chemical composition of the Sun and M 67? A\&A 616:A91, DOI 10.1051/ 0004-6361/201732354, arXiv:1805.00547

Gustafsson B (2018b) Dust cleansing of star-forming gas: II. Did late accretion flows change the chemical composition of the solar atmosphere? ArXiv e-prints arXiv:1809.02361

Gustafsson B, Bell RA, Eriksson K, Nordlund A (1975) A grid of model atmospheres for metal-deficient giant stars. I. A\&A 42:407-432

Gustafsson B, Karlsson T, Olsson E, Edvardsson B, Ryde N (1999) The origin of carbon, investigated by spectral analysis of solar-type stars in the Galactic Disk. A\&A 342:426439, astro-ph/9811303

Gustafsson B, Edvardsson B, Eriksson K, Jørgensen UG, Nordlund Å, Plez B (2008) A grid of MARCS model atmospheres for late-type stars. I. Methods and general properties. A\&A 486:951-970, DOI 10.1051/0004-6361:200809724, arXiv:0805.0554

Hauschildt PH, Allard F, Baron E (1999a) The NextGen Model Atmosphere Grid for $3000<T_{\text {eff }}\langle 10,000 \mathrm{~K}$. ApJ 512:377-385, DOI 10.1086/306745, astro-ph/9807286

Hauschildt PH, Allard F, Ferguson J, Baron E, Alexander DR (1999b) The NEXTGEN Model Atmosphere Grid. II. Spherically Symmetric Model Atmospheres for Giant Stars with Effective Temperatures between 3000 and 6800 K. ApJ 525:871-880, DOI 10.1086/307954, astro-ph/9907194

Hawkins K, Jofré P, Masseron T, Gilmore G (2015) Using chemical tagging to redefine the interface of the Galactic disc and halo. MNRAS 453:758-774, DOI 10.1093/mnras/stv1586, arXiv: 1507.03604

Hayden MR, Bovy J, Holtzman JA, Nidever DL, Bird JC, Weinberg DH, Andrews BH, Majewski SR, Allende Prieto C, Anders F, et al (2015) Chemical Cartography with APOGEE: Metallicity Distribution Functions and the Chemical Structure of the Milky Way Disk. ApJ 808:132, DOI 10.1088/0004-637X/808/2/132, arXiv:1503.02110

Hayes CR, Majewski SR, Shetrone M, Fernández-Alvar E, Allende Prieto C, Schuster WJ, Carigi L, Cunha K, Smith VV, Sobeck J, et al (2018) Disentangling the Galactic Halo with APOGEE. I. Chemical and Kinematical Investigation of Distinct Metal-poor Populations. ApJ 852:49, DOI 10.3847/1538-4357/aa9cec, arXiv:1711.05781

Haywood M, Di Matteo P, Lehnert MD, Katz D, Gómez A (2013) The age structure of stellar populations in the solar vicinity. Clues of a two-phase formation history of the Milky Way disk. A\&A 560:A109, DOI 10.1051/0004-6361/201321397, arXiv:1305.4663

Haywood M, Di Matteo P, Lehnert MD, Snaith O, Khoperskov S, Gómez A (2018) In Disguise or Out of Reach: First Clues about In Situ and Accreted Stars in the Stellar Halo of the Milky Way from Gaia DR2. ApJ 863:113, DOI 10.3847/1538-4357/aad235, arXiv: 1805.02617

Helfer HL, Wallerstein G, Greenstein JL (1959) Abundances in Some Population II K Giants. ApJ 129:700, DOI 10.1086/146668

Helmi A, Babusiaux C, Koppelman HH, Massari D, Veljanoski J, Brown AGA (2018) The formation of two of the major structural components of the Milky Way. ArXiv e-prints arXiv: 1806.06038

Henyey L, Vardya MS, Bodenheimer P (1965) Studies in Stellar Evolution. III. The Calculation of Model Envelopes. ApJ 142:841, DOI 10.1086/148357 
Hinkle K, Wallace L, Livingston W (1995) Infrared Atlas of the Arcturus Spectrum, 0.9-5.3 microns. PASP 107:1042, DOI 10.1086/133660

Ho AYQ, Ness MK, Hogg DW, Rix HW, Liu C, Yang F, Zhang Y, Hou Y, Wang Y (2017a) Label Transfer from APOGEE to LAMOST: Precise Stellar Parameters for 450,000 LAMOST Giants. ApJ 836:5, DOI 10.3847/1538-4357/836/1/5, arXiv:1602.00303

Ho AYQ, Rix HW, Ness MK, Hogg DW, Liu C, Ting YS (2017b) Masses and Ages for 230,000 LAMOST Giants, via Their Carbon and Nitrogen Abundances. ApJ 841:40, DOI 10.3847/ 1538-4357/aa6db3, arXiv:1609.03195

Hogg DW, Casey AR, Ness M, Rix HW, Foreman-Mackey D, Hasselquist S, Ho AYQ, Holtzman JA, Majewski SR, Martell SL, et al (2016) Chemical Tagging Can Work: Identification of Stellar Phase-space Structures Purely by Chemical-abundance Similarity. ApJ 833:262, DOI 10.3847/1538-4357/833/2/262, arXiv:1601.05413

Holtzman JA, Shetrone M, Johnson JA, Allende Prieto C, Anders F, Andrews B, Beers TC, Bizyaev D, Blanton MR, Bovy J, et al (2015) Abundances, Stellar Parameters, and Spectra from the SDSS-III/APOGEE Survey. AJ 150:148, DOI 10.1088/0004-6256/150/5/148, arXiv: 1501.04110

Ishigaki M, Chiba M, Aoki W (2010) Chemical Abundances of Outer Halo Stars in the Milky Way. PASJ 62:143-178, DOI 10.1093/pasj/62.1.143, arXiv:0912.0329

Jofré P, Das P, Bertranpetit J, Foley R (2017a) Cosmic phylogeny: reconstructing the chemical history of the solar neighbourhood with an evolutionary tree. MNRAS 467:1140-1153, DOI 10.1093/mnras/stx075, arXiv:1611.02575

Jofré P, Heiter U, Buder S (2017b) Gaia FGK benchmark stars: a bridge between spectroscopic surveys. In: Astronomical Society of India Conference Series, Astronomical Society of India Conference Series, vol 14, pp 37-44, arXiv:1709.09366

Kallinger T, Hekker S, Garcia RA, Huber D, Matthews JM (2016) Precise stellar surface gravities from the time scales of convectively driven brightness variations. Science Advances $2: 1500654$, DOI 10.1126/sciadv.1500654

Karakas AI (2010) Updated stellar yields from asymptotic giant branch models. MNRAS 403:1413-1425, DOI 10.1111/j.1365-2966.2009.16198.x, arXiv:0912.2142

Karakas AI, Lugaro M (2016) Stellar Yields from Metal-rich Asymptotic Giant Branch Models. ApJ 825:26, DOI 10.3847/0004-637X/825/1/26, arXiv:1604.02178

Karlsson T, Gustafsson B (2005) Stochastic chemical enrichment in metal-poor systems. II. Abundance ratios and scatter. A\&A 436:879-894, DOI 10.1051/0004-6361:20042168, astro-ph/0504617

Kirby EN, Guhathakurta P, Bolte M, Sneden C, Geha MC (2009) Multi-element Abundance Measurements from Medium-resolution Spectra. I. The Sculptor Dwarf Spheroidal Galaxy. ApJ 705:328-346, DOI 10.1088/0004-637X/705/1/328, arXiv:0909.3092

Kiselman D, Pereira TMD, Gustafsson B, Asplund M, Meléndez J, Langhans K (2011) Is the solar spectrum latitude-dependent?. An investigation with SST/TRIPPEL. A\&A 535:A14, DOI 10.1051/0004-6361/201117553, arXiv:1108.4527

Kobayashi C, Umeda H, Nomoto K, Tominaga N, Ohkubo T (2006) Galactic Chemical Evolution: Carbon through Zinc. ApJ 653:1145-1171, DOI 10.1086/508914, astro-ph/0608688

Koppelman H, Helmi A, Veljanoski J (2018) One Large Blob and Many Streams Frosting the nearby Stellar Halo in Gaia DR2. ApJL 860:L11, DOI 10.3847/2041-8213/aac882, arXiv: 1804.11347

Korn AJ, Grundahl F, Richard O, Mashonkina L, Barklem PS, Collet R, Gustafsson B, Piskunov N (2007) Atomic Diffusion and Mixing in Old Stars. I. Very Large Telescope FLAMES-UVES Observations of Stars in NGC 6397. ApJ 671:402-419, DOI 10.1086/523098, arXiv:0709.0639

Korotin SA, Andrievsky SM, Zhukova AV (2018) Copper abundance from Cu I and Cu II lines in metal-poor star spectra: NLTE versus LTE. MNRAS 480:965-971, DOI 10.1093/ mnras/sty1886, arXiv: 1807.08972

Kraft RP (1994) Abundance differences among globular-cluster giants: Primordial versus evolutionary scenarios. PASP 106:553-565, DOI 10.1086/133416

Kraft RP, Sneden C, Langer GE, Shetrone MD (1993) Oxygen abundaces in Halo giants. IV The oxygen-sodium anticorrelation in a sample of 22 bright giants in M13. AJ 106:14901507, DOI 10.1086/116742

Krumholz MR, Matzner CD (2009) The Dynamics of Radiation-pressure-dominated H II Regions. ApJ 703:1352-1362, DOI 10.1088/0004-637X/703/2/1352, arXiv:0906.4343

Kuchner MJ, Seager S (2005) Extrasolar Carbon Planets. ArXiv Astrophysics e-prints 
astro-ph/0504214

Kunitomo M, Guillot T, Ida S, Takeuchi T (2018) Revisiting the pre-main-sequence evolution of stars II. Consequences of planet formation on stellar surface composition. ArXiv e-prints arXiv: 1808.07396

Kurucz RL (1979) Model atmospheres for G, F, A, B, and O stars. ApJS 40:1-340, DOI $10.1086 / 190589$

Kurucz RL (2005) New atlases for solar flux, irradiance, central intensity, and limb intensity. Memorie della Societa Astronomica Italiana Supplementi 8:189

Lambert DL, Brown JA, Hinkle KH, Johnson HR (1984) Carbon, nitrogen, and oxygen abundances in Betelgeuse. ApJ 284:223-237, DOI 10.1086/162401

Lambert DL, Gustafsson B, Eriksson K, Hinkle KH (1986) The chemical composition of carbon stars. I - Carbon, nitrogen, and oxygen in 30 cool carbon stars in the Galactic disk. ApJS 62:373-425, DOI 10.1086/191145

Laws C, Gonzalez G (2001) A Differential Spectroscopic Analysis of 16 Cygni A and B. ApJ 553:405-409, DOI 10.1086/320669, astro-ph/0101304

Letarte B, Hill V, Tolstoy E, Jablonka P, Shetrone M, Venn KA, Spite M, Irwin MJ, Battaglia G, Helmi A, et al (2010) A high-resolution VLT/FLAMES study of individual stars in the centre of the Fornax dwarf spheroidal galaxy. A\&A 523:A17, DOI 10.1051/0004-6361/ 200913413, arXiv: 1007.1007

Lind K, Charbonnel C, Decressin T, Primas F, Grundahl F, Asplund M (2011) Tracing the evolution of NGC 6397 through the chemical composition of its stellar populations. A\&A 527:A148, DOI 10.1051/0004-6361/201015356, arXiv:1012.0477

Lind K, Bergemann M, Asplund M (2012) Non-LTE line formation of Fe in late-type stars - II. 1D spectroscopic stellar parameters. MNRAS 427:50-60, DOI 10.1111/j.1365-2966.2012. 21686.x, arXiv: 1207.2454

Lind K, Amarsi AM, Asplund M, Barklem PS, Bautista M, Bergemann M, Collet R, Kiselman D, Leenaarts J, Pereira TMD (2017) Non-LTE line formation of Fe in late-type stars IV. Modelling of the solar centre-to-limb variation in 3D. MNRAS 468:4311-4322, DOI 10.1093/mnras/stx673, arXiv:1703.04027

Lindegren L, Feltzing S (2013) The case for high precision in elemental abundances of stars in the era of large spectroscopic surveys. A\&A 553:A94, DOI 10.1051/0004-6361/201321057, arXiv: 1304.3409

Lindgren S, Heiter U (2017) Metallicity determination of M dwarfs. Expanded parameter range in metallicity and effective temperature. A\&A 604:A97, DOI 10.1051/0004-6361/ 201730715 , arXiv: 1705.08785

Lindgren S, Heiter U, Seifahrt A (2016) Metallicity determination of M dwarfs. Highresolution infrared spectroscopy. A\&A 586:A100, DOI 10.1051/0004-6361/201526602, arXiv:1510.06642

Liu F, Asplund M, Yong D, Meléndez J, Ramírez I, Karakas AI, Carlos M, Marino AF (2016a) The chemical compositions of solar twins in the open cluster M67. MNRAS 463:696-704, DOI 10.1093/mnras/stw2045, arXiv:1608.03788

Liu F, Yong D, Asplund M, Ramírez I, Meléndez J (2016b) The Hyades open cluster is chemically inhomogeneous. MNRAS 457:3934-3948, DOI 10.1093/mnras/stw247, arXiv: 1601.07354

Liu F, Yong D, Asplund M, Feltzing S, Mustill AJ, Meléndez J, Ramírez I, Lin J (2018) Detailed chemical compositions of the wide binary HD 80606/80607: revised stellar properties and constraints on planet formation. A\&A 614:A138, DOI 10.1051/0004-6361/201832701, arXiv: 1802.09306

Livingston W, Wallace L (1991) An atlas of the solar spectrum in the infrared from 1850 to 9000 cm-1 (1.1 to 5.4 micrometer). NSO Technical Report, Tucson: National Solar Observatory, National Optical Astronomy Observatory

Lodders K (2003) Solar System Abundances and Condensation Temperatures of the Elements. ApJ 591:1220-1247, DOI 10.1086/375492

Ludwig HG, Behara NT, Steffen M, Bonifacio P (2009a) Impact of granulation effects on the use of Balmer lines as temperature indicators. A\&A 502:L1-L4, DOI 10.1051/0004-6361/ 200911997, arXiv:0906.4697

Ludwig HG, Caffau E, Steffen M, Freytag B, Bonifacio P, Kučinskas A (2009b) The CIFIST 3D model atmosphere grid. Mem. Soc. Astron. Italiana 80:711, arXiv:0908.4496

Magic Z, Collet R, Asplund M, Trampedach R, Hayek W, Chiavassa A, Stein RF, Nordlund A (2013) The Stagger-grid: A grid of 3D stellar atmosphere models. I. Methods and general 
properties. A\&A 557:A26, DOI 10.1051/0004-6361/201321274, arXiv:1302.2621

Maiorca E, Magrini L, Busso M, Randich S, Palmerini S, Trippella O (2012) News on the s Process from Young Open Clusters. ApJ 747:53, DOI 10.1088/0004-637X/747/1/53, arXiv:1112.5290

Majewski SR, Schiavon RP, Frinchaboy PM, Allende Prieto C, Barkhouser R, Bizyaev D, Blank B, Brunner S, Burton A, Carrera R, et al (2017) The Apache Point Observatory Galactic Evolution Experiment (APOGEE). AJ 154:94, DOI 10.3847/1538-3881/aa784d, arXiv:1509.05420

Mashonkina L, Sitnova T, Belyaev AK (2017) Influence of inelastic collisions with hydrogen atoms on the non-LTE modelling of $\mathrm{CaI}$ and CaII lines in late-type stars. A\&A 605:A53, DOI 10.1051/0004-6361/201731236, arXiv:1707.04399

Mashonkina LI, Belyaev AK, Shi JR (2016) Influence of inelastic collisions with hydrogen atoms on the formation of AlI and SiI lines in stellar spectra. Astronomy Letters 42:366378, DOI 10.1134/S1063773716050078, arXiv:1605.02957

Matteucci F, Brocato E (1990) Metallicity distribution and abundance ratios in the stars of the Galactic bulge. ApJ 365:539-543, DOI 10.1086/169508

Matteucci F, Greggio L (1986) Relative roles of type I and II supernovae in the chemical enrichment of the interstellar gas. A\&A 154:279-287

Matteucci F, Spitoni E, Recchi S, Valiante R (2009) The effect of different type Ia supernova progenitors on Galactic chemical evolution. A\&A 501:531-538, DOI 10.1051/0004-6361/ 200911869, arXiv:0905.0272

McWilliam A (2016) The Chemical Composition of the Galactic Bulge and Implications for its Evolution. PASA 33:e040, DOI 10.1017/pasa.2016.32, arXiv:1607.05299

McWilliam A, Rich RM (1994) The first detailed abundance analysis of Galactic bulge K giants in Baade's window. ApJS 91:749-791, DOI 10.1086/191954

McWilliam A, Preston GW, Sneden C, Searle L (1995) Spectroscopic Analysis of 33 of the Most Metal Poor Stars. II. AJ 109:2757, DOI 10.1086/117486

McWilliam A, Wallerstein G, Mottini M (2013) Chemistry of the Sagittarius Dwarf Galaxy: A Top-light Initial Mass Function, Outflows, and the R-process. ApJ 778:149, DOI 10.1088/ 0004-637X/778/2/149, arXiv: 1309.2974

Melendez J, Ramirez I (2016) Planet signatures in the chemical composition of Sun-like stars. ArXiv e-prints arXiv: 1611.04064

Meléndez J, Asplund M, Gustafsson B, Yong D (2009) The Peculiar Solar Composition and Its Possible Relation to Planet Formation. ApJL 704:L66-L70, DOI 10.1088/0004-637X/ 704/1/L66, arXiv:0909.2299

Milone AP, Marino AF, Piotto G, Bedin LR, Anderson J, Aparicio A, Bellini A, Cassisi S, D'Antona F, Grundahl F, Monelli M, Yong D (2013) A WFC3/HST View of the Three Stellar Populations in the Globular Cluster NGC 6752. ApJ 767:120, DOI 10.1088/0004-637X/767/2/120, arXiv:1301.7044

Minchev I, Chiappini C, Martig M (2014) Chemodynamical evolution of the Milky Way disk. II. Variations with Galactic radius and height above the disk plane. A\&A 572:A92, DOI 10.1051/0004-6361/201423487, arXiv:1401.5796

Mitschang AW, De Silva G, Zucker DB, Anguiano B, Bensby T, Feltzing S (2014) Quantitative chemical tagging, stellar ages and the chemo-dynamical evolution of the Galactic disc. MNRAS 438:2753-2764, DOI 10.1093/mnras/stt2320, arXiv:1312.1759

Moore CS, Uitenbroek H, Rempel M, Criscuoli S, Rast MP (2015) The Effects of Magnetic Field Morphology on the Determination of Oxygen and Iron Abundances in the Solar Photosphere. ApJ 799:150, DOI 10.1088/0004-637X/799/2/150

Morel T (2018) The chemical composition of $\alpha$ Centauri AB revisited. A\&A 615:A172, DOI 10.1051/0004-6361/201833125, arXiv: 1805.00929

Nandakumar G, Ryde N, Schultheis M, Thorsbro B, Jönsson H, Barklem PS, Rich RM, Fragkoudi F (2018) Chemical Characterization of the Inner Galactic bulge: North-South Symmetry. MNRAS DOI 10.1093/mnras/sty1255, arXiv:1805.05037

Ness M, Hogg DW, Rix HW, Ho AYQ, Zasowski G (2015) The Cannon: A data-driven approach to Stellar Label Determination. ApJ 808:16, DOI 10.1088/0004-637X/808/1/16, arXiv:1501.07604

Ness M, Rix HW, Hogg DW, Casey AR, Holtzman J, Fouesneau M, Zasowski G, Geisler D, Shetrone M, Minniti D, et al (2018) Galactic Doppelgängers: The Chemical Similarity Among Field Stars and Among Stars with a Common Birth Origin. ApJ 853:198, DOI 10.3847/1538-4357/aa9d8e, arXiv:1701.07829 
Nieva MF, Przybilla N (2012) Present-day cosmic abundances. A comprehensive study of nearby early B-type stars and implications for stellar and Galactic evolution and interstellar dust models. A\&A 539:A143, DOI 10.1051/0004-6361/201118158, arXiv:1203.5787

Nissen PE (2013) The carbon-to-oxygen ratio in stars with planets. A\&A 552:A73, DOI 10.1051/0004-6361/201321234, arXiv:1303.1726

Nissen PE (2015) High-precision abundances of elements in solar twin stars. Trends with stellar age and elemental condensation temperature. A\&A 579:A52, DOI 10.1051/0004-6361/ 201526269, arXiv:1504.07598

Nissen PE (2016) High-precision abundances of Sc, Mn, Cu, and Ba in solar twins. Trends of element ratios with stellar age. A\&A 593:A65, DOI 10.1051/0004-6361/201628888, arXiv: 1606.08399

Nissen PE, Schuster WJ (1997) Chemical composition of halo and disk stars with overlapping metallicities. A\&A 326:751-762

Nissen PE, Schuster WJ (2010) Two distinct halo populations in the solar neighborhood. Evidence from stellar abundance ratios and kinematics. A\&A 511:L10, DOI 10.1051/ 0004-6361/200913877, arXiv:1002.4514

Nissen PE, Schuster WJ (2011) Two distinct halo populations in the solar neighborhood. II. Evidence from stellar abundances of $\mathrm{Mn}, \mathrm{Cu}, \mathrm{Zn}, \mathrm{Y}$, and Ba. A\&A 530:A15, DOI 10.1051/0004-6361/201116619, arXiv:1103.4755

Nissen PE, Chen YQ, Carigi L, Schuster WJ, Zhao G (2014) Carbon and oxygen abundances in stellar populations. A\&A 568:A25, DOI 10.1051/0004-6361/201424184, arXiv:1406.5218

Nissen PE, Silva Aguirre V, Christensen-Dalsgaard J, Collet R, Grundahl F, Slumstrup D (2017) High-precision abundances of elements in Kepler LEGACY stars. Verification of trends with stellar age. A\&A 608:A112, DOI 10.1051/0004-6361/201731845, arXiv: 1710.03544

Nomoto K, Kobayashi C, Tominaga N (2013) Nucleosynthesis in Stars and the Chemical Enrichment of Galaxies. ARA\&A 51:457-509, DOI 10.1146/annurev-astro-082812-140956

Nordlander T, Lind K (2017) Non-LTE aluminium abundances in late-type stars. A\&A 607:A75, DOI 10.1051/0004-6361/201730427, arXiv:1708.01949

Nordlander T, Korn AJ, Richard O, Lind K (2012) Atomic Diffusion and Mixing in Old Stars. III. Analysis of NGC 6397 Stars under New Constraints. ApJ 753:48, DOI 10.1088/ 0004-637X/753/1/48, arXiv: 1204.5600

Nordlund A, Dravins D (1990) Stellar granulation. III - Hydrodynamic model atmospheres. IV - Line formation in inhomogeneous stellar photospheres. V - Synthetic spectral lines in disk-integrated starlight. A\&A 228:155-217

Nordlund A, Stein RF (1990) 3-D simulations of solar and stellar convection and magnetoconvection. Computer Physics Communications 59:119-125, DOI 10.1016/0010-4655(90) 90161-S

Nordström B, Mayor M, Andersen J, Holmberg J, Pont F, Jørgensen BR, Olsen EH, Udry S, Mowlavi N (2004) The Geneva-Copenhagen survey of the Solar neighbourhood. Ages, metallicities, and kinematic properties of $\sim 14000 \mathrm{~F}$ and $\mathrm{G}$ dwarfs. A\&A 418:989-1019, DOI 10.1051/0004-6361:20035959, astro-ph/0405198

Oh S, Price-Whelan AM, Brewer JM, Hogg DW, Spergel DN, Myles J (2018) Kronos and Krios: Evidence for Accretion of a Massive, Rocky Planetary System in a Comoving Pair of Solar-type Stars. ApJ 854:138, DOI 10.3847/1538-4357/aaab4d, arXiv:1709.05344

Olsen EH (1988) The intrinsic colour calibration of Stromgren photometry for F-type stars. A\&A 189:173-178

O'Malley EM, McWilliam A, Chaboyer B, Thompson I (2017) A Differential Abundance Analysis of Very Metal-poor Stars. ApJ 838:90, DOI 10.3847/1538-4357/aa62a2, arXiv: 1703.00019

Önehag A, Korn A, Gustafsson B, Stempels E, Vandenberg DA (2011) M67-1194, an unusually Sun-like solar twin in M67. A\&A 528:A85, DOI 10.1051/0004-6361/201015138, arXiv: 1009.4579

Önehag A, Heiter U, Gustafsson B, Piskunov N, Plez B, Reiners A (2012) M-dwarf metallicities. A high-resolution spectroscopic study in the near infrared. A\&A 542:A33, DOI 10.1051/ 0004-6361/201118101, arXiv:1112.0141

Önehag A, Gustafsson B, Korn A (2014) Abundances and possible diffusion of elements in M 67 stars. A\&A 562:A102, DOI 10.1051/0004-6361/201322663, arXiv:1310.6297

Payne CH (1928) On the Contours of Stellar Absorption Lines, and the Composition of Stellar Atmospheres. Proceedings of the National Academy of Science 14:399-406, DOI 10.1073/ 
pnas.14.5.399

Payne-Gaposchkin C, Whipple FL (1940) Synthetic Spectra for Supernovae. Proceedings of the National Academy of Science 26:264-272, DOI 10.1073/pnas.26.4.264

Pereira TMD, Asplund M, Kiselman D (2009) Oxygen lines in solar granulation. II. Centre-to-limb variation, NLTE line formation, blends, and the solar oxygen abundance. A\&A 508:1403-1416, DOI 10.1051/0004-6361/200912840, arXiv:0909.2310

Pereira TMD, Asplund M, Collet R, Thaler I, Trampedach R, Leenaarts J (2013) How realistic are solar model atmospheres? A\&A 554:A118, DOI 10.1051/0004-6361/201321227, arXiv:1304.4932

Perets HB, Gal-Yam A, Mazzali PA, Arnett D, Kagan D, Filippenko AV, Li W, Arcavi I, Cenko SB, Fox DB, et al (2010) A faint type of supernova from a white dwarf with a helium-rich companion. Nature 465:322-325, DOI 10.1038/nature09056, arXiv:0906.2003

Peterson RC (1980) Evidence from sodium-abundance variations among red giants of M13 for inhomogeneities in the protocluster gas. ApJL 237:L87-L91, DOI 10.1086/157950

Petigura EA, Marcy GW (2011) Carbon and Oxygen in Nearby Stars: Keys to Protoplanetary Disk Chemistry. ApJ 735:41, DOI 10.1088/0004-637X/735/1/41, arXiv:1106.5449

Pignatari M, Gallino R, Heil M, Wiescher M, Käppeler F, Herwig F, Bisterzo S (2010) The Weak s-Process in Massive Stars and its Dependence on the Neutron Capture Cross Sections. ApJ 710:1557-1577, DOI 10.1088/0004-637X/710/2/1557

Pinsonneault MH, Elsworth Y, Epstein C, Hekker S, Mészáros S, Chaplin WJ, Johnson JA, García RA, Holtzman J, Mathur S, et al (2014) The APOKASC Catalog: An Asteroseismic and Spectroscopic Joint Survey of Targets in the Kepler Fields. ApJS 215:19, DOI 10.1088/ 0067-0049/215/2/19, arXiv:1410.2503

Piskunov N, Valenti JA (2017) Spectroscopy Made Easy: Evolution. A\&A 597:A16, DOI 10.1051/0004-6361/201629124, arXiv:1606.06073

Prantzos N, de Laverny P, Guiglion G, Recio-Blanco A, Worley CC (2017) The AMBRE project: a study of Li evolution in the Galactic thin and thick discs. A\&A 606:A132, DOI 10.1051/0004-6361/201731188, arXiv:1709.03998

Ramírez I, Meléndez J, Cornejo D, Roederer IU, Fish JR (2011) Elemental Abundance Differences in the 16 Cygni Binary System: A Signature of Gas Giant Planet Formation? ApJ 740:76, DOI 10.1088/0004-637X/740/2/76, arXiv:1107.5814

Ramírez I, Meléndez J, Chanamé J (2012) Oxygen Abundances in Low- and High- $\alpha$ Field Halo Stars and the Discovery of Two Field Stars Born in Globular Clusters. ApJ 757:164, DOI 10.1088/0004-637X/757/2/164, arXiv:1208.3675

Ramírez I, Allende Prieto C, Lambert DL (2013) Oxygen Abundances in Nearby FGK Stars and the Galactic Chemical Evolution of the Local Disk and Halo. ApJ 764:78, DOI 10. 1088/0004-637X/764/1/78, arXiv:1301.1582

Ramírez I, Meléndez J, Bean J, Asplund M, Bedell M, Monroe T, Casagrande L, Schirbel L, Dreizler S, Teske J, Tucci Maia M, Alves-Brito A, Baumann P (2014) The Solar Twin Planet Search. I. Fundamental parameters of the stellar sample. A\&A 572:A48, DOI 10.1051/0004-6361/201424244, arXiv:1408.4130

Ramírez I, Khanal S, Aleo P, Sobotka A, Liu F, Casagrande L, Meléndez J, Yong D, Lambert DL, Asplund M (2015) The Dissimilar Chemical Composition of the Planethosting Stars of the XO-2 Binary System. ApJ 808:13, DOI 10.1088/0004-637X/808/1/13, arXiv:1506.01025

Recio-Blanco A, Rojas-Arriagada A, de Laverny P, Mikolaitis S, Hill V, Zoccali M, FernándezTrincado JG, Robin AC, Babusiaux C, Gilmore G, Randich S, et al (2017) The Gaia-ESO Survey: Low- $\alpha$ element stars in the Galactic bulge. A\&A 602:L14, DOI 10.1051/0004-6361/ 201630220, arXiv:1702.04500

Reddy ABS, Lambert DL (2015) Local associations and the barium puzzle. MNRAS 454:19761991, DOI 10.1093/mnras/stv1876, arXiv:1508.02815

Reddy ABS, Lambert DL (2017) Solar Twins and the Barium Puzzle. ApJ 845:151, DOI 10.3847/1538-4357/aa81d6, arXiv:1707.07051

Reddy BE, Lambert DL, Allende Prieto C (2006) Elemental abundance survey of the Galactic thick disc. MNRAS 367:1329-1366, DOI 10.1111/j.1365-2966.2006.10148.x, astro-ph/0512505

Reggiani H, Meléndez J, Yong D, Ramírez I, Asplund M (2016) First high-precision differential abundance analysis of extremely metal-poor stars. A\&A 586:A67, DOI 10.1051/ 0004-6361/201527439, arXiv:1512.03349

Reggiani H, Meléndez J, Kobayashi C, Karakas A, Placco V (2017) Constraining cosmic scatter 
in the Galactic halo through a differential analysis of metal-poor stars. A\&A 608:A46, DOI 10.1051/0004-6361/201730750, arXiv:1709.03750

Rojas-Arriagada A, Recio-Blanco A, de Laverny P, Mikolaitis Š, Matteucci F, Spitoni E, Schultheis M, Hayden M, Hill V, Zoccali M, et al (2017) The Gaia-ESO Survey: Exploring the complex nature and origins of the Galactic bulge populations. A\&A 601:A140, DOI 10.1051/0004-6361/201629160, arXiv:1704.03325

Romano D, Matteucci F (2007) Contrasting copper evolution in $\omega$ Centauri and the Milky Way. MNRAS 378:L59-L63, DOI 10.1111/j.1745-3933.2007.00320.x, astro-ph/0703760

Russell HN (1929) On the Composition of the Sun's Atmosphere. ApJ 70:11, DOI 10.1086/ 143197

Ryde N, Schultheis M, Grieco V, Matteucci F, Rich RM, Uttenthaler S (2016) Chemical Evolution of the Inner 2 Degrees of the Milky Way Bulge: $[\alpha / \mathrm{Fe}]$ Trends and Metallicity Gradients. AJ 151:1, DOI 10.3847/0004-6256/151/1/1, arXiv:1510.02622

Saffe C, Jofré E, Martioli E, Flores M, Petrucci R, Jaque Arancibia M (2017) Signatures of rocky planet engulfment in HAT-P-4. Implications for chemical tagging studies. A\&A 604:L4, DOI 10.1051/0004-6361/201731430, arXiv:1707.02180

Santos NC, Israelian G, Mayor M (2001) The metal-rich nature of stars with planets. A\&A 373:1019-1031, DOI 10.1051/0004-6361:20010648, astro-ph/0105216

Schultheis M, Rojas-Arriagada A, García Pérez AE, Jönsson H, Hayden M, Nandakumar G, Cunha K, Allende Prieto C, Holtzman JA, Beers TC, et al (2017) Baade's window and APOGEE. Metallicities, ages, and chemical abundances. A\&A 600:A14, DOI 10.1051/ 0004-6361/201630154, 1702.01547

Schuster WJ, Moitinho A, Márquez A, Parrao L, Covarrubias E (2006) uvby- $\beta$ photometry of high-velocity and metal-poor stars. XI. Ages of halo and old disk stars. A\&A 445:939-958, DOI 10.1051/0004-6361:20053796, astro-ph/0510313

Scott P, Grevesse N, Asplund M, Sauval AJ, Lind K, Takeda Y, Collet R, Trampedach R, Hayek W (2015) The elemental composition of the Sun. I. The intermediate mass elements $\mathrm{Na}$ to Ca. A\&A 573:A25, DOI 10.1051/0004-6361/201424109, arXiv:1405.0279

Searle L, Zinn R (1978) Compositions of halo clusters and the formation of the galactic halo. ApJ 225:357-379, DOI 10.1086/156499

Shchukina N, Trujillo Bueno J (2015) The impact of surface dynamo magnetic fields on the solar iron abundance. A\&A 579:A112, DOI 10.1051/0004-6361/201425569

Shchukina N, Sukhorukov A, Trujillo Bueno J (2016) Impact of surface dynamo magnetic fields on the solar abundance of the CNO elements. A\&A 586:A145, DOI 10.1051/0004-6361/ 201526452

Shetrone M, Venn KA, Tolstoy E, Primas F, Hill V, Kaufer A (2003) VLT/UVES Abundances in Four Nearby Dwarf Spheroidal Galaxies. I. Nucleosynthesis and Abundance Ratios. AJ 125:684-706, DOI 10.1086/345966, astro-ph/0211167

Shetrone M, Bizyaev D, Lawler JE, Allende Prieto C, Johnson JA, Smith VV, Cunha K, Holtzman J, García Pérez AE, Mészáros S, et al (2015) The SDSS-III APOGEE Spectral Line List for H-band Spectroscopy. ApJS 221:24, DOI 10.1088/0067-0049/221/2/24, arXiv: 1502.04080

Shetrone MD, Côté P, Sargent WLW (2001) Abundance Patterns in the Draco, Sextans, and Ursa Minor Dwarf Spheroidal Galaxies. ApJ 548:592-608, DOI 10.1086/319022, astro-ph/0009505

Shi JR, Yan HL, Zhou ZM, Zhao G (2018) NLTE Analysis of Copper Lines in Different Stellar Populations. ApJ 862:71, DOI 10.3847/1538-4357/aacb22, arXiv:1806.02225

Silva Aguirre V, Davies GR, Basu S, Christensen-Dalsgaard J, Creevey O, Metcalfe TS, Bedding TR, Casagrande L, Handberg R, Lund MN, Nissen PE, et al (2015) Ages and fundamental properties of Kepler exoplanet host stars from asteroseismology. MNRAS 452:21272148, DOI 10.1093/mnras/stv1388, arXiv:1504.07992

Silva Aguirre V, Lund MN, Antia HM, Ball WH, Basu S, Christensen-Dalsgaard J, Lebreton Y, Reese DR, Verma K, Casagrande L, et al (2017) Standing on the Shoulders of Dwarfs: the Kepler Asteroseismic LEGACY Sample. II.Radii, Masses, and Ages. ApJ 835:173, DOI 10.3847/1538-4357/835/2/173, arXiv:1611.08776

Skúladóttir Á, Tolstoy E, Salvadori S, Hill V, Pettini M (2017) Zinc abundances in the Sculptor dwarf spheroidal galaxy. A\&A 606:A71, DOI 10.1051/0004-6361/201731158, arXiv: 1708.00511

Smiljanic R, Pasquini L, Charbonnel C, Lagarde N (2010) Beryllium abundances along the evolutionary sequence of the open cluster IC 4651. In: Charbonnel C, Tosi M, Primas F, 
Chiappini C (eds) Light Elements in the Universe, IAU Symposium, vol 268, pp 357-358, DOI 10.1017/S174392131000445X, arXiv: 1001.1832

Smith VV, Lambert DL (1985) The chemical composition of red giants. I - Dredge-up in the M and MS stars. ApJ 294:326-338, DOI 10.1086/163300

Snaith ON, Haywood M, Di Matteo P, Lehnert MD, Combes F, Katz D, Gómez A (2014) The Dominant Epoch of Star Formation in the Milky Way Formed the Thick Disk. ApJL 781:L31, DOI 10.1088/2041-8205/781/2/L31, arXiv:1401.1835

Sneden C, Cowan JJ, Kobayashi C, Pignatari M, Lawler JE, Den Hartog EA, Wood MP (2016) Iron-group Abundances in the Metal-poor Main-Sequence Turnoff Star HD 84937. ApJ 817:53, DOI 10.3847/0004-637X/817/1/53, arXiv:1511.05985

Sousa SG, Santos NC, Adibekyan V, Delgado-Mena E, Israelian G (2015) ARES v2: new features and improved performance. A\&A 577:A67, DOI 10.1051/0004-6361/201425463, arXiv:1504.02725

Spina L, Meléndez J, Karakas AI, Ramírez I, Monroe TR, Asplund M, Yong D (2016a) Nucleosynthetic history of elements in the Galactic disk. [X/Fe]-age relations from high-precision spectroscopy. A\&A 593:A125, DOI 10.1051/0004-6361/201628557, arXiv:1606.04842

Spina L, Meléndez J, Ramírez I (2016b) Planet signatures and effect of the chemical evolution of the Galactic thin-disk stars. A\&A 585:A152, DOI 10.1051/0004-6361/201527429, arXiv: 1511.01012

Spina L, Meléndez J, Casey AR, Karakas AI, Tucci-Maia M (2018a) Chemical Inhomogeneities in the Pleiades: Signatures of Rocky-forming Material in Stellar Atmospheres. ApJ 863:179, DOI 10.3847/1538-4357/aad190, arXiv:1807.00941

Spina L, Meléndez J, Karakas AI, dos Santos L, Bedell M, Asplund M, Ramírez I, Yong D, Alves-Brito A, Bean JL, Dreizler S (2018b) The temporal evolution of neutron-capture elements in the Galactic discs. MNRAS 474:2580-2593, DOI 10.1093/mnras/stx2938, arXiv: 1711.03643

Spinrad H, Vardya MS (1966) Approximate Abundances of the Light Elements from the Molecular Spectra of M and S Stars. ApJ 146:399, DOI 10.1086/148904

Steffen M, Prakapavičius D, Caffau E, Ludwig HG, Bonifacio P, Cayrel R, Kučinskas A, Livingston WC (2015) The photospheric solar oxygen project. IV. 3D-NLTE investigation of the $777 \mathrm{~nm}$ triplet lines. A\&A 583:A57, DOI 10.1051/0004-6361/201526406, arXiv: 1508.03487

Stein RF, Nordlund $\AA$ (1998) Simulations of Solar Granulation. I. General Properties. ApJ 499:914-933, DOI 10.1086/305678

Stephens A, Boesgaard AM (2002) Abundances from High-Resolution Spectra of Kinematically Interesting Halo Stars. AJ 123:1647-1700, DOI 10.1086/338898

Stetson PB, Pancino E (2008) DAOSPEC: An Automatic Code for Measuring Equivalent Widths in High-Resolution Stellar Spectra. PASP 120:1332, DOI 10.1086/596126, arXiv:0811.2932

Strömgren B (1940) On the Chemical Composition of the Solar Atmosphere. In: Festschrift für Elis Strömgren, Munksgaard, E., p 218

Strömgren B, Gustafsson B, Olsen EH (1982) Evidence of helium abundance differences between the Hyades stars and field stars, and between Hyades stars and Coma cluster stars. PASP 94:5-15, DOI 10.1086/130932

Suárez-Andrés L, Israelian G, González Hernández JI, Adibekyan VZ, Delgado Mena E, Santos NC, Sousa SG (2018) C/O vs. Mg/Si ratios in solar type stars: The HARPS sample. A\&A 614:A84, DOI 10.1051/0004-6361/201730743, arXiv:1801.09474

Takeda Y, Tajitsu A (2015) Spectroscopic study of red giants in the Kepler field with asteroseismologically established evolutionary status and stellar parameters. MNRAS 450:397-413, DOI 10.1093/mnras/stv682, arXiv:1503.07595

Takeda Y, Hashimoto O, Taguchi H, Yoshioka K, Takada-Hidai M, Saito Y, Honda S (2005) Non-LTE Line-Formation and Abundances of Sulfur and Zinc in F, G, and K Stars. PASJ 57:751-768, DOI 10.1093/pasj/57.5.751, astro-ph/0509239

Teske JK, Ghezzi L, Cunha K, Smith VV, Schuler SC, Bergemann M (2015) Abundance Differences between Exoplanet Binary Host Stars XO-2N and XO-2S - Dependence on Stellar Parameters. ApJL 801:L10, DOI 10.1088/2041-8205/801/1/L10, arXiv:1501.02167

Teske JK, Khanal S, Ramírez I (2016) The Curious Case of Elemental Abundance Differences in the Dual Hot Jupiter Hosts WASP-94A and B. ApJ 819:19, DOI 10.3847/0004-637X/ 819/1/19, arXiv:1601.01731

Thiabaud A, Marboeuf U, Alibert Y, Leya I, Mezger K (2015) Elemental ratios in stars vs 
planets. A\&A 580:A30, DOI 10.1051/0004-6361/201525963, arXiv:1507.01343

Thomson W (1889) Constitution of Matter. In: Popular Lectures and Addresses, Macmillan \& Company, London \& New York, vol 1, p 73

Thorsbro B, Ryde N, Schultheis M, Hartman H, Rich RM, Lomaeva M, Origlia L, Jönsson H (2018) Evidence against anomalous compositions for giants in the Galactic Nuclear Star Cluster. ArXiv e-prints arXiv:1808.07489

Ting YS, Conroy C, Goodman A (2015) Prospects for Chemically Tagging Stars in the Galaxy. ApJ 807:104, DOI 10.1088/0004-637X/807/1/104, arXiv:1504.03327

Ting YS, Conroy C, Rix HW, Cargile P (2017) Prospects for Measuring Abundances of $>20$ Elements with Low-resolution Stellar Spectra. ApJ 843:32, DOI 10.3847/1538-4357/aa7688, arXiv: 1706.00111

Tinsley BM (1979) Stellar lifetimes and abundance ratios in chemical evolution. ApJ 229:10461056, DOI $10.1086 / 157039$

Tolstoy E, Hill V, Tosi M (2009) Star-Formation Histories, Abundances, and Kinematics of Dwarf Galaxies in the Local Group. ARA\&A 47:371-425, DOI 10.1146/ annurev-astro-082708-101650, arXiv:0904.4505

Trampedach R, Asplund M, Collet R, Nordlund Å, Stein RF (2013) A Grid of Threedimensional Stellar Atmosphere Models of Solar Metallicity. I. General Properties, Granulation, and Atmospheric Expansion. ApJ 769:18, DOI 10.1088/0004-637X/769/1/18, arXiv: 1303.1780

Tsuji T (1985) C, N, O, and their isotope abundances in coolest stars of the red giant branch. In: Jaschek M, Keenan PC (eds) Cool Stars with Excesses of Heavy Elements, Astrophysics and Space Science Library, vol 114, pp 295-298, DOI 10.1007/978-94-009-5325-3-33

Tucci Maia M, Meléndez J, Ramírez I (2014) High Precision Abundances in the 16 Cyg Binary System: A Signature of the Rocky Core in the Giant Planet. ApJL 790:L25, DOI 10.1088/ 2041-8205/790/2/L25, arXiv:1407.4132

Turcotte S, Richer J, Michaud G, Iglesias CA, Rogers FJ (1998) Consistent Solar Evolution Model Including Diffusion and Radiative Acceleration Effects. ApJ 504:539-558, DOI $10.1086 / 306055$

Unsöld A (1938) Physik der sternamospharen, MIT besonderer berucksichtigung der sonne. Berlin, Verlag von Julius Springer

Unterborn CT, Kabbes JE, Pigott JS, Reaman DM, Panero WR (2014) The Role of Carbon in Extrasolar Planetary Geodynamics and Habitability. ApJ 793:124, DOI 10.1088/ 0004-637X/793/2/124, arXiv: 1311.0024

Valenti JA, Piskunov N (1996) Spectroscopy made easy: A new tool for fitting observations with synthetic spectra. A\&AS 118:595-603

Van der Swaelmen M, Hill V, Primas F, Cole AA (2013) Chemical abundances in LMC stellar populations. II. The bar sample. A\&A 560:A44, DOI 10.1051/0004-6361/201321109, arXiv: 1306.4224

van Leeuwen F (2007) Validation of the new Hipparcos reduction. A\&A 474:653-664, DOI 10.1051/0004-6361:20078357, arXiv:0708.1752

Veyette MJ, Muirhead PS, Mann AW, Brewer JM, Allard F, Homeier D (2017) A Physically Motivated and Empirically Calibrated Method to Measure the Effective Temperature, Metallicity, and Ti Abundance of M Dwarfs. ApJ 851:26, DOI 10.3847/1538-4357/aa96aa, arXiv:1710.10259

Vieira T (1986) The chemical composition of Alpha Orionis. Uppsala Astronomical Observatory Reports 32

Vinyoles N, Serenelli AM, Villante FL, Basu S, Bergström J, Gonzalez-Garcia MC, Maltoni M, Peña-Garay C, Song N (2017) A New Generation of Standard Solar Models. ApJ 835:202, DOI $10.3847 / 1538-4357 / 835 / 2 / 202$, arXiv:1611.09867

Vitense E (1953) Die Wasserstoffkonvektionszone der Sonne. Mit 11 Textabbildungen. Zeitschrift für Astrophysik 32:135

Wallace L, Hinkle KH, Livingston WC, Davis SP (2011) An Optical and Near-infrared (2958$9250 \AA$ A) Solar Flux Atlas. ApJS 195:6, DOI 10.1088/0067-0049/195/1/6

Wallerstein G (1962) Abundances in G Dwarfs.VI. a Survey of Field Stars. ApJS 6:407, DOI $10.1086 / 190067$

Wedemeyer S, Freytag B, Steffen M, Ludwig HG, Holweger H (2004) Numerical simulation of the three-dimensional structure and dynamics of the non-magnetic solar chromosphere. A\&A 414:1121-1137, DOI 10.1051/0004-6361:20031682, astro-ph/0311273

Westin J, Sneden C, Gustafsson B, Cowan JJ (2000) The r-Process-enriched Low-Metallicity 
Giant HD 115444. ApJ 530:783-799, DOI 10.1086/308407, astro-ph/9910376

Worrall G (1972) Can Astrophysical Abundances be Taken Seriously? Nature 236:15-18, DOI $10.1038 / 236015 \mathrm{aO}$

Wyatt MC (2008) Evolution of Debris Disks. ARA\&A 46:339-383, DOI 10.1146/annurev.astro. 45.051806.110525

Yan HL, Shi JR, Nissen PE, Zhao G (2016) Non-LTE analysis of copper abundances for the two distinct halo populations in the solar neighborhood. A\&A 585:A102, DOI 10.1051/ 0004-6361/201527491, arXiv:1511.04168

Yong D, Carney BW, Friel ED (2012) Elemental Abundance Ratios in Stars of the Outer Galactic Disk. IV. A New Sample of Open Clusters. AJ 144:95, DOI 10.1088/0004-6256/ 144/4/95, arXiv:1206.6931

Yong D, Meléndez J, Grundahl F, Roederer IU, Norris JE, Milone AP, Marino AF, Coelho P, McArthur BE, Lind K, Collet R, Asplund M (2013) High precision differential abundance measurements in globular clusters: chemical inhomogeneities in NGC 6752 . MNRAS 434:3542-3565, DOI 10.1093/mnras/stt1276, arXiv:1307.4486

Zhao G, Zhao YH, Chu YQ, Jing YP, Deng LC (2012) LAMOST spectral survey - An overview. Research in Astronomy and Astrophysics 12:723-734, DOI 10.1088/1674-4527/12/7/002

Zolotov A, Willman B, Brooks AM, Governato F, Brook CB, Hogg DW, Quinn T, Stinson G (2009) The Dual Origin of Stellar Halos. ApJ 702:1058-1067, DOI 10.1088/0004-637X/ 702/2/1058, arXiv:0904.3333

Zolotov A, Willman B, Brooks AM, Governato F, Hogg DW, Shen S, Wadsley J (2010) The Dual Origin of Stellar Halos. II. Chemical Abundances as Tracers of Formation History. ApJ 721:738-743, DOI 10.1088/0004-637X/721/1/738, arXiv:1004.3789 